\title{
Neutrino Processes in Neutron Stars
}

\author{
Evgeni E. Kolomeitsev ${ }^{1, \mathrm{a}}$ and Dmitry N. Voskresensky ${ }^{2,3, b}$ \\ 1 Matej Bel University, SK-97401 Banska Bystrica, Slovakia \\ 2 GSI, D-64291 Darmstadt, Germany \\ 3 Moscow Engineering Physical Insitute, RUS-115409, Moscow, Russia
}

\begin{abstract}
The aim of these lectures is to introduce basic processes responsible for cooling of neutron stars and to show how to calculate the neutrino production rate in dense strongly interacting nuclear medium. The formalism is presented that treats on equal footing one-nucleon and multiple-nucleon processes and reactions with virtual bosonic modes and condensates. We demonstrate that neutrino emission from dense hadronic component in neutron stars is subject of strong modifications due to collective effects in the nuclear matter. With the most important in-medium processes incorporated in the cooling code an overall agreement with available soft $X$ ray data can be easily achieved. With these findings the so-called "standard" and "non-standard" cooling scenarios are replaced by one general "nuclear medium cooling scenario" which relates slow and rapid neutron star coolings to the star masses (interior densities).

The lectures are split in four parts.
\end{abstract}

Part I: After short introduction to the neutron star cooling problem we show how to calculate neutrino reaction rates of the most efficient one-nucleon and two-nucleon processes. No medium effects are taken into account in this instance. The effects of a possible nucleon pairing are discussed. We demonstrate that the data on neutron star cooling cannot be described without inclusion of medium effects. It motivates an assumption that masses of the neutron stars are different and that neutrino reaction rates should be strongly density dependent.

Part II: We introduce the Green's function diagram technique for systems in and out of equilibrium and the optical theorem formalism. The latter allows to perform calculations of production rates with full Green's functions including all off-mass-shell effects. We demonstrate how this formalism works within the quasiparticle approximation.

Part III: The basic concepts of the nuclear Fermi liquid approach are introduced. We show how strong interaction effects can be included within the Green's function formalism. Softening of the pion mode with an baryon density increase is explicitly incorporated. We show examples of inconsistencies in calculations without inclusion of medium effects. Then we demonstrate calculations of different reaction rates in non-superfluid nuclear matter with taking into account medium effects. Many new reaction channels are open up in the medium and should be analyzed.

Part IV: We discuss the neutrino production reactions in superfluid nuclear systems. The reaction rates of processes associated with the pair breaking and formation are calculated. Special attention is focused on the gauge invariance and the exact fulfillment of the Ward identities for the vector current. Finally we present comparison of calculations of neutron star cooling performed within nuclear medium cooling scenario with the available data.

\section{References}

1. S. Shapiro, S.A. Teukolsky, Black Holes, White Dwarfs and Neutron Stars: The Physics of Compact Objects ( Wiley, New York 1983).

2. D. Pines, R. Tamagaki, S. Tsuruta (eds.), Neutron Stars (Addison-Weseley, New York 1992).

3. M. A. Alpar, Ü. Kiziloglu, J. van Paradijs (eds.), The Lives of the Neutron Stars (NATO ASI Ser. C, 450; Dordrecht: Kluwer 1995).

\footnotetext{
a e-mail: kolomeitsev@fpv.umb.sk

b e-mail: D.Voskresensky@gsi.de
}

4. F. Weber, Pulsars as Astrophysical Laboratories for Nuclear and Particle Physics (IoP Publishing, 1999) $682 \mathrm{pp}$.

5. P. Haensel, A.Y. Potekhin, D.G. Yakovlev, Neutron Stars 1. Equation of State and Structure (Springer, 2007) $619 \mathrm{pp}$.

6. A.B. Migdal, Theory of Finite Fermi Systems and Properties of Atomic Nuclei (Willey and Sons, New York 1967; second. ed. (in Rus.), Nauka, Moscow, 1983).

This is an Open Access article distributed under the terms of the Creative Commons Attribution-Noncommercial License 3.0, which permits unrestricted use, distribution, and reproduction in any noncommercial medium, provided the original work is properly cited. 


\section{Neutrino Processes in Neutron Stars}
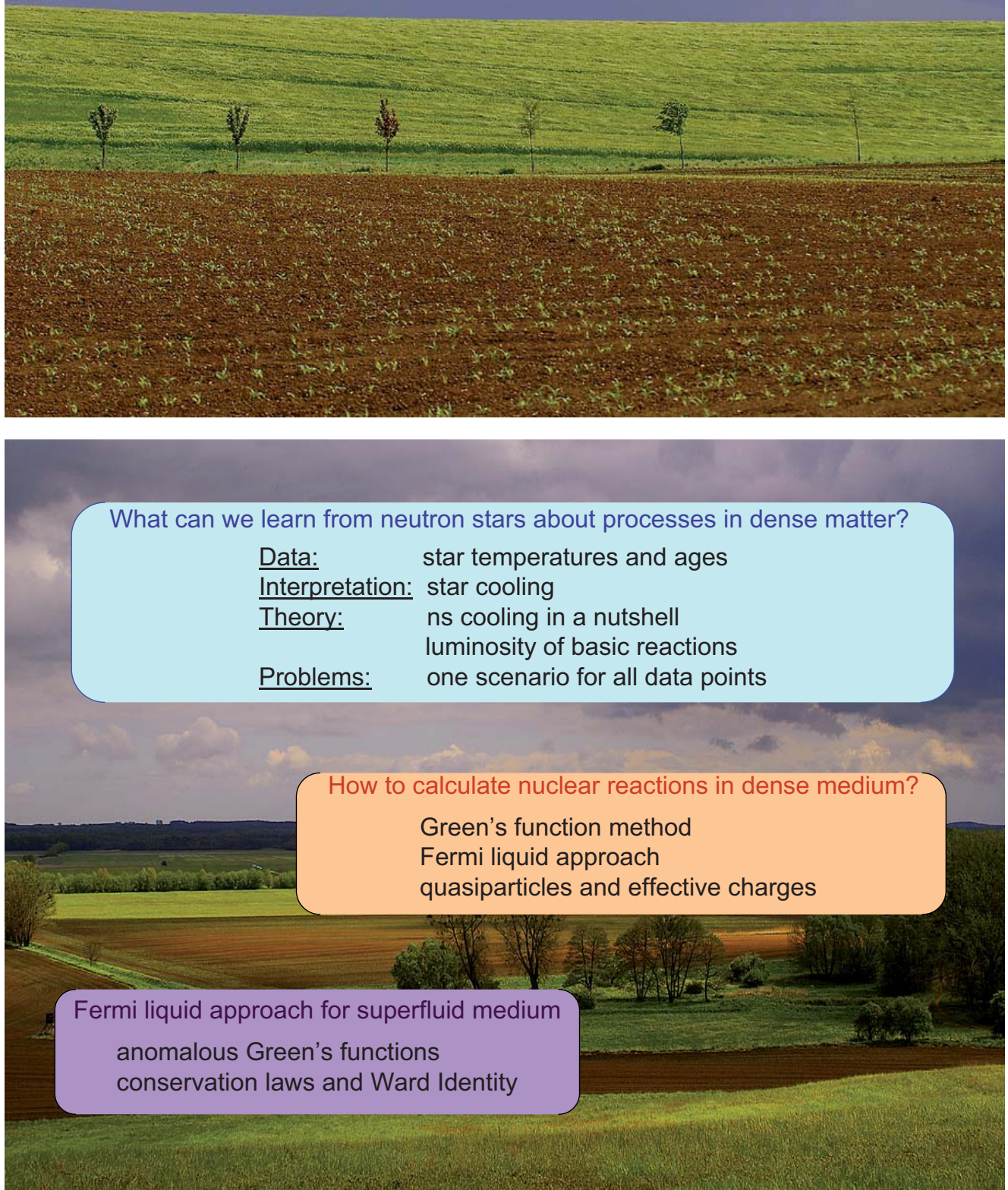


\section{Structure of NS}

- atmosphere $\mathrm{mm}-10 \mathrm{~cm}, \rho \lesssim 10^{6} \mathrm{~g} / \mathrm{cm}^{3}$,

plasma: determines photon radiation; $T_{\text {surf }}, B_{\text {surf }}$ affect EOS;

- outer crust $\sim 10^{2} \mathrm{~m}, \rho<\rho_{d}=4 \cdot 10^{11} \mathrm{~g} / \mathrm{cm}^{3}$, solid of heavy nuclei and relativistic electrons;

- inner crust $\sim \mathrm{km}, \rho \lesssim 0.5 \div 0.7 \rho_{0}$, neutronized nuclei + neutron gas + rel. electrons; $0.3 \rho_{0} \lesssim \rho \lesssim 0.5 \div 0.7 \rho_{0}$, nuclear pasta $=$ mixed phase: nuclear drops, rods, slabs, etc.:

- onter core $\gtrsim$ from several $\mathrm{km}$, maybe, up to the center, $\rho \lesssim 2 \div 4 \rho_{0}$, superfluid (at $T \lesssim \mathrm{MeV}$ ) of $n n, p p+$ normal electrons;

- inner core up to center (larger for massive NS),

Kingdom of Exotics:

possible mixed phases between npe, $\pi_{c}, K_{c}, H, q$-CSC(?), pure phases $\pi_{c}, K_{c}, H, q$-CSC?

\section{Neutrino probe}

At temperatures smaller than the opacity temperature $\left(T^{o p a c} \sim 1\right.$-few $\left.\mathrm{MeV}\right)$ mean free path of neutrinos and antineutrinos is larger than the neutron star radius

$$
\lambda_{\nu} \gg R \simeq 10 \mathrm{~km}
$$

\section{$\Rightarrow$ white body radiation problem}

After $>10^{5} \mathrm{yr}$-black body radiation of photons

At temperatures $T>T^{\text {opac }} \quad \lambda_{\nu}<R$

neutrino transport problem

important for supernova 


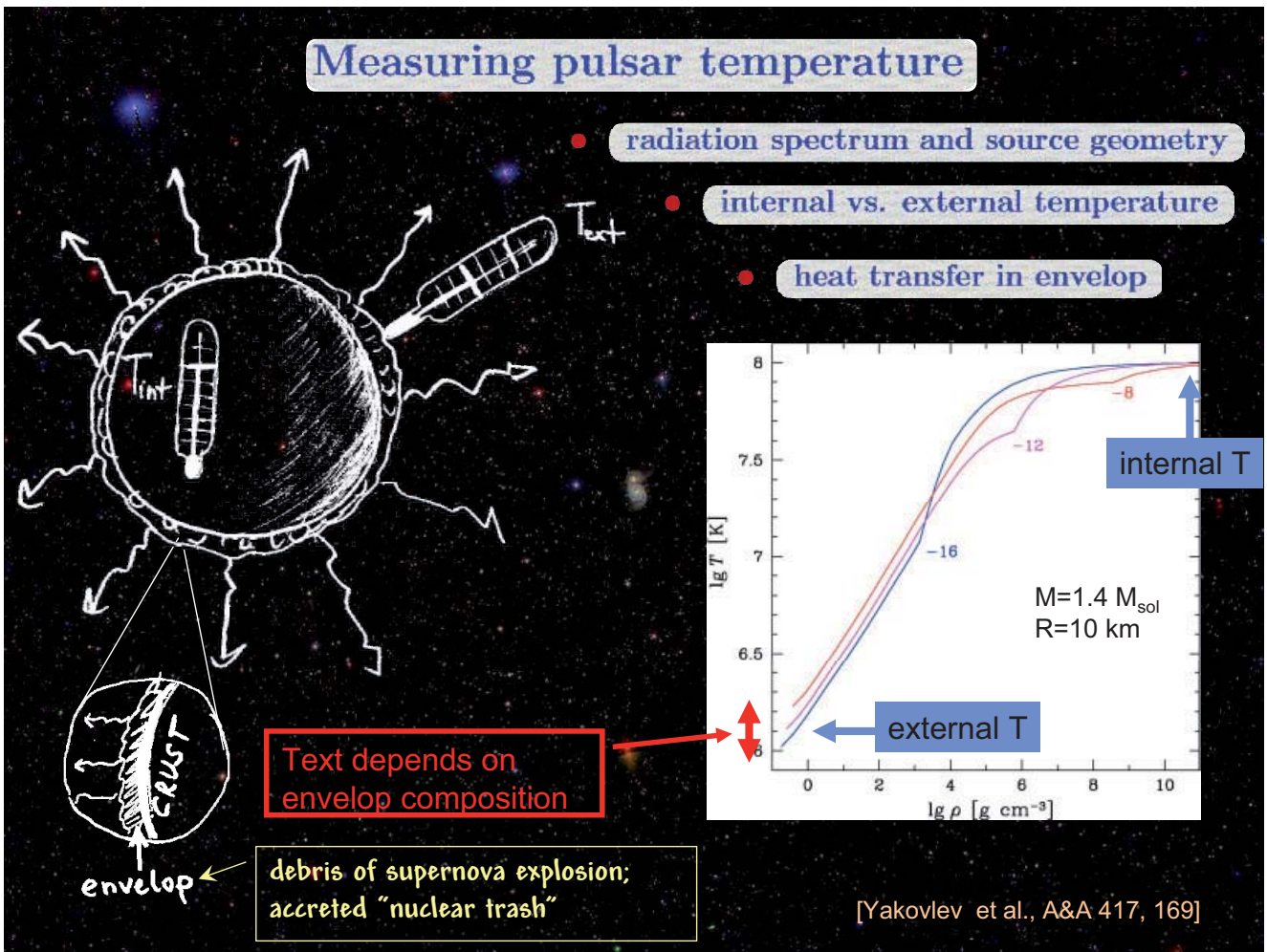

\section{Measuring pulsar age}

- pulsar spin-down rotation frequency $\Omega=2 \pi / P$ period

for non-accreting systems, period increases with time

power-law spin-down $\Omega=\frac{d \Omega}{d t}=-k \Omega^{n}$ braking index $n=\ddot{\Omega} \Omega / \dot{\Omega}^{2}$

for magnetic dipole spin-down $n=3$

$$
t=\frac{P}{(n-1) \dot{P}}\left[1-\left(\frac{P_{0}}{P}\right)^{n-1}\right]
$$

- kinematic age

"spin-down age"

1) age of the associated $S N R$

2) pulsar speed and position w.r. to the geometric center of the associated SNR
Crab : 1054 AD

Cassiopeia A: 1680 AD

Tycho's SN: 1572 AD 


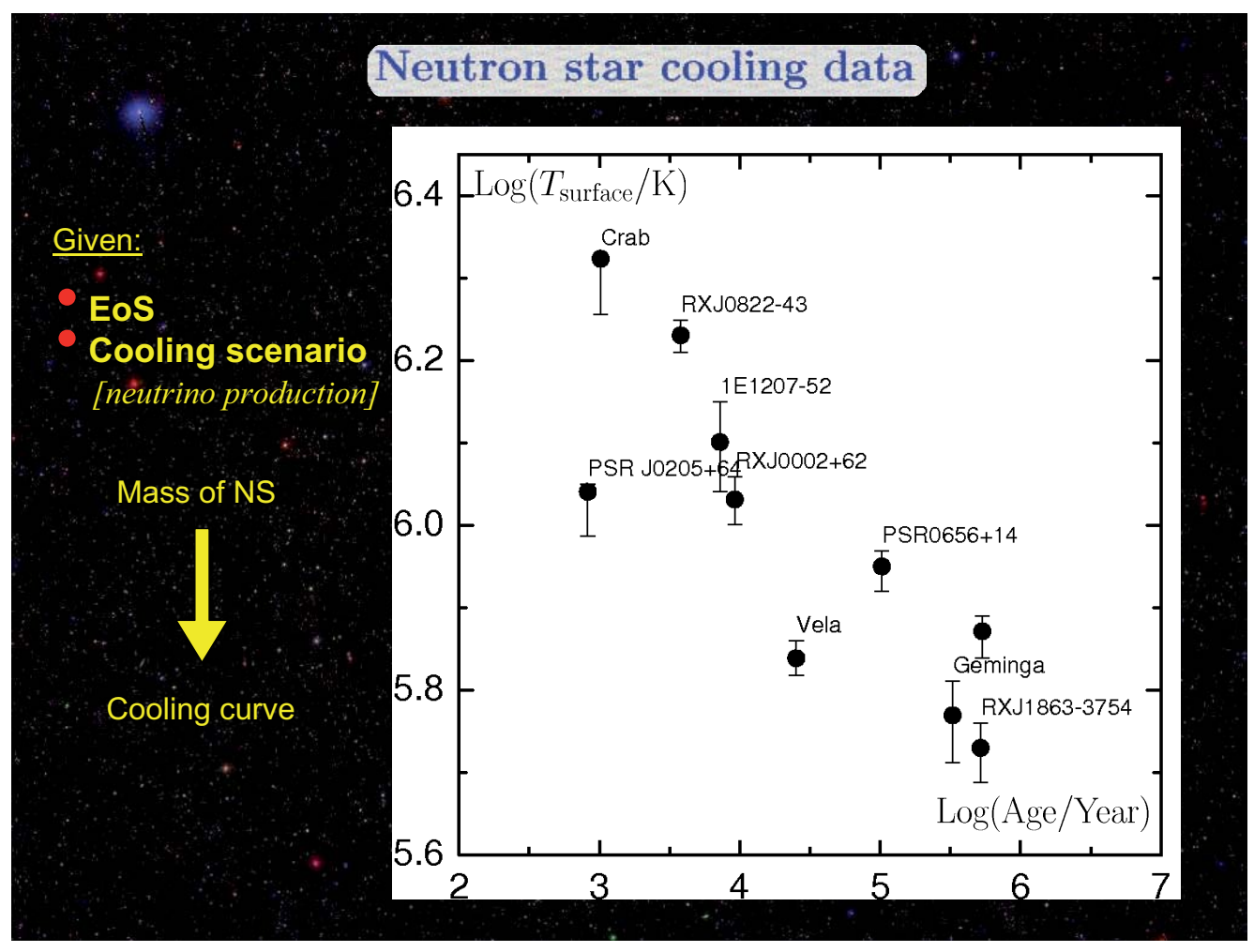

Neutron star cooling data

3 groups:

slow cooling

$$
\log \left(T_{\text {surface }} / \mathrm{K}\right)
$$

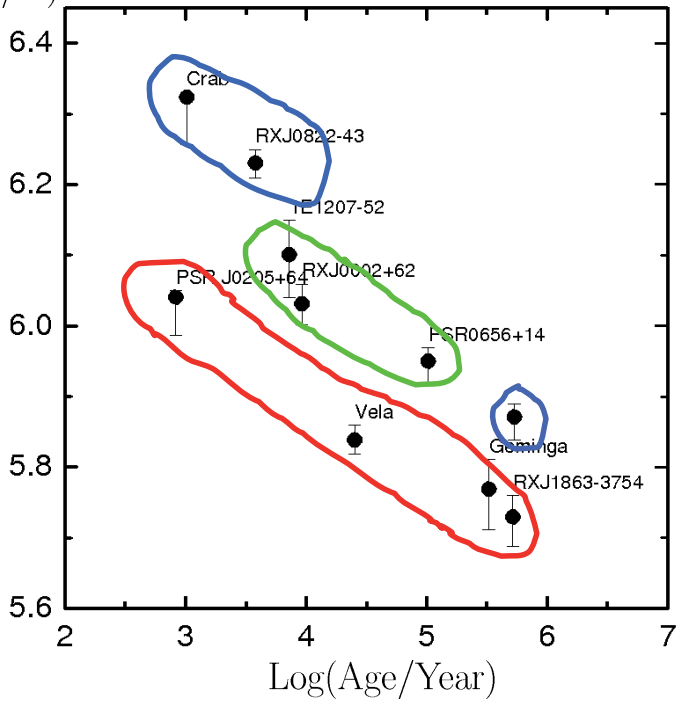

How to describe all groups within one cooling scenario? 


\section{Neutrino emission reactions}

$T<T_{\text {opac }} \sim 10^{-1} \div 10^{0} \mathrm{MeV} \quad$ neutron star is transparent for neutrino

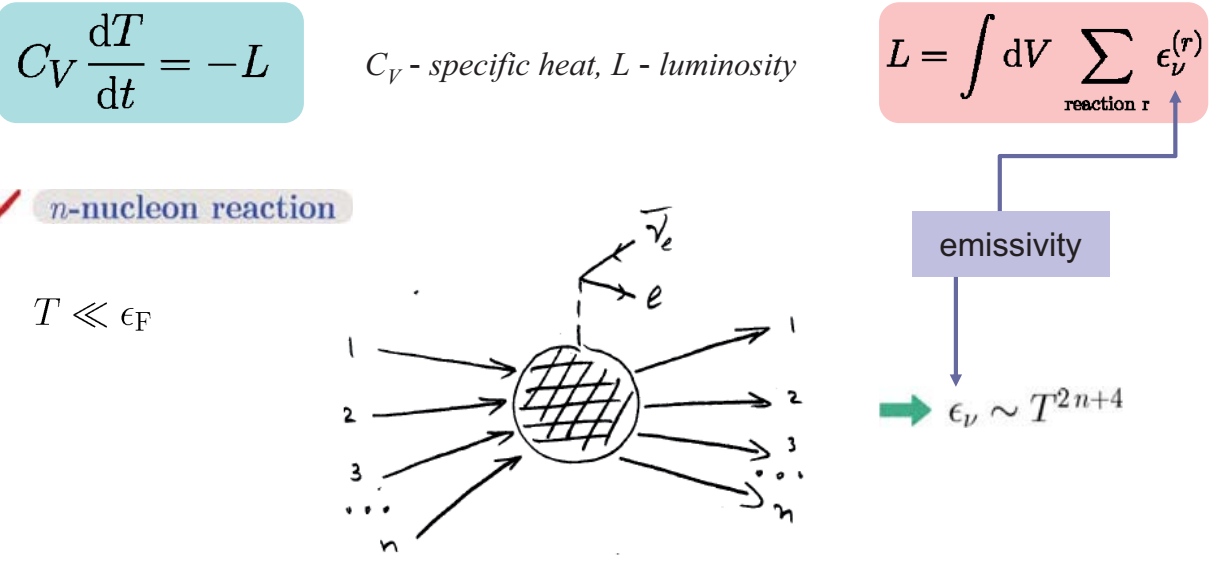

each leg on a Fermi surface $\propto T$

neutrino phase space $\times$ neutrino energy $\quad \omega_{\bar{\nu}} \times \delta\left(\omega_{\bar{\nu}}-\ldots\right) \omega_{\bar{\nu}}^{2} d \omega_{\bar{\nu}} \sim T^{3}$

\section{Direct reactions}

Cooling: role of crust and interior?

most important are reactions in the interior

(The baryon density is $n \gtrsim n_{0} \quad$ where $n_{0}$ is the nuclear saturation density)

$$
\begin{array}{llll}
\text { one-nucleon reactions: } & n \rightarrow p+e+\bar{\nu} & \text { direct URCA (DU) } & \sim \mathrm{T}^{6} \\
\text { two-nucleon reactions: } & n+n \rightarrow n+p+e+\bar{\nu} & \text { modified URCA (MU) } & \sim \mathrm{T}^{8} \\
& n+n \rightarrow n+n+\nu+\bar{\nu} & \text { nucleon bremsstrahlung (NB) }
\end{array}
$$




\section{NS cooling in a nutshell}

black body radiation

$$
L_{\gamma}=4 \pi R^{2} \sigma_{\mathrm{SB}} T_{\text {ext }}^{4}=7.8 \cdot 10^{43} T_{\text {ext }, 9}^{4} \frac{\mathrm{erg}}{\mathrm{s}}
$$

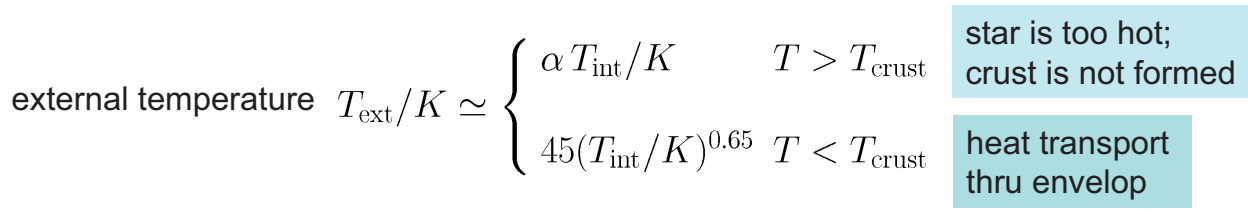

$$
\begin{gathered}
C_{V}=C \cdot T \\
C=10^{30} \frac{\mathrm{erg}}{\mathrm{K}^{2}}=10^{48} \frac{\mathrm{erg}}{\left(10^{9} \mathrm{~K}\right)^{2}} \\
C \int_{T_{0}}^{T(t)} \frac{T d T}{L_{\gamma}(T)}=-t \\
T_{9}=T /\left(10^{9} \mathrm{~K}\right)
\end{gathered}
$$

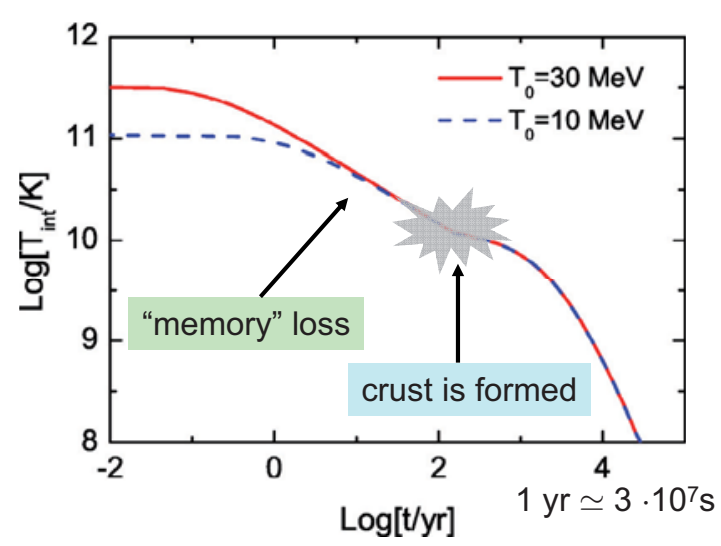

\section{NS cooling in a nutshell}

volume neutrino radiation $\quad T<T_{\text {opac }}=1 \mathrm{MeV}$

$$
\begin{gathered}
\text { DU: } \epsilon_{\mathrm{DU}}=10^{27} T_{9}^{6} \frac{\mathrm{erg}}{\mathrm{s} \mathrm{cm}^{3}} \\
L_{\mathrm{DU}}=V \epsilon_{\mathrm{DU}}=4 \cdot 10^{45} T_{9}^{6} \frac{\mathrm{erg}}{\mathrm{s}} \\
\mathrm{MU}: \epsilon_{\mathrm{MU}}=2 \cdot 10^{21} T_{9}^{8} \frac{\mathrm{erg}}{\mathrm{s} \mathrm{cm^{3 }}} \\
L_{\mathrm{MU}}=V \epsilon_{\mathrm{MU}}=8 \cdot 10^{39} T_{9}^{8} \frac{\mathrm{erg}}{\mathrm{s}}
\end{gathered}
$$

$V=\frac{4 \pi}{3} R^{3}=\frac{4 \pi}{3}(10 \mathrm{~km})^{3}=4 \cdot 10^{18} \mathrm{~cm}^{3}$

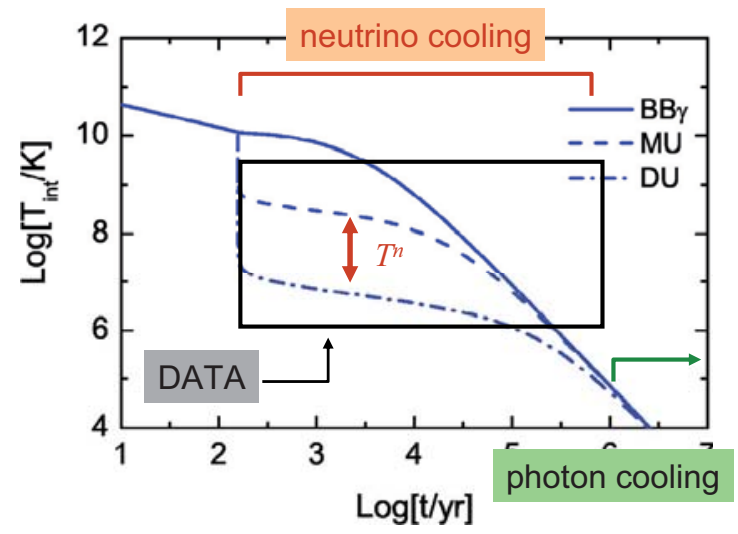


EPJ Web of Conferences

We consider, first, how to calculate the basic neutrino production processes.

No in-medium effects are taken into account

In-medium effects will be studied in lectures 2 and 3

in terms of Green's function formalism

\section{Weak interactions}

Low-energy weak interaction of nucleons

effective weak coupling constant $\quad G=1.16 \cdot 10^{-5} \mathrm{GeV}^{-2}$

nucleon current

lepton current $\quad l_{\mu}=\bar{u}\left(q_{1}\right) \gamma_{\mu}\left(1-\gamma_{5}\right) u\left(q_{2}\right)$

$j_{\mu}=V_{\mu}-A_{\mu}=\#\left(\bar{N} \gamma_{\mu} N\right)-\#\left(\bar{N} \gamma_{\mu} \gamma_{5} N\right)$ 


\section{Weak interactions}

Weinberg's Lagrangian:

$$
\begin{aligned}
& \mathcal{L}=\frac{g}{\sqrt{2}}\left(J_{\mu}^{-} W_{\mu}^{+}+J_{\mu}^{+} W_{\mu}^{-}\right)+\frac{g}{\cos \theta_{W}}\left(J_{\mu}^{(3)}-\sin ^{2} \theta_{W} J_{\mu}^{\text {e.m. }}\right) Z_{\mu}+g \sin \theta_{W} J_{\mu}^{\text {e.m. }} A_{\mu} \\
& \text { lepton current } \quad l_{\mu}=\bar{u}\left(q_{1}\right) \gamma_{\mu}\left(1-\gamma_{5}\right) u\left(q_{2}\right) \\
& \underline{\text { nucleon current }}<N\left|j_{\mu}\right| N>=V_{\mu}^{N N}-A_{\mu}^{N N}=\bar{g}_{V}\left(\bar{N} \gamma_{\mu} N\right)-\bar{g}_{A}\left(\bar{N} \gamma_{\mu} \gamma_{5} N\right) \\
& V_{\mu}^{n p} \approx g_{V} \chi_{p}^{\dagger}\left(p^{\prime}\right)(1, \boldsymbol{v}) \chi_{n}(p) \\
& V_{\mu}^{n n} \approx-\frac{g_{V}}{2} \chi_{n}^{\dagger}\left(p^{\prime}\right)(1, \boldsymbol{v}) \chi_{n}(p) \\
& A_{\mu}^{n p}=-2 A_{\mu}^{p p}=-2 A_{\mu}^{n n} \\
& \approx g_{A} \chi_{p}^{\dagger}\left(p^{\prime}\right)(\boldsymbol{\sigma} \cdot \boldsymbol{v}, \boldsymbol{\sigma}) \chi_{n}(p) \\
& V_{\mu}^{p p} \approx+\frac{g_{V}}{2} \mathrm{c}_{\mathrm{v}} \chi_{p}^{\dagger}\left(p^{\prime}\right)(1, \boldsymbol{v}) \chi_{p}(p) \\
& g_{A}=1.26 \\
& g_{V}=1 \quad \boldsymbol{v}=\frac{\boldsymbol{p}+\boldsymbol{p}^{\prime}}{2 m_{N}} \\
& \mathrm{c}_{\mathrm{v}}=1-4 \sin ^{2} \theta_{W} \simeq 0.08
\end{aligned}
$$

\section{One-nucleon processes (DU)}

emissivity:

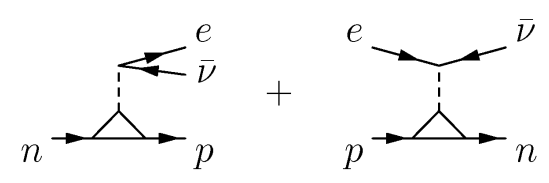

$$
\epsilon_{\nu}^{\mathrm{DU}}=2 \int \frac{d^{3} p_{n}}{(2 \pi)^{3}} f_{n} \int \frac{d^{3} p_{p}}{(2 \pi)^{3}}\left(1-f_{p}\right) \int \frac{d^{3} q_{e}}{2 \omega_{e}(2 \pi)^{3}}\left(1-f_{e}\right) \int \frac{d^{3} q_{\bar{\nu}} \omega_{\bar{\nu}}}{2 \omega_{\bar{\nu}}(2 \pi)^{3}}(2 \pi)^{4} \delta^{(4)}\left(P_{f}-P_{i}\right) \sum_{\text {spins }}|M|^{2}
$$

\section{$\underline{\text { matrix element }}$}

$\sum_{\text {spins }}|M|^{2}=\frac{G^{2}}{2}\left[g_{V}^{2} \operatorname{Tr}\left\{l^{0} l^{0}\right\} \operatorname{Tr}\{\mathbf{1}\}+g_{A}^{2} \operatorname{Tr} \operatorname{Tr}(\boldsymbol{l} \cdot \boldsymbol{\sigma})(\boldsymbol{l} \cdot \boldsymbol{\sigma})\right] \simeq 8 G^{2} \omega_{e} \omega_{\nu}\left(1+3 g_{A}^{2}\right)$

$$
\begin{aligned}
& \operatorname{Tr} l^{\mu} l^{\dagger \nu}=8\left[q_{1}^{\mu} q_{2}^{\nu}+q_{1}^{\nu} q_{2}^{\mu}-\left(q_{1} \cdot q_{2}\right) g^{\mu \nu}-i \epsilon^{\mu \nu \eta \rho}\left(q_{1}\right)_{\eta}\left(q_{2}\right)_{\rho}\right] \\
& \operatorname{Tr} l^{0} l^{\dagger 0}=8\left[\omega_{1} \omega_{2}+\boldsymbol{q}_{1} \boldsymbol{q}_{2}\right] \quad \sum_{i}^{3} \operatorname{Tr} l^{i} l^{\dagger i}=8\left[3 \omega_{1} \omega_{2}-\boldsymbol{q}_{1} \boldsymbol{q}_{2}\right]
\end{aligned}
$$


EPJ Web of Conferences

\section{phase space integration}

$$
\begin{aligned}
& \text { simplifications for } \quad T \ll \epsilon_{F n}, \epsilon_{F p} \quad \omega_{\bar{\nu}}=q_{\bar{\nu}} \sim T \\
& \begin{aligned}
d^{3} p_{i} \simeq d^{3} p_{i} \int d E_{i} \delta\left(E_{i}-E_{p i}\right) \simeq d^{3} p_{i} \int d E_{i} \delta\left(E_{i}-E_{\mathrm{F} i}\right) \\
\delta\left(E_{i}-E_{\mathrm{F} i}\right) \simeq \frac{m_{i}}{p_{\mathrm{F} i}} \delta\left(p_{i}-p_{\mathrm{F} i}\right)
\end{aligned}
\end{aligned}
$$

angle integration

$$
A_{\mathrm{DU}}=\int d \Omega_{n} d \Omega_{p} d \Omega_{e} \delta\left(\boldsymbol{p}_{n}-\boldsymbol{p}_{p}-\boldsymbol{p}_{e}\right)=\frac{8 \pi^{2}}{p_{\mathrm{F} e} p_{\mathrm{F} p} p_{\mathrm{F} n}} \Theta\left(p_{\mathrm{F} p}+p_{\mathrm{F} e}-p_{\mathrm{F} n}\right)
$$

triangle inequality

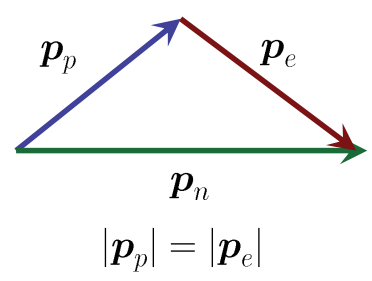

critical condition

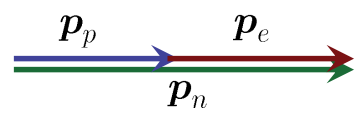

\section{energy integration}

since the integration over energy goes from $-\infty$ to $+\infty$ and $\quad 1-f(-E)=f(E)$ under integral we can replace $\quad 1-f_{i} \Longrightarrow f_{i}$

$$
I=\int_{0}^{+\infty} d x_{\bar{\nu}} x_{\bar{\nu}}^{3} \prod_{j=1}^{3} \int_{-\infty}^{+\infty} d x_{j} f_{j}\left(x_{j}\right) \delta\left(x_{1}-x_{2}-x_{3}-x_{\bar{\nu}}\right)=\frac{457 \pi^{6}}{5040}
$$

$$
\epsilon_{\nu}^{\mathrm{DU}}=\frac{457 \pi}{10080} G^{2}\left(1+3 g_{A}^{2}\right) m_{n} m_{p} p_{\mathrm{F}, e} T^{6} \Theta\left(2 p_{\mathrm{F} p}-p_{\mathrm{F} n}\right)
$$




\section{One-nucleon processes (DU)}

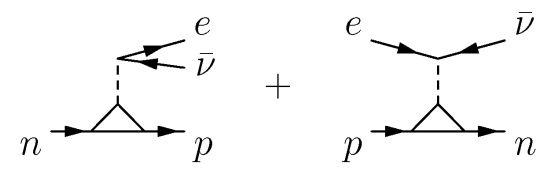

allowed if $\left|p_{\mathrm{F}, n}-p_{\mathrm{F}, p}\right|<p_{\mathrm{F}, e} \longrightarrow$ proton concentration $>11-14 \%$

$$
\epsilon_{\nu}^{\mathrm{DU}} \simeq 4 \cdot 10^{27}\left(\frac{n_{e}}{n_{0}}\right)^{1 / 3} T_{9}^{6} \Theta\left(2 p_{\mathrm{F}, p}-p_{\mathrm{F}, n}\right) \frac{\mathrm{erg}}{\mathrm{cm}^{3} \cdot \mathrm{s}}
$$

- large prefactor $\sim 10^{27}-10^{28}$

- weak temperature dependence $T^{6}$

BUT does not always occur

$$
n>n_{c}^{\mathrm{DU}}\left(M>M_{c}^{\mathrm{DU}}\right)
$$

\section{One-nucleon processes on neutral currents}

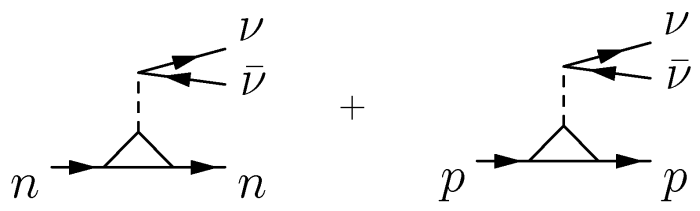

energy-momentum conservation

$$
\begin{aligned}
& \delta\left(\boldsymbol{p}_{1}-\boldsymbol{p}_{2}-\boldsymbol{q}_{1}-\boldsymbol{q}_{2}\right) \delta\left(E_{1}-E_{2}-\omega_{1}-\omega_{2}\right) \\
& \begin{aligned}
E_{1}=E\left(p_{1}\right)=\frac{p_{1}^{2}}{2 m_{N}} & =\frac{\left(\boldsymbol{p}_{1}-\boldsymbol{q}_{1}-\boldsymbol{q}_{2}\right)^{2}}{2 m_{N}}+\omega_{1}+\omega_{2} \\
& \approx \frac{p_{1}^{2}}{2 m_{N}}-v_{F}\left|\boldsymbol{q}_{1}+\boldsymbol{q}_{2}\right| \cos \theta+\left|\boldsymbol{q}_{1}\right|+\left|\boldsymbol{q}_{2}\right|
\end{aligned}
\end{aligned}
$$

requires $v_{\mathrm{F}} \geq 1$ 


\section{Process on $\pi^{-}$condensate (PU)}

$$
\text { assume } \mu_{e} \text { reaches } m_{\pi} \begin{gathered}
e^{-} \rightarrow \pi^{-} \\
n \rightarrow p+\pi^{-} \quad \text { Bose condensate of pions }
\end{gathered}
$$

neutrons in both initial and final states

$$
\begin{gathered}
A_{\mathrm{PU}}=\int d \Omega_{n_{1}} d \Omega_{n_{2}} d \Omega_{\pi} \delta\left(\boldsymbol{p}_{n_{1}}-\boldsymbol{p}_{n_{2}}-\boldsymbol{p}_{e}\right) \simeq \frac{8 \pi^{2}}{p_{\mathrm{F}, n}^{2} p_{\mathrm{F}, e}} \cdot 1 \\
\boldsymbol{p}_{n}
\end{gathered} \boldsymbol{p}_{e}
$$

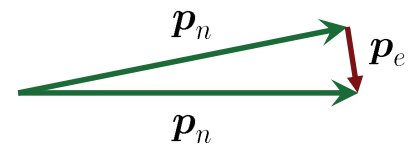

$\Rightarrow$ energy-momentum conservation is easily fulfilled

[Bahcall Wolf, Phys. Rev. (1965) ]

\section{Process on pion condensate (PU)}

Migdal's pion condensate $\mathrm{k}=\left(\mu_{\pi}, \mathrm{k}_{\mathrm{c}}\right): \mu_{\pi}<\mathrm{m}_{\pi}, \mathrm{k}_{\mathrm{c}} \sim \mathrm{p}_{\mathrm{F}, \mathrm{e}} \quad p$-wave condensate several processes are possible
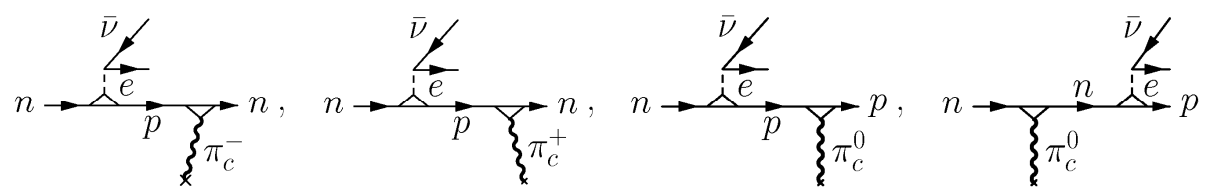

$$
\epsilon_{\nu} \sim 10^{26} T_{9}^{6}\left(n / n_{0}\right)^{1 / 3} \frac{\mathrm{erg}}{\mathrm{cm}^{3} \mathrm{sec}}
$$

Kaon condensate processes yield a smaller contribution $\left[\propto \sin ^{2} \theta_{\mathrm{C}} \simeq(0.23)^{2}\right]$ All "exotic" processes start only when the density exceeds some critical density

In order to calculate these reactions we have to introduce new diagrammatic elements: 


\section{Intermediate fermion propagator}

\section{outgoing proton:}

$$
\begin{array}{r}
G=\frac{1}{E+\omega+\mu_{n}-E_{n}\left(p_{1}\right)} \approx \frac{1}{\omega+\mu_{n}-\mu_{p}} \\
\quad \text { since } \quad E \approx E_{p}(p)-\mu_{p}, \quad \text { for } \quad v_{\mathrm{F}}^{N} \ll 1
\end{array}
$$

\section{incoming neutron}

$$
\begin{aligned}
& G=\frac{1}{E-\omega+\mu_{p}-E_{p}(\boldsymbol{p}-\boldsymbol{q})}=-\frac{1}{\omega+\mu_{n}-\mu_{p}} \\
& \text { ince } \quad E=E_{n}(p)-\mu_{n}, \quad \text { for } \quad v_{\mathrm{F}}^{N} \ll 1
\end{aligned}
$$

\section{neutral processes}
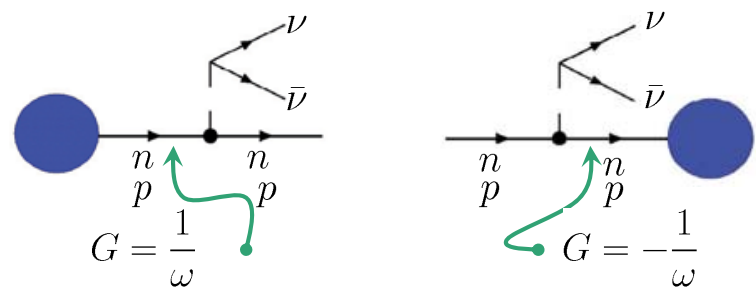

\section{$\pi N N$ Vertices}

$$
H_{\mathrm{int}}=f_{\pi N N} \psi_{N}^{\dagger} \sigma_{i} \tau_{a} \psi_{N}\left(\nabla_{i} \varphi_{a}\right)
$$

$\left(\varphi_{1}, \varphi_{2}, \varphi_{3}\right) \quad$ three neutral pseudoscalar fields $\psi_{N}=\left(\begin{array}{c}\psi_{p} \\ \psi_{n}\end{array}\right)$ nucleon isospin dublet

$\sigma_{1}, \sigma_{2}, \sigma_{3}$ Pauli matrices acting in spin space

$\tau_{1}, \tau_{2}, \tau_{3} \quad$ Pauli matrices acting in isospin space

$$
\begin{gathered}
\tau_{a} \varphi_{a}=\tau_{1} \varphi_{1}+\tau_{2} \varphi_{2}+\tau_{3} \varphi_{3}=\sqrt{2} \tau_{+} \pi^{+}+\sqrt{2} \tau_{-} \pi^{-}+\tau_{3} \pi^{0} \\
\pi^{ \pm}=\frac{\varphi_{1} \mp i \varphi_{2}}{\sqrt{2}}, \quad \pi^{0}=\varphi_{3} \text { pion annihilation operators } \\
\tau_{ \pm}=\frac{\tau_{1} \pm i \tau_{2}}{2} \text { rising and lowering isospin matrices }
\end{gathered}
$$

$$
H_{\text {int }}=f_{\pi N N}\left\{\sqrt{2} \psi_{p}^{\dagger} \sigma \psi_{n} \nabla \pi^{+}+\sqrt{2} \psi_{n}^{\dagger} \sigma \psi_{p} \nabla \pi^{-}+\psi_{p}^{\dagger} \sigma \psi_{p} \nabla \pi^{0}-\psi_{n}^{\dagger} \sigma \psi_{n} \nabla \pi^{0}\right\}
$$


EPJ Web of Conferences

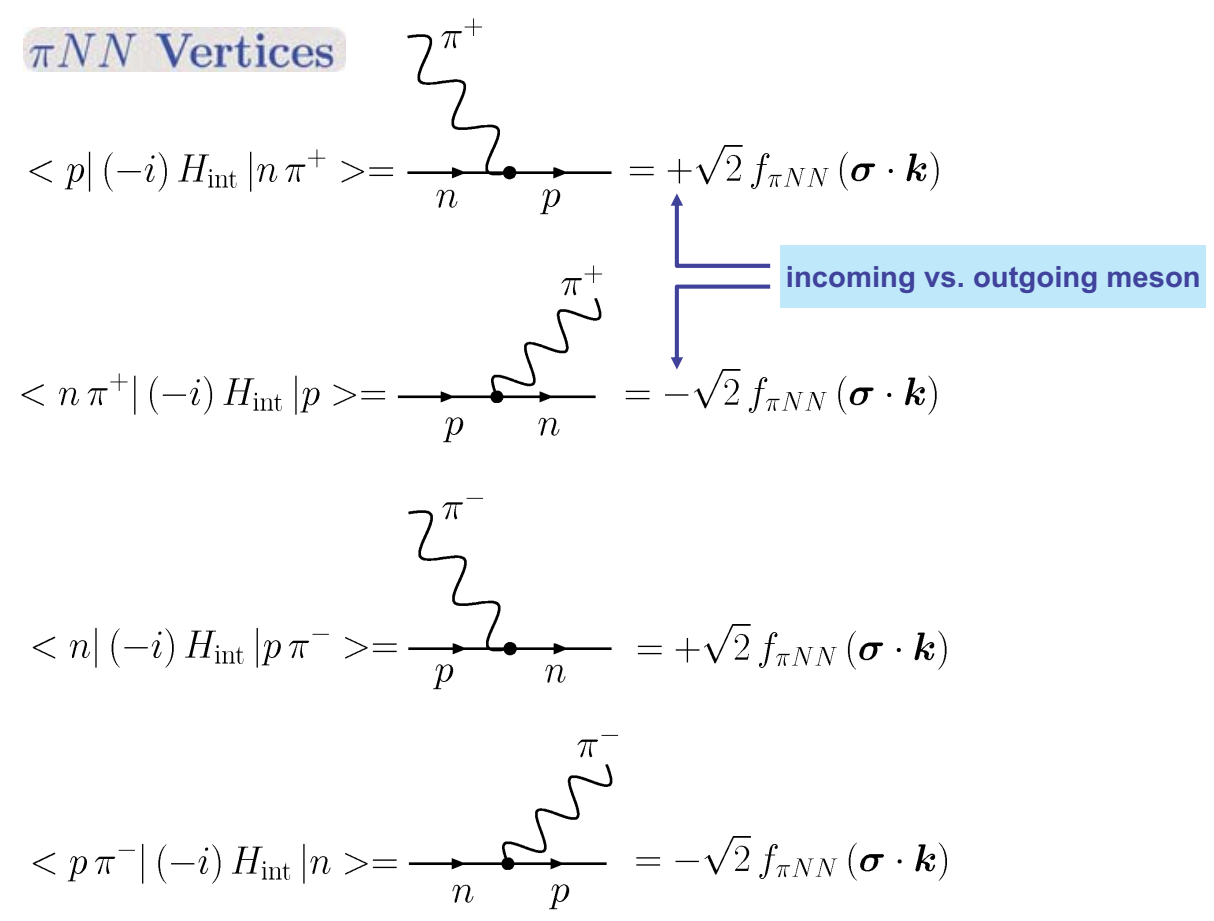

\section{$\pi N N$ Vertices}
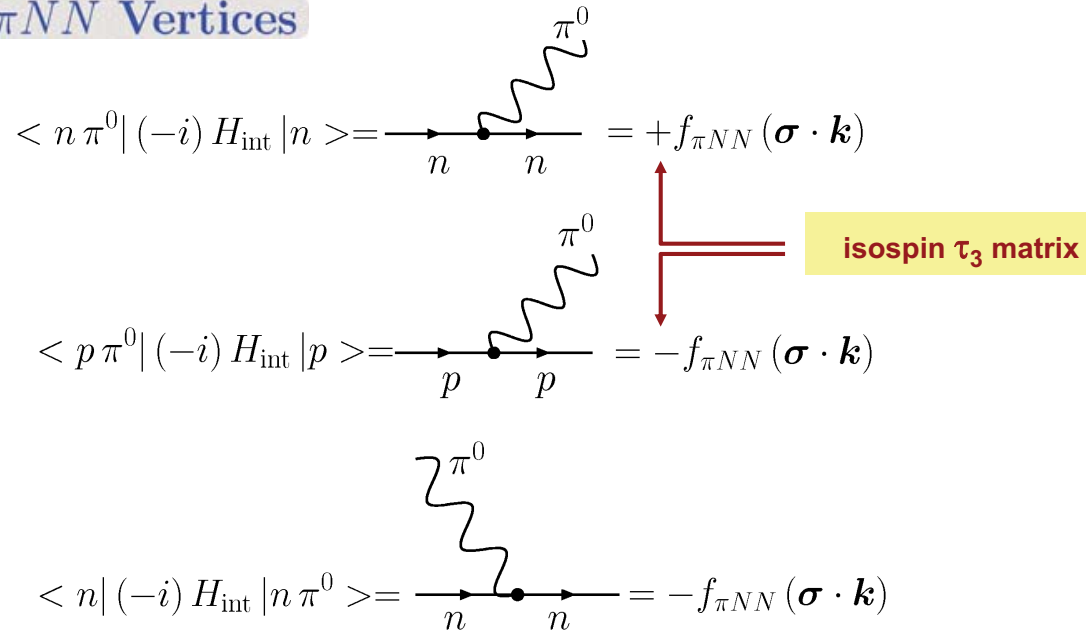

$<p\left|(-i) H_{\text {int }}\right| p \pi^{0}>=\stackrel{2_{p}^{\pi^{0}} \cdot \vec{p}}{\vec{p}}=+f_{\pi N N}(\boldsymbol{\sigma} \cdot \boldsymbol{k})$ 


\section{Emissivity of PU reaction on $\pi^{-}$condensate}

Maxwell et al., AJ (1977)

\section{PU process operates for $\boldsymbol{n}>\boldsymbol{n}_{\boldsymbol{c}}{ }^{\pi}$}

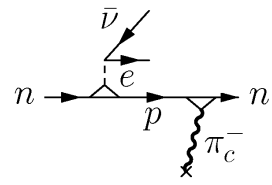

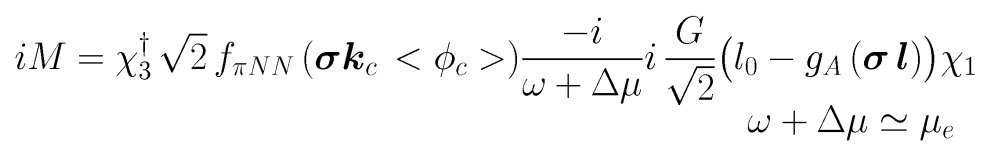

Condensate field $\quad \phi_{c}=a e^{-i \omega_{c} t+i \boldsymbol{k}_{c} \boldsymbol{r}} \quad$ with fixed momentum and frequency and amplitude $a$

$$
\text { Using: } \quad \operatorname{Tr}\left\{\sigma_{i} \sigma_{j}\right\}=2 \delta_{i j} \quad \sum_{\text {spins }}|M|^{2}=\frac{16 f_{\pi N N}^{2}\left(1+3 g_{A}^{2}\right) G^{2} k_{c}^{2}}{(\omega+\Delta \mu)^{2}} \omega_{1} \omega_{2} a^{2}
$$

Inversed reaction gives extra factor 2

$$
\begin{aligned}
& \epsilon_{\nu}^{\pi_{c}^{-}}= \frac{457 \pi}{5040} G^{2}\left(1+3 g_{A}^{2}\right) m_{n}^{2} p_{\mathrm{F}, e} a_{\pi}^{2} T^{6} \Theta\left(n-n_{c}\right) \quad a_{\pi}^{2}=\frac{<|\pi|^{2}>}{4 f_{\pi}^{2}} \\
& \quad \text { condensate amplitude } \\
& a^{2} \sim(0.01-0.1) \\
& \quad \Rightarrow \quad \epsilon_{\nu}^{-} \sim(0.1 \div 1) \epsilon_{\nu}^{\mathrm{DU}}
\end{aligned}
$$

\section{Emissivity of PU reaction on $\pi^{0}$ condensate}

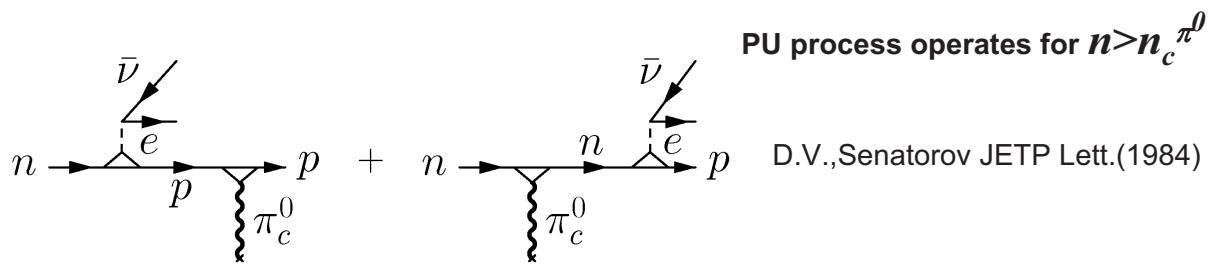

$$
\begin{aligned}
i M= & \chi_{3}^{\dagger} f_{\pi N N}\left(\boldsymbol{\sigma} \boldsymbol{k}_{c}\right)<\phi>\frac{i}{-\omega} \frac{i G}{\sqrt{2}}\left(l_{0}-g_{A}(\boldsymbol{\sigma} \boldsymbol{l})\right) \chi_{1} \\
& -\chi_{3}^{\dagger} \frac{i G}{\sqrt{2}}\left(l_{0}-g_{A}(\boldsymbol{\sigma} \boldsymbol{l})\right) \frac{i}{\omega} f_{\pi N N}\left(\boldsymbol{\sigma} \boldsymbol{k}_{c}\right)<\phi>\chi_{1} \\
= & +2 \frac{f_{\pi N N} G}{\sqrt{2} \omega} \chi_{3}^{\dagger}\left(\boldsymbol{\sigma} \boldsymbol{k}_{c}<\phi>l_{0}-g_{A} \boldsymbol{l} \boldsymbol{k}_{c}<\phi>\right) \chi_{1}
\end{aligned}
$$

$\phi=a \cos \left(\boldsymbol{k}_{c} \boldsymbol{r}\right) \quad \sum_{\text {spins }}|M|^{2}=32 f_{\pi N N}^{2}\left(1+g_{A}^{2}\right) G^{2} k_{c}^{2} \frac{\omega_{1} \omega_{2}}{\omega^{2}} a^{2} \underbrace{\left\langle\sin ^{2}\left(\boldsymbol{k}_{c} \boldsymbol{r}\right)>\right.}_{=\frac{1}{2}}$ Inversed reaction gives extra factor 2

$$
\epsilon_{\nu}^{\pi_{c}^{0}}=\frac{457 \pi}{5040} G^{2}\left(1+g_{A}^{2}\right) f_{\pi N N}^{2} m_{n} m_{p} k_{c} a^{2} T^{6} \sim \epsilon_{\nu}^{\pi_{c}^{-}}
$$




\section{Two-nucleon process (MU)}

$$
n+p \rightarrow p+p+e+\bar{\nu} \quad n+n \rightarrow n+p+e+\bar{\nu}
$$

$\checkmark$ no critical density

$\checkmark 5$ fermions $\Rightarrow$ suppressed phase-space volume (compared to one-nucleon processes)

$\checkmark T^{8}$ dependence of the emissivity

$\left(5\right.$ fermions $\left.\quad \Rightarrow \sim T^{5}, \quad \omega_{\bar{\nu}} \delta\left(\omega_{\bar{\nu}}+\ldots\right) \omega_{\bar{\nu}}^{2} d \omega_{\bar{\nu}} \quad \Rightarrow T^{3}\right)$

\section{Two-nucleon process (Modified Urca)}

Friman \& Maxwell AJ (1979) $\quad n+n \rightarrow n+p+e+\bar{\nu}$

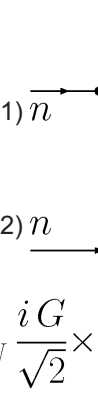

$$
\begin{aligned}
& \vec{n} \sum \vec{n} \stackrel{<}{<} \stackrel{e}{\cdot p} \\
& \sum_{i} \pi^{0} n \\
& i M=f_{\pi N N}^{2} \frac{i G}{\sqrt{2}} \times \\
& \begin{array}{l}
e \\
\bar{\nu}
\end{array} \\
& \left\{\chi_{3}^{\dagger}\left[(-\boldsymbol{\sigma} \boldsymbol{k})\left(l_{0}-g_{A} \boldsymbol{l} \boldsymbol{\sigma}\right)\right] \chi_{1} \frac{i^{2} D_{\pi^{0}}\left(\omega_{\pi}, k\right)}{-(\omega+\Delta \mu)} \chi_{4}^{\dagger}(-\boldsymbol{\sigma} \boldsymbol{k}) \chi_{2}\right. \\
& +\chi_{3}^{\dagger}\left[\left(l_{0}-g_{A} \boldsymbol{l} \boldsymbol{\sigma}\right)(+\boldsymbol{\sigma} \boldsymbol{k})\right] \chi_{1} \frac{i^{2} D_{\pi^{0}}\left(\omega_{\pi}, k\right)}{+(\omega+\Delta \mu)} \chi_{4}^{\dagger}(-\boldsymbol{\sigma} \boldsymbol{k}) \chi_{2} \\
& \left.+\chi_{3}^{\dagger} \sqrt{2}(-\boldsymbol{\sigma} \boldsymbol{k}) \chi_{1} \frac{i^{2} D_{\pi^{-}}\left(\omega_{\pi}, k\right)}{-(\omega+\Delta \mu)} \chi_{4}^{\dagger}\left[\sqrt{2}(+\boldsymbol{\sigma} \boldsymbol{k})\left(l_{0}-g_{A} \boldsymbol{l} \boldsymbol{\sigma}\right)\right] \chi_{2}\right\}
\end{aligned}
$$

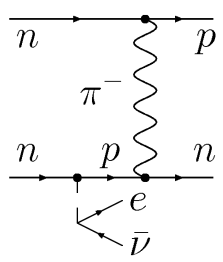




\section{Two-nucleon process (Modified Urca)}

\section{Emissivity:}

$$
\begin{aligned}
\epsilon_{\nu}^{\mathrm{MU}} & =\prod_{i=1}^{4} \int\left[\frac{d^{3} p_{i}}{(2 \pi)^{3}}\right] f_{1} f_{2}\left(1-f_{3}\right)\left(1-f_{4}\right) \frac{d^{3} q_{e}\left(1-f_{e}\right)}{2 \omega_{e}(2 \pi)^{3}} \\
& \times \frac{d^{3} q_{\bar{\nu}}}{2 \omega_{\bar{\nu}}(2 \pi)^{3}} \omega_{\bar{\nu}}(2 \pi)^{4} \delta^{(4)}\left(P_{f}-P_{i}\right) \frac{1}{s} \sum_{\text {spins }}|M|^{2},
\end{aligned}
$$

$s=2$ is symmetry factor. Reactions with the electron in an initial state yield extra factor 2.

Finally

$$
\begin{gathered}
\epsilon_{\nu}^{\mathrm{MU}}=\frac{11513}{60480 \pi} G^{2} g_{A}^{2} f_{\pi N N}^{4} m_{n}^{3} m_{p} p_{\mathrm{F}, e} T^{8} 1.3 \simeq 8 \cdot 10^{21}\left(n_{p} / n_{0}\right)^{1 / 3} T_{9}^{8} \times \frac{\mathrm{erg}}{\mathrm{cm}^{3} \cdot \mathrm{s}} \\
\text { due to exchange reactions }
\end{gathered}
$$

Coherence: only axial-vector term contributes (!)

whereas for PU processes both vector and axial-vector terms contribute

\section{Standard scenario. DU scenario}

standard scenario (MU)

only part of the data can be described

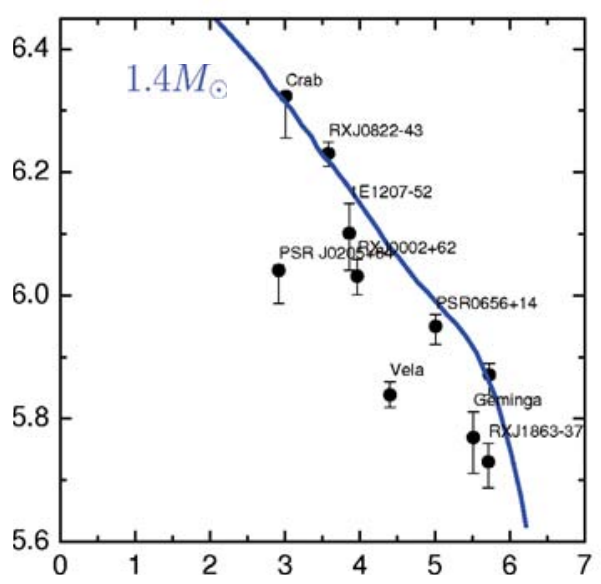

[Blaschke, Grigorian, Voskresensky A\&A (2004) ] 


\section{Standard scenario. DU scenario}

standard scenario (MU)

only part of the data can be described

Direct-Urca scenario

NS masses close to $M_{\text {crit }}^{\text {DU }}$

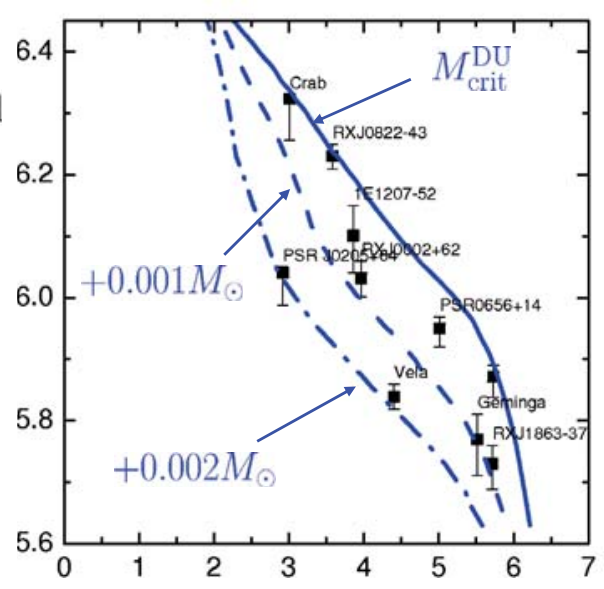

[Blaschke, Grigorian, Voskresensky A\&A (2004) ]

\section{Standard scenario. DU scenario}

standard scenario (MU)

only part of the data can be described

Direct-Urca scenario

NS masses close to $M_{\text {crit }}^{\text {DU }}$

Neutron stars with $M>M_{\text {crit }}^{\text {DU }}$ will be too cold

But masses of NS are not close to each others

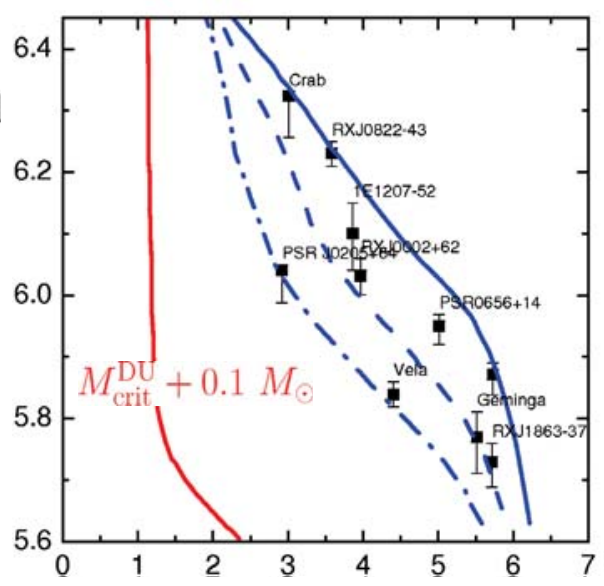

[Blaschke, Grigorian, Voskresensky A\&A (2004)] 


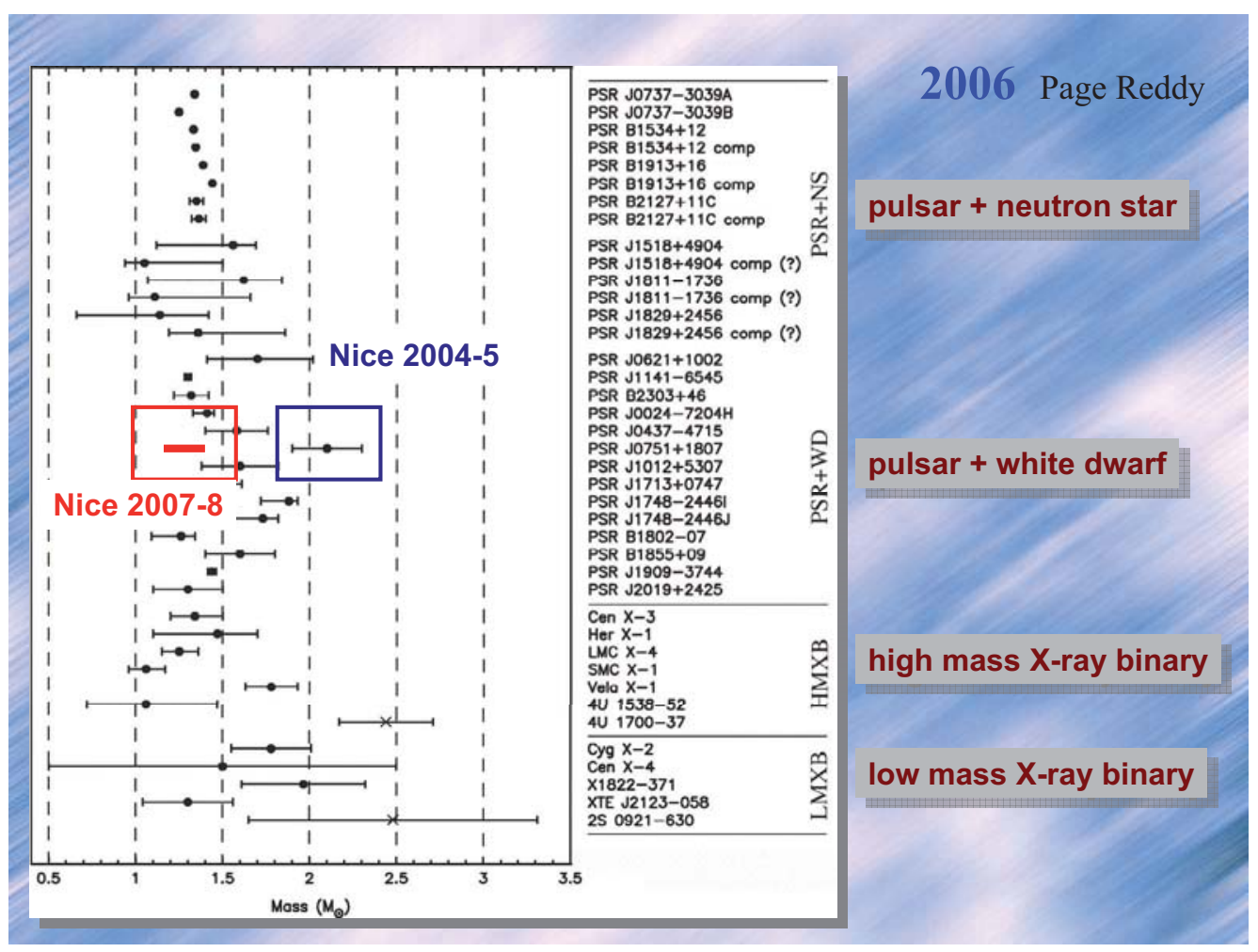

\section{Direct-Urca Cooling Scenario ?}

DU process schould be ,exotics“

(if DU starts it is dificult to stop it)

- $M_{\text {crit }}^{\mathrm{DU}} \gtrsim 1.3 M_{\odot}$

- $\quad n_{\text {crit }}^{\mathrm{DU}} \gtrsim 4 n_{0}$

EoS should produce a large DU threshold in NS matter ! 


\section{DU thresholds}

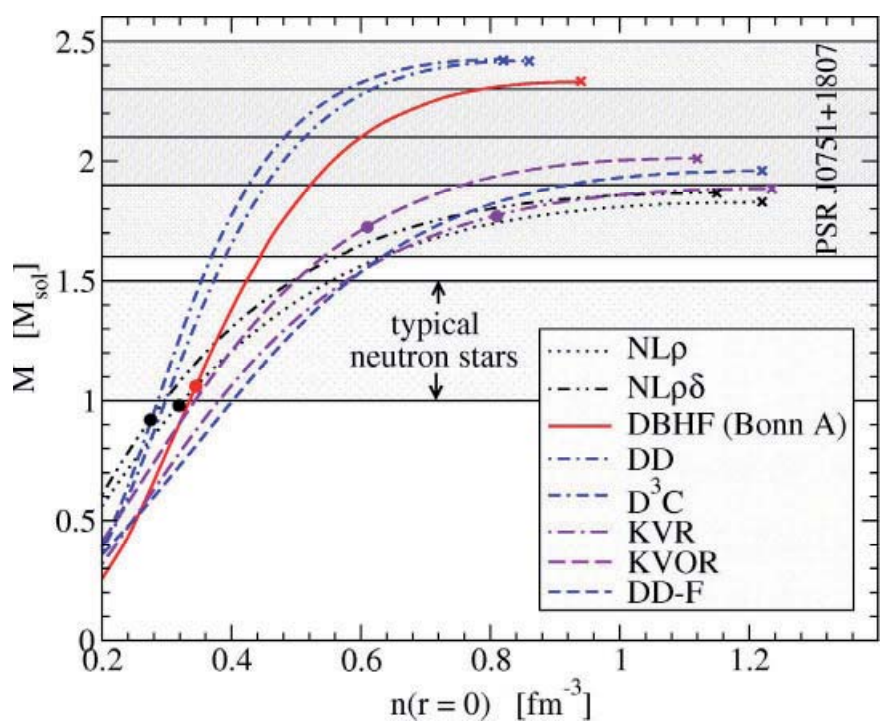

Klähn et al. PRC (2006)

\section{Pairing in nuclear matter}

PHYSICAL REVIEW

VOLUME 110 , NUMBER

M AY 15,1958

Possible Analogy between the Excitation Spectra of Nuclei and Those of the Superconducting Metallic State

A. Bohr, B. R. Mottelson, AND D. Ptnes*

Institute for Theoretical Physics, University of Copenhagen, Copenhagen, Demmark, and Nordisk Institut for Teoretish Atomfysik, Copenhagen, Denmark

$$
\text { (Received January 7, 1958) }
$$

The evidence for an energy gap in the intrinsic excitation spectrum of nuclei is reviewed. A possible analogy between this effect and the energy gap observed in the electronic excitation of a superconducting metal is suggested.

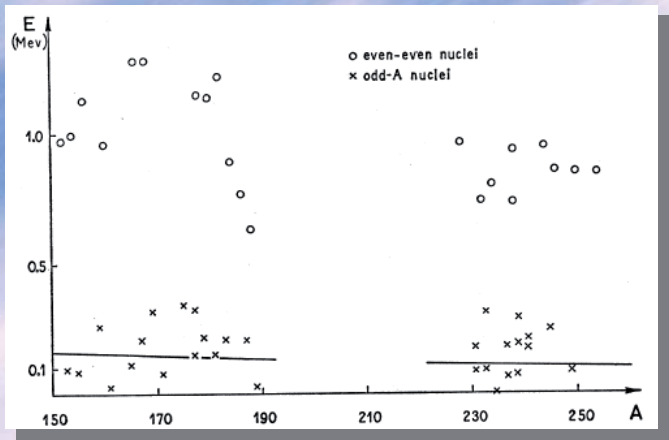




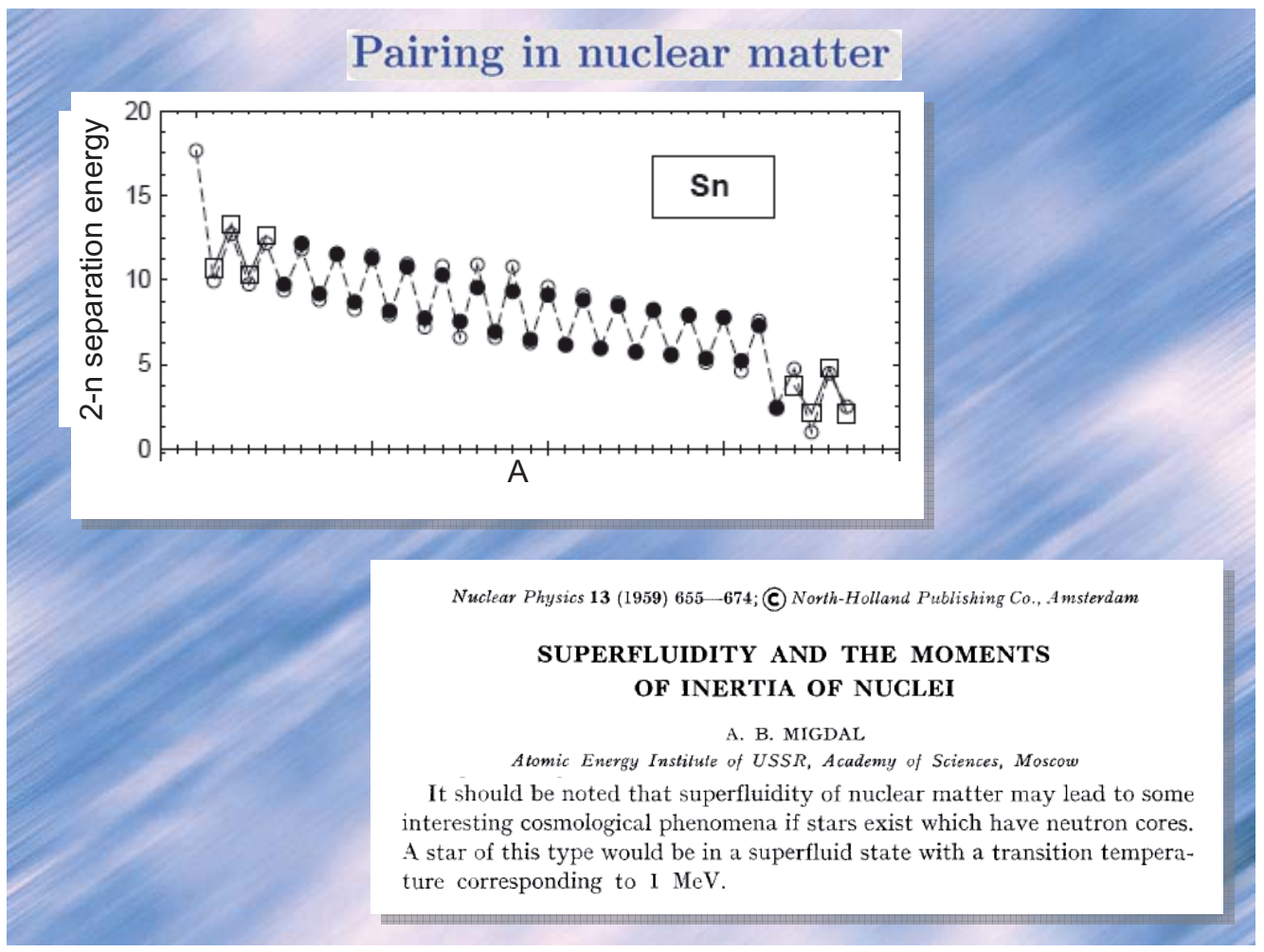

\section{Pairing in nuclear matter}

excitation spectrum

- without pairing

$$
E=\epsilon_{p}-\epsilon_{F} \simeq v_{\mathrm{F}}\left(p-p_{\mathrm{F}}\right)
$$

- with pairing

$$
E= \pm \sqrt{v_{F}^{2}\left(p-p_{\mathrm{F}}\right)^{2}+\Delta^{2}}
$$
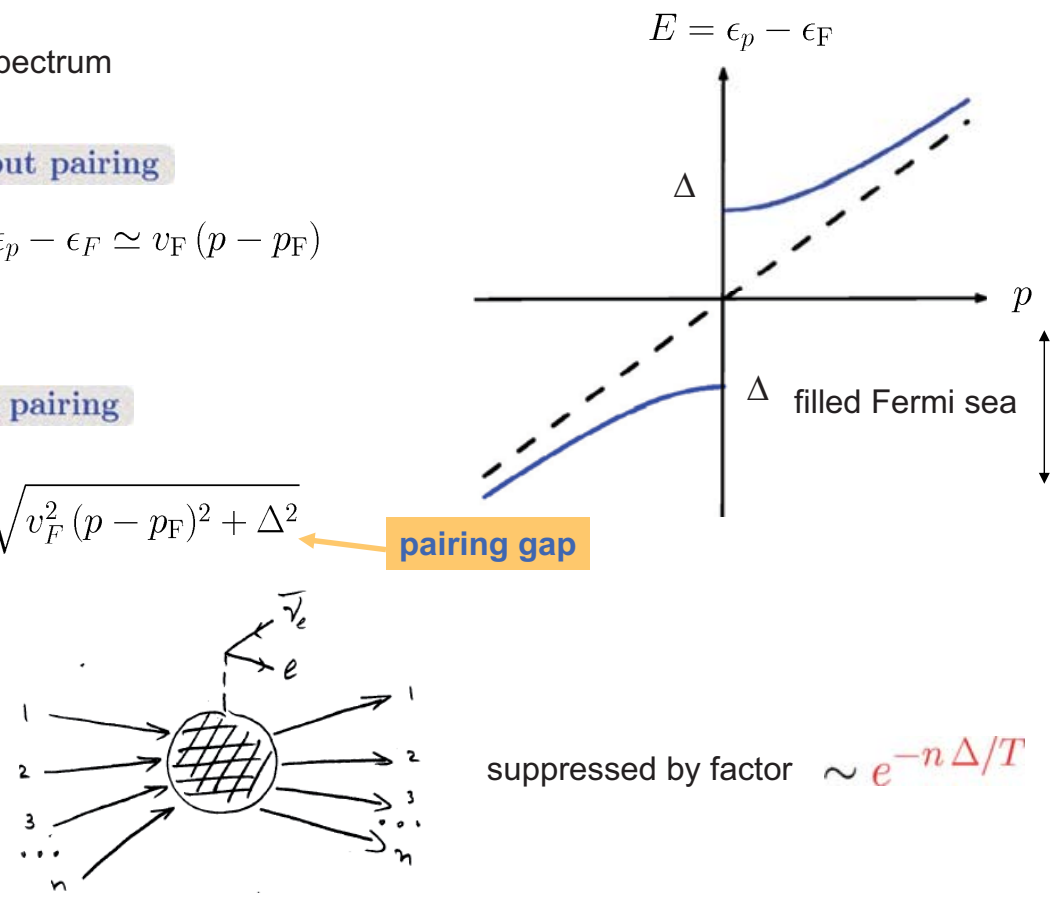

suppressed by factor $\sim e^{-n \Delta / T}$ 


\section{Pairing gaps in nuclear matter}

\section{pairing gaps}

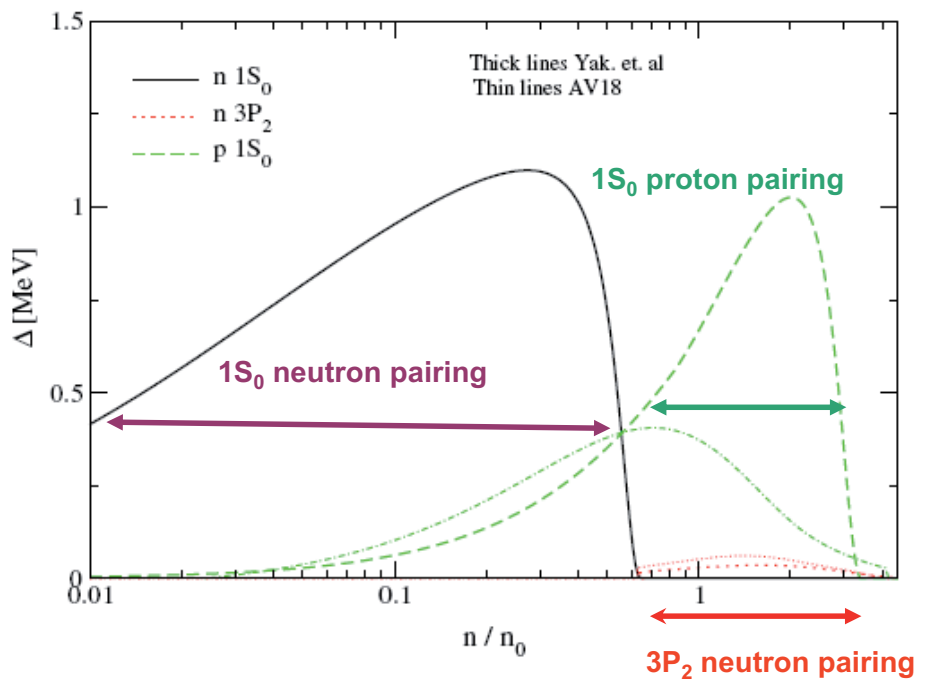

[Schwenk Friman, PRL (2004)]

\section{Breaking and Formation of Cooper pairs (PBF)}

In superfluid $\left(T<T_{c}<0.1-1 \mathrm{MeV}\right)$ all two-nucleon processes are suppressed by factor $\exp (-2 \Delta T)$ new "quasi"-one-nucleon-like processes (one-nucleon phase space volume) become permitted

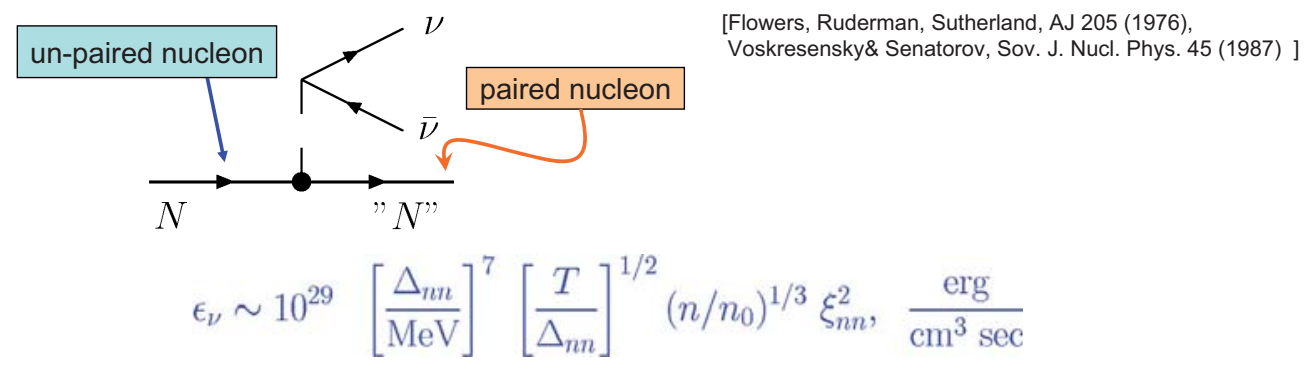

$\Delta_{\mathrm{nn}}$ is neutron gap and $\xi_{\mathrm{nn}}=\exp \left(-\Delta_{\mathrm{nn}} / \mathrm{T}\right)$

[ Voskresensky, Senatorov, Sov. J. Nucl. Phys. 45 (1987); Senatorov, Voskresensky, Phys. Lett. B184 (1987); Voskresensky astro-ph/0101514 ]

not $\epsilon_{\nu} \sim 10^{20} T_{9}^{7} \xi_{n n}^{2} \quad$ as in Flowers et al. (1976)

Naively one expect the emissivity of $p \rightarrow p v v$ to be suppressed by extra $c_{V}^{2} \sim 0.006$ factor. 


\section{Effects of pairing on the neutron star cooling}
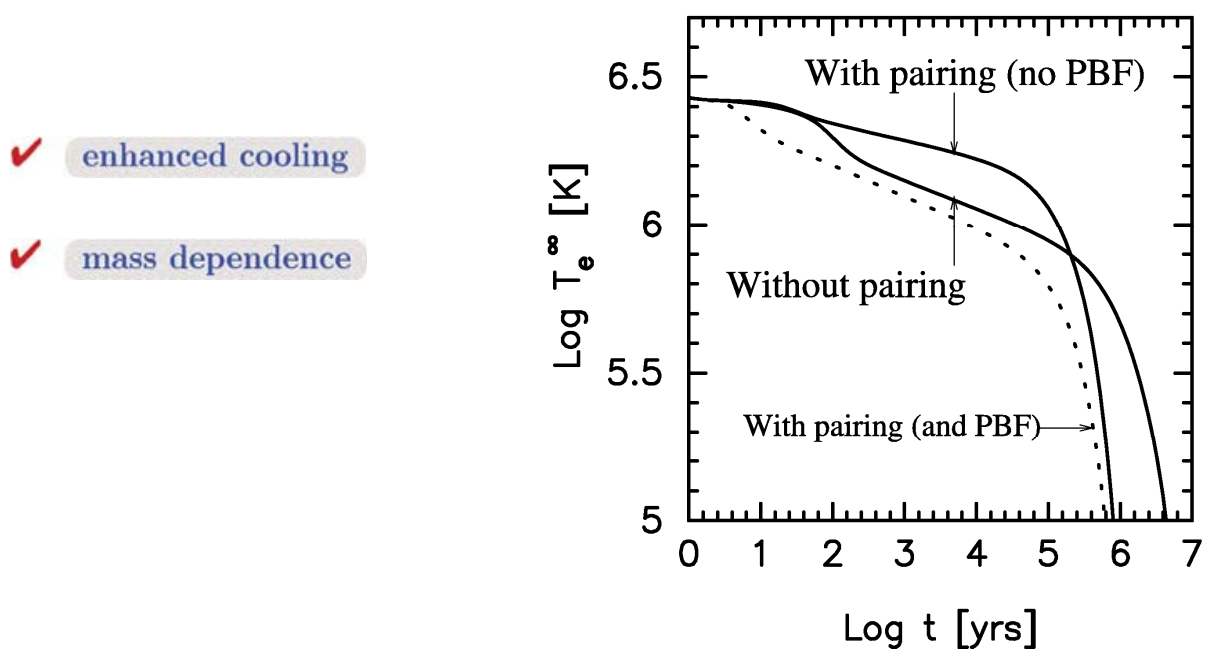

[Page, Geppert, Weber, NPA (2006)]

\section{Neutrino emission reactions}

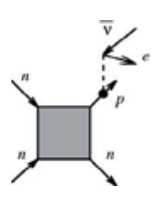

$\underline{\text { standard }} \quad \underline{T<T_{\text {opac }} \sim 10^{-1} \div 10^{0} \mathrm{MeV}}$

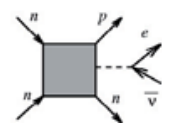

$$
10^{22} \times\left(\frac{m_{N}^{*}}{m_{N}}\right)^{4} T_{9}^{8}\left(\frac{n_{e}}{n_{0}}\right)^{\frac{1}{3}} \frac{\mathrm{erg}}{\mathrm{cm}^{3} \mathrm{~s}} \times e^{-2 \Delta / T}
$$
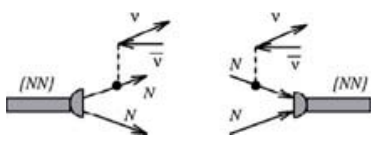

$$
10^{29} \times\left(\frac{m_{N}^{*}}{m_{N}}\right)\left(\frac{\Delta}{\mathrm{MeV}}\right)^{7}\left(\frac{T}{\Delta}\right)^{\frac{1}{2}} e^{-2 \Delta / T} \frac{\mathrm{erg}}{\mathrm{cm}^{3} \mathrm{~s}}
$$

$\underline{\text { exotic }}$

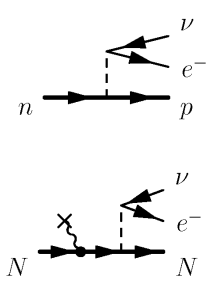

$$
\begin{array}{r}
10^{27} \times\left(\frac{m_{N}^{*}}{m_{N}}\right)^{2} T_{9}^{6}\left(\frac{n_{e}}{n_{0}}\right)^{\frac{1}{3}} \frac{\mathrm{erg}}{\mathrm{cm}^{3} \mathrm{~s}} \times e^{-\Delta / T} \\
\text { allowed if }\left|p_{\mathrm{F}, n}-p_{\mathrm{F}, p}\right|<p_{\mathrm{F}, e} \\
7 \cdot 10^{26} \times\left(\frac{m_{N}^{*}}{m_{N}}\right)^{2} T_{9}^{6} \frac{\left|\varphi_{c}\right|^{2}}{m_{\pi}^{2}} \frac{\mathrm{erg}}{\mathrm{cm}^{3} \mathrm{~s}} \times e^{-\Delta / T}
\end{array}
$$




\section{Green's functions}

$\mathrm{N}$-body system: wave function of the whole system $\Psi\left(\mathrm{x}_{1}, \mathrm{x}_{2}, \ldots, \mathrm{x}_{\mathrm{N}}\right)$

encodes the dynamics of all particles and is very complicated

Introduce the object which describes the dynamics of the reduced number of particles of interest

\section{one-particle propagation}

$$
x^{\prime}=\left(t^{\prime}, \boldsymbol{x}^{\prime}\right)
$$

$$
x=(t, \boldsymbol{x})
$$

Amplitude of particle transition from a point $(x, t)$ to a point $\left(x^{\prime}, t^{\prime}\right)$

$$
\Psi\left(\boldsymbol{x}^{\prime}, t^{\prime}\right)=\int d \boldsymbol{x} G^{(+)}\left(\boldsymbol{x}^{\prime}, t^{\prime} ; \boldsymbol{x}, t\right) \Psi(\boldsymbol{x}, t) \quad t^{\prime}>t
$$

for $t^{\prime}=t+0 \quad \Psi\left(\boldsymbol{x}^{\prime}, t+0\right)=\int d \boldsymbol{x} G^{(+)}\left(\boldsymbol{x}^{\prime}, t+0 ; \boldsymbol{x}, t\right) \Psi(\boldsymbol{x}, t)$

$$
G^{(+)}\left(\boldsymbol{x}^{\prime}, t+0 ; \boldsymbol{x}, t\right)=\delta\left(\boldsymbol{x}^{\prime}-\boldsymbol{x}\right)
$$

If $\Psi(\boldsymbol{x}, t)$ obeys the Schrödinger equation $\quad\left[i \partial_{t}-H(\boldsymbol{x})\right] \Psi(\boldsymbol{x}, t)=0$

$$
\left[i \partial_{l}-H(\boldsymbol{x})\right] G^{(+)}\left(\boldsymbol{x}, t ; \boldsymbol{x}^{\prime}, t^{\prime}\right)=i \delta\left(t-t^{\prime}\right) \delta\left(\boldsymbol{x}-\boldsymbol{x}^{\prime}\right)
$$

for homogeneous system : $\quad G^{(+)}\left(\boldsymbol{x}^{\prime}, t^{\prime} ; \boldsymbol{x}, t\right)=G^{(+)}\left(\left(\boldsymbol{x}^{\prime}-\boldsymbol{x}\right)^{2}, t^{\prime}-t>0\right)$

eigenfunctions: $\quad H \varphi_{\lambda}(\boldsymbol{x})=\epsilon_{\lambda}(\boldsymbol{x}) \varphi(\boldsymbol{x})$

$$
G^{(+)}\left(\boldsymbol{x}^{\prime}, \boldsymbol{x}, \tau=t^{\prime}-t\right)=-\sum_{\lambda} \int_{-\infty}^{\infty} \frac{d \epsilon}{2 \pi i} e^{-i \epsilon \tau} \frac{\varphi_{\lambda}\left(\boldsymbol{x}^{\prime}\right) \varphi_{\lambda}^{*}(\boldsymbol{x})}{\epsilon-\epsilon_{\lambda}+i 0}
$$

$$
\begin{gathered}
G^{(+)}\left(\boldsymbol{x}^{\prime}, t^{\prime} ; \boldsymbol{x}, t\right)=<N\left|\hat{\Psi}\left(\boldsymbol{x}^{\prime}, t^{\prime}\right) \hat{\Psi}^{\dagger}(\boldsymbol{x}, t)\right| N> \\
\hat{\Psi}(\boldsymbol{x}, t)=\sum_{\lambda} \varphi_{\lambda}(x) \hat{a}_{\lambda} e^{-i \epsilon_{\lambda} t} \quad\left|N>=a_{a}^{\dagger} a_{2}^{\dagger} a_{3}^{\dagger} \ldots a_{N}^{\dagger}\right| 0>
\end{gathered}
$$




\section{Green's function of non-interacting fermions}

$$
\begin{aligned}
i G\left(\boldsymbol{x}, t ; \boldsymbol{x}^{\prime}, t^{\prime}\right) & =<N\left|T\left\{\hat{\Psi}(\boldsymbol{x}, t) \hat{\Psi}^{\dagger}\left(\boldsymbol{x}^{\prime}, t^{\prime}\right)\right\}\right| N> \\
& =<N\left|\hat{\Psi}(\boldsymbol{x}, t) \hat{\Psi}^{\dagger}\left(\boldsymbol{x}^{\prime}, t^{\prime}\right)\right| N>-<N\left|\hat{\Psi}^{\dagger}\left(\boldsymbol{x}^{\prime}, t^{\prime}\right) \hat{\Psi}(\boldsymbol{x}, t)\right| N>
\end{aligned}
$$

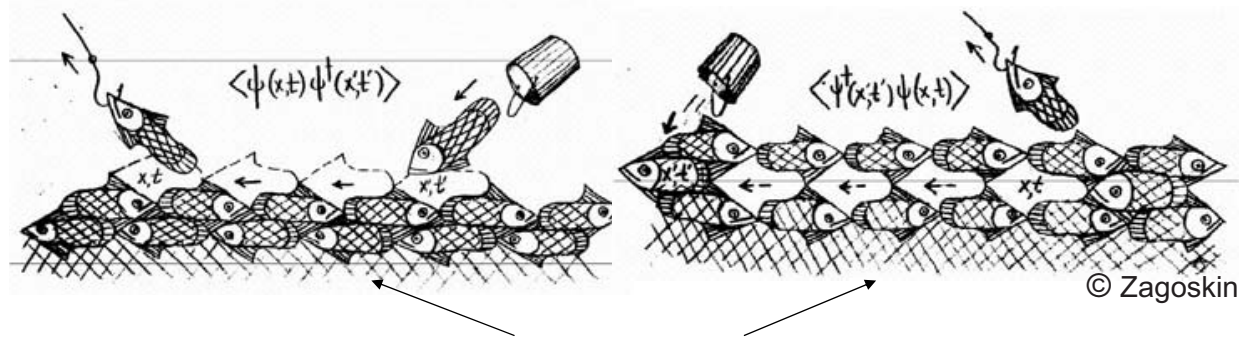

$$
G(\epsilon, p)=\frac{1-\mathrm{n}_{\mathrm{p}}}{\epsilon-\epsilon_{p}+i 0}+\frac{\mathrm{n}_{\mathrm{p}}}{\epsilon+\epsilon_{p}^{h}-i 0}
$$

$T=0$

$$
\begin{aligned}
& n_{p}=\theta\left(p_{\mathrm{F}}-p\right) \\
& \epsilon_{p}^{h}=-\epsilon_{p}
\end{aligned}
$$$$
G(\epsilon, \boldsymbol{p})=\frac{1}{\epsilon-\epsilon_{p}+i \operatorname{sign}\left(\epsilon-\epsilon_{\mathrm{F}}\right)}
$$

\section{Diagram technique}

Ground state:

$$
i G(x, y)=<N\left|\widehat{T}\left\{\widehat{\Psi}(x) \widehat{\Psi}^{\dagger}(y)\right\}\right| N>=<N\left|\widehat{S}^{-1} \widehat{T}\left\{\widehat{\Psi}_{I}(x) \widehat{\Psi}_{I}^{\dagger}(y)\right\} \widehat{S}\right| N>
$$

in interaction picture: $\quad i G=<N\left|\widehat{T}\left\{\widehat{\Psi}_{I}(x) \widehat{\Psi}_{I}^{\dagger}(y)\right\} \widehat{S}\right| N><\widehat{S}^{-1}>$

transition from the ground state to the ground state under action of evolution operator

$$
\begin{gathered}
\widehat{S}=\underset{\uparrow}{\widehat{T}} \exp \left\{-i \int_{-\infty}^{\infty} \widehat{V}_{I}(t) d t\right\} \\
\text { time ordering }
\end{gathered}
$$

$$
\begin{array}{r}
\widehat{V}_{I}(t)=e^{i \widehat{H}_{0}(\mu) t} \widehat{V} e^{-i \widehat{H}_{0}(\mu) t} \\
\widehat{H}_{0}(\mu)=H_{0}-\sum_{a} \mu_{a} \widehat{N}_{a}
\end{array}
$$

Only one type of Green's functions 
particle

$$
\mathrm{G}(\varepsilon, \mathrm{p})
$$

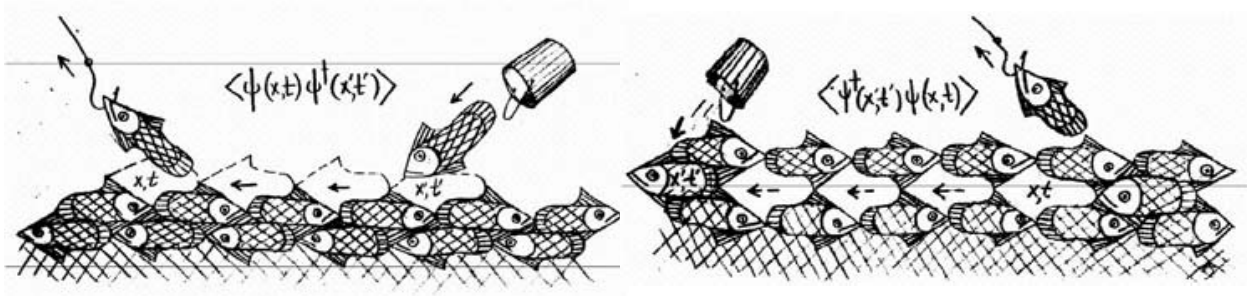

hole

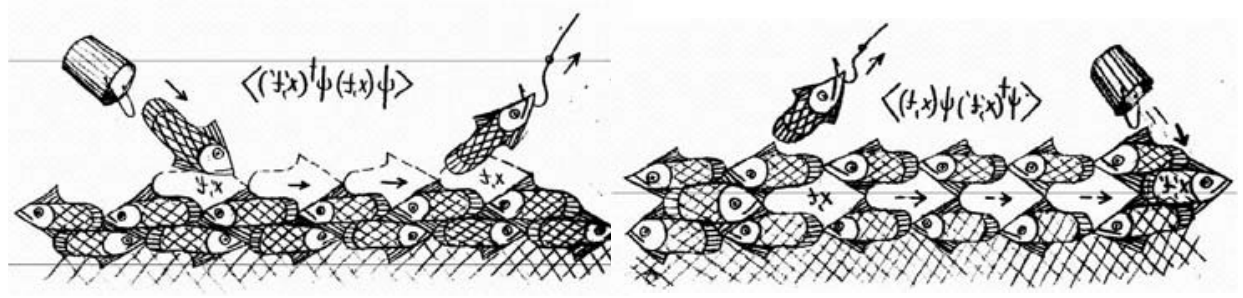

$\mathrm{G}^{\mathrm{h}}(\varepsilon, \mathrm{p})$

\section{Diagram technique "out of non-equilibrium"}

For a non-equilibrium state $\mid N>$

$$
\begin{aligned}
i G^{--}(x, y)=<N\left|\widehat{T}\left\{\widehat{\Psi}(x) \widehat{\Psi}^{\dagger}(y)\right\}\right| N> & =<N\left|\widehat{S}^{-1} \widehat{T}\left\{\widehat{\Psi}_{I}(x) \widehat{\Psi}_{I}^{\dagger}(y)\right\} \widehat{S}\right| n> \\
& \neq<N\left|\widehat{T}\left\{\widehat{\Psi}_{I}(x) \widehat{\Psi}_{I}^{\dagger}(y)\right\} \widehat{S}\right| N><\widehat{S}^{-1}>
\end{aligned}
$$

non-equilibrium ground state at $-\infty$ does not transit to the same ground state at $+\infty$

due to possible decays $\mathbf{4}$ Green's functions

$$
i G^{--}(x, y)=<N\left|\widehat{T}\left\{\widehat{\Psi}(x) \widehat{\Psi}^{\dagger}(y)\right\}\right| N>i G^{++}(x, y)=<N\left|\widehat{T^{\dagger}}\left\{\widehat{\Psi}(x) \widehat{\Psi}^{\dagger}(y)\right\}\right| N>
$$

Wigner densities (no time ordering operations)

$$
i G^{-+}(x, y)=\mp<N\left|\widehat{\Psi}^{\dagger}(y) \widehat{\Psi}(x)\right| N>\quad i G^{+-}(x, y)=<N\left|\widehat{\Psi}(x) \widehat{\Psi}^{\dagger}(y)\right| N>
$$

Green functions are not independent !

$$
G^{--}+G^{++}=G^{-+}+G^{+-} \text {. }
$$




\section{Diagram technique "out of non-equilibrium"}

Assume suppression of initial correlations $\left(t>>t_{c o r}\right) \Rightarrow$ Wick theorem

$\Rightarrow$ averaging of equations of motion for operators we obtain coupled Schwinger-Dyson equations

$$
\begin{aligned}
& \text { for } G^{--}, G^{++}, G^{+-}, G^{-+} \\
& G(x, y)=G^{0}(x, y)+\int G^{0}(x, z) \Pi\left(z, z^{\prime}\right) G\left(z^{\prime}, y\right) \\
& \text { matrix of matrix of matrix of } \\
& \text { full G.F. bare G.F. self-energies }
\end{aligned}
$$

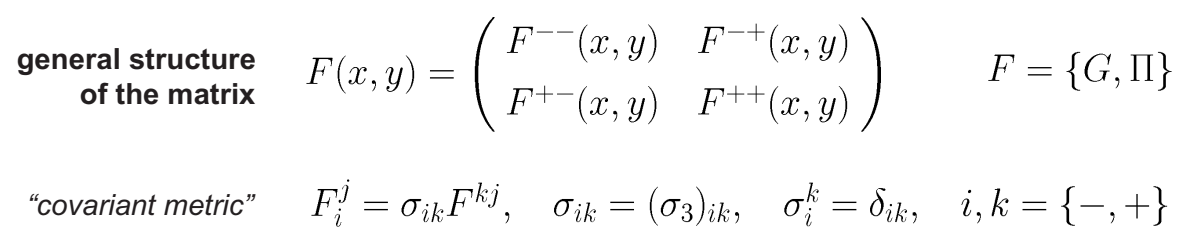

[Ivanov, Knoll, Voskresensky. NPA 657 (1999); NPA 672 (2000)]

different notations compared with LP (different signs in $G^{i j}$ and $\Pi^{i j}$ ) and KB text books $(>,<$ )

Factor $(-i)$ for " - "vertex and $(+i)$ for " + " vertex is used.

- retarded Green's function $G^{R}=G^{--}-G^{-+}$

decouples and defines excitation spectrum

$$
G_{12}^{R}=G_{12}^{0, R}+G_{13}^{0, R} \cdot \Pi_{34}^{R} \cdot G_{42}^{R}
$$

- No Wick rotation

- Same diagrams as for ground-state system 


\section{Thermal equilibrium}

In equilibrium only one Green's function $\left(G^{R}\right)$ is required :

$F(p)=\left(\begin{array}{cc}F^{R} \pm i f(E) \mathcal{A} & \pm i f(E) \mathcal{A} \\ -i(1 \mp f(E)) \mathcal{A} & -F^{A} \mp i f(E) \mathcal{A}\end{array}\right)$ for Green's functions $\quad \begin{aligned} \mathcal{A} & =A \\ \text { for self-energies } & \mathcal{A}=\Gamma\end{aligned}$

particle occupation factors: $f(E)=\frac{1}{e^{(E / T)} \pm 1}$ Wigner densities: $\quad G^{-+} \propto f(E)$ spectral function: $A(p)=-2 \operatorname{Im} G^{R}=\frac{\Gamma}{M^{2}+\Gamma^{2} / 4} \quad$ width $\quad \Gamma=-2 \operatorname{Im} \Pi^{R}$ mass operator $\quad M=-Q(p)+\operatorname{Re} \Pi \quad$ with $\quad Q=\epsilon-\frac{\boldsymbol{p}^{2}}{2 m} \quad \begin{aligned} & \text { non-relativistic } \\ & \text { particles }\end{aligned}$ $Q=p^{2}-m^{2} \quad \begin{array}{ll}\text { relativistic } \\ \text { bosons }\end{array}$

\section{Thermal equilibrium}

fermion Green's function $\quad\left(\begin{array}{cc}G^{R}+i f(E) A & +i f(E) A \\ -i(1-f(E)) A & -G^{A}-+i f(E) A\end{array}\right)$

$\Rightarrow G^{-+} \propto f(E) ! \quad \Rightarrow$ specific role of $G^{-+}$

- Quasiparticle limit $\quad \Gamma \rightarrow 0 \quad A \rightarrow 2 \pi \delta(M)$

that fixes in-medium mass-shell $\quad M=-Q(p)+\operatorname{Re} \Pi=0$

in quasiparticle limit $T \ll E_{\mathrm{F}}$ each extra $G^{-+}$line brings a factor $\propto \frac{T^{2}}{E_{\mathrm{F}}^{2}}$

"perturbation series" in number of (- +) Green functions (T-counting)

- gas limit particles are on mass-shell and $\operatorname{Re} \Pi^{R} \rightarrow 0$

$G_{0}^{-+}=+2 \pi i f(E) \delta\left(E+\mu-E_{p}\right) \quad G_{0}^{+-}=-2 \pi i(1-f(E)) \delta\left(E+\mu-E_{p}\right)$ 
momentum distribution of quantum kinetic equation

$$
f(\boldsymbol{p})= \pm \int i G^{-+}(E, \boldsymbol{p}) \frac{d E}{2 \pi}
$$

$\Rightarrow G^{-+}(E, \boldsymbol{p})$ has the meaning of a generalized distributions of virtual particles In general case - no relation between $E$ and $\boldsymbol{p}$ !

\section{Optical theorem in quantum mechanics}

In quantum mechanics $\quad \sigma_{\text {tot }}=\frac{4 \pi}{k} \operatorname{Im} F(0) \longleftarrow$ forward scattering amplitude.

$$
\operatorname{Im} F\left(\boldsymbol{k}^{\prime}, \boldsymbol{k}\right)=\frac{k}{4 \pi} \int F\left(\boldsymbol{k}^{\prime}, \boldsymbol{k}^{\prime}\right) F^{*}\left(\boldsymbol{k}^{\prime \prime}, \boldsymbol{k}\right) d \Omega^{\prime \prime}
$$

Scattering amplitude in the Born approximation

$$
f^{\mathrm{B}}(q)=-\frac{m}{2 \pi} \int e^{-i \boldsymbol{q} \boldsymbol{r}} U(\boldsymbol{r}) d^{3} \boldsymbol{r}, \quad q=2 k \sin \frac{\theta}{2}
$$

$\Rightarrow \operatorname{Im} f^{\mathrm{B}}(\theta=0)=0$ and the optical theorem is not fulfilled.

Solution: $f^{\mathrm{B}}$ is the first term in the perturbative expansion of $f$ in $U$. Since $\sigma \propto|f|^{2} \Rightarrow \sigma \propto U^{2}$ for small $U$.

Therefore in optical theorem $\left[\sigma \propto \operatorname{lm} f^{\mathrm{B}}(\theta=0)\right]$ there is no linear term.

$$
\Rightarrow \operatorname{Im} f^{\mathrm{B}}(\theta=0) \propto U^{2} \Rightarrow \text { higher order term is required (!) }
$$




\section{Optical theorem in non-equilibrium diagram technique}

$$
\begin{aligned}
& \text { 作, } \\
& -i \Pi_{0}^{-+}=\frac{G^{2}}{2} \operatorname{Tr}\left\{l_{1}^{\mu} l_{2}^{\nu}\right\} \int \frac{d^{4} p}{(2 \pi)^{4}} \operatorname{Tr}\left\{\left(-i J_{\mu}\right) \underline{i G_{n}^{-+}(p+q)}\left(+i J_{\nu}\right) i \underline{G_{p}^{+-}(p)(-1)}\right\} \\
& =-i \mathcal{L}_{0}^{-+} \sum_{\text {spin }}|M|^{2} \quad 2 \pi i f(E+\omega) \delta\left(E+\omega+\mu-E_{p+q}^{(n)}\right)-2 \pi i(1-f(E)) \delta\left(E+\mu-E_{\mathrm{p}}\right)
\end{aligned}
$$
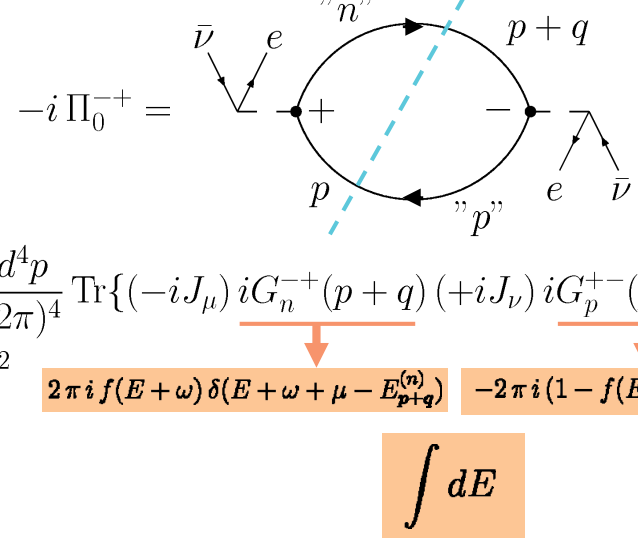

Cutting the diagram means removing of $d E$ integration due to $\delta$-function

Comparing with standard expression for emissivity

$$
\begin{gathered}
\epsilon_{\nu}^{\mathrm{DU}}=2 \int \frac{d^{3} p_{n}}{(2 \pi)^{3}} f_{n} \frac{d^{3} p_{p}}{(2 \pi)^{3}}\left(1-f_{p}\right) \frac{d^{3} q_{e}}{2 \omega_{e}(2 \pi)^{3}}\left(1-f_{e}\right) \frac{d^{3} q_{\bar{\nu}} \omega_{\bar{\nu}}}{2 \omega_{\bar{\nu}}(2 \pi)^{3}}(2 \pi)^{4} \delta^{(4)}\left(P_{f}-P_{i}\right) \sum_{\text {spins }}|M|^{2} \\
\epsilon_{\nu}^{\mathrm{DU}}=2 \int \frac{d^{3} q_{e}}{2 \omega_{e}(2 \pi)^{3}}\left(1-f_{e}\right) \frac{d^{3} q_{\bar{\nu}}}{2 \omega_{\bar{\nu}}(2 \pi)^{3}} \omega_{\bar{\nu}}\left[-i \Pi_{0}^{-+}\left(q_{e}+q_{\bar{\nu}}\right)\right]
\end{gathered}
$$

to calculate Direct Urca emissivity

$$
\text { we need only (no medium effects) simple free W boson "- +" loop }
$$

Using relation $\quad i \Pi^{-+}=-\frac{2 \operatorname{Im} \Pi^{R}}{e^{\omega / T}-1} \begin{aligned} & \text { we may calculate cross-sections as an integral of } \\ & |M|^{2} \text { over the phase space } \mathbf{O R} \text { as an imaginary part }\end{aligned}$ of $\mathrm{W}^{\wedge}$ - boson self-energy

perturbative expansion: second-order term in weak coupling and zeroth-order term in strong coupling

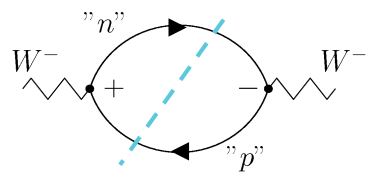

Terms of higher order in strong couplings must be included! $\Pi_{0}^{-+} \longrightarrow \Pi^{-+}$ 
- Bose occupation number out of fermion loop

$$
\begin{gathered}
f_{\mathrm{F}}\left(E_{1}\right)\left[1-f_{\mathrm{F}}\left(E_{2}\right)\right]=\left[f_{\mathrm{F}}\left(E_{2}\right)-f_{\mathrm{F}}\left(E_{1}\right)\right] f_{\mathrm{B}}\left(E_{1}-E_{2}\right) \\
-i \mathcal{L}_{0}^{-+}=\int \frac{d^{3} p}{(2 \pi)^{3}} f_{\mathrm{F} n}(\boldsymbol{p}+\boldsymbol{q})\left[1-f_{\mathrm{F} p}(\boldsymbol{p})\right] 2 \pi \delta\left[E^{n}(\boldsymbol{p}+\boldsymbol{q})-\omega-E^{p}(p)-\mu_{n}+\mu_{p}\right] \\
=f_{B}(\omega) \int \frac{d^{3} p}{(2 \pi)^{3}}\left[f_{\mathrm{F} p}(\boldsymbol{p})-f_{\mathrm{F} n}(\boldsymbol{p}+\boldsymbol{q})\right] 2 \pi \delta\left[E^{n}(\boldsymbol{p}+\boldsymbol{q})-\omega-E^{p}(p)-\mu_{n}+\mu_{p}\right] \\
=-2 f_{B}(\omega) \operatorname{Im} \int \frac{d^{3} p}{(2 \pi)^{3}} \frac{f_{\mathrm{F} p}(\boldsymbol{p})-f_{\mathrm{F} n}(\boldsymbol{p}+\boldsymbol{q})}{E^{n}(\boldsymbol{p}+\boldsymbol{q})-\omega-E^{p}(p)-\mu_{n}+\mu_{p}-i 0} \\
=-f_{B}(\omega) 2 \operatorname{Im} \mathcal{L}_{n p}^{R} \\
\mathcal{L}_{a b}^{R}=\int \frac{d^{3} p}{(2 \pi)^{3}} \frac{f_{\mathrm{F} a}(\boldsymbol{p}+\boldsymbol{q})-f_{\mathrm{F} b}(\boldsymbol{p})}{\omega+E^{b}(p)-E^{a}(\boldsymbol{p}+\boldsymbol{q})+\mu_{a}-\mu_{b}+i 0}
\end{gathered}
$$

Lindhard function

very sharp function of $\omega$ and $\kappa$

$$
\begin{aligned}
\mathcal{L}_{a b}^{R} & =\int \frac{d^{3} p}{(2 \pi)^{3}} \frac{f_{\mathrm{F} a}(\boldsymbol{p}+\boldsymbol{q})-f_{\mathrm{F} b}(\boldsymbol{p})}{\omega+E^{b}(p)-E^{a}(\boldsymbol{p}+\boldsymbol{q})+\mu_{a}-\mu_{b}+i 0} \\
& =-\frac{m_{N}}{2 \pi^{2}}\left[p_{\mathrm{F}, a} \Phi_{1, a}(\omega, k)+p_{\mathrm{F}, b} \Phi_{1, b}(-\omega,-k)\right]
\end{aligned}
$$

Migdal function $(T=0)$ :

$$
\begin{aligned}
& \text { function }(T=0) \text { : } \\
& \begin{array}{ll}
\Phi_{1, a}(\omega, k)=\frac{m_{N}^{2}}{2 p_{\mathrm{F}, a} k^{3}}\left\{\frac{a^{2}-b^{2}}{2} \ln \frac{a+b}{a-b}-a b\right\} & \begin{array}{l}
a=\omega-\frac{k^{2}}{2 m_{N}} \\
b=\frac{p_{\mathrm{F}, a} k}{m_{N}}
\end{array} \\
|a| \ll b \quad \Phi_{1, a} \simeq \frac{1}{2} & \\
|a| \gg b \quad \Phi_{1, a} \simeq-\frac{\pi^{2} n_{a}}{m_{N}^{2} p_{\mathrm{F}, a}} \frac{1}{\omega-\frac{k^{2}}{2 m_{N}}}
\end{array}
\end{aligned}
$$




\section{Optical theorem for modified URCA reactions}

$$
\epsilon_{\nu}^{\mathrm{MU}}=\int \frac{d^{3} q_{e}\left(1-f_{e}\right)}{2 \omega_{e}(2 \pi)^{3}} \frac{d^{3} q_{\bar{\nu}}}{2 \omega_{\bar{\nu}}(2 \pi)^{3}} \omega_{\bar{\nu}}\left[-i \Pi_{\mathrm{MU}}^{-+}\left(q_{e}+q_{\bar{\nu}}\right)\right]
$$

To get correct 2-order $\Pi^{-+}$one should add diagrams with $\pi^{-}$ corresponding to $n p \rightarrow p p e \bar{\nu}$ reaction. They should be added coherently.
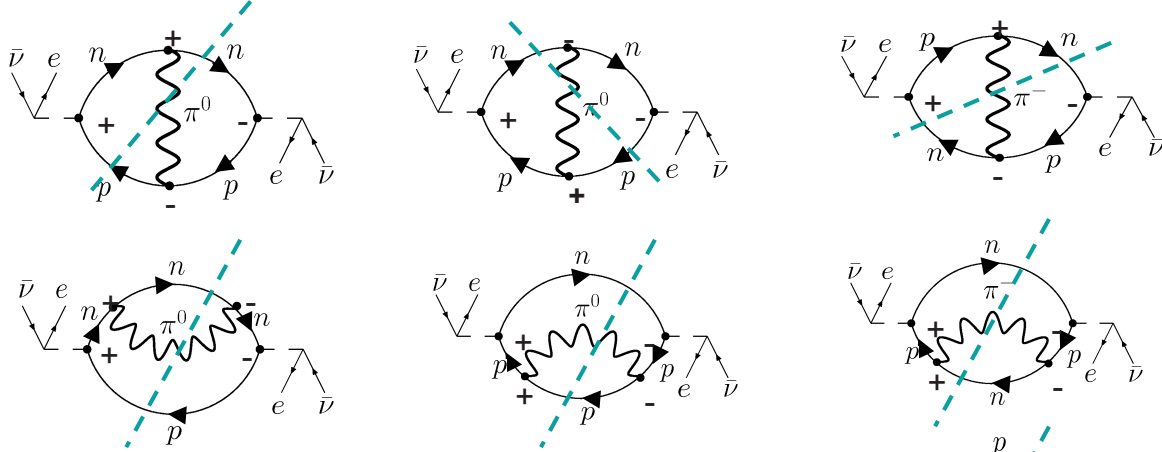

thick pion line (here up to $2^{\text {nd }}$ order):
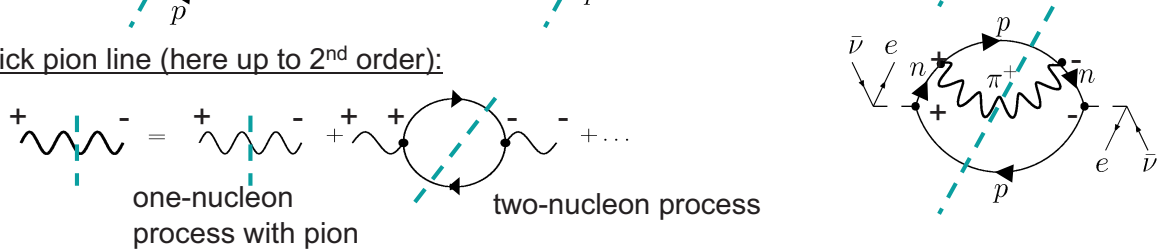

\section{Optical theorem for modified URCA reactions}

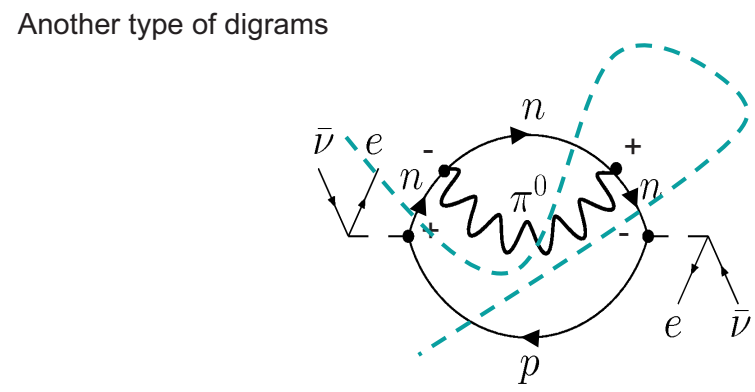

which can be cut in more than two pieces vanish in quasi-particle approximations for fermions 


\section{Optical theorem for modified URCA reactions}

One and two-nucleon processes are treated on equal footing

$\checkmark$ Exchange diagrams are automatically included

$\checkmark$ Both $n n \rightarrow n p e \bar{\nu}$ and $n p \rightarrow p p e \bar{\nu}$ reactions are treated on equal footing

$\checkmark$ We need second-order diagram in pion propagator (!)

since $f_{\pi N N} m_{\pi} \simeq 1$ (not $\left.\ll 1\right)$, in general we should include all diagrams?

$\Rightarrow$ We can treat fermions in QPA (for $T \ll E_{\mathrm{F}}$ ) but (!)

intermediate bosons should be treated beyond QPA, including width effects.

\section{Superfluid matter}

In non-superfluid medium $\left(T>T_{c} \sim 0.1-1 \mathrm{MeV}\right)$

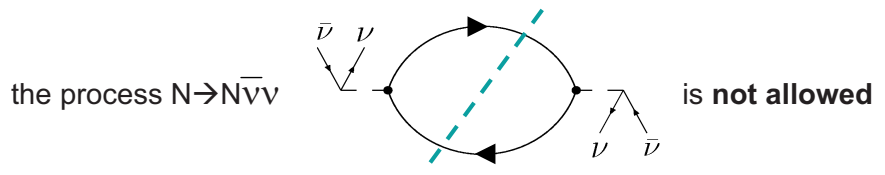

In superfluid medium $\left(T<T_{c}\right)$

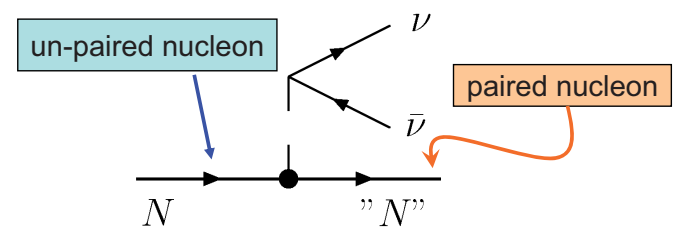

new "quasi"-one-nucleon-like processes become permitted 


\section{Green's function formalism for superfluid matter}

We will use Green's function formalism in non-equilibrium diagram technique

[Senatorov, Voskresensky, Sov J. Nucl. Phys. 45 (1987)]

Besides normal Green's functions one introduces anomalous nucleon Green's functions on the Schwinger-Keldysh contour:

$$
\begin{aligned}
& i \widehat{F}^{(1)}(x, y)={ }^{a} \hookrightarrow^{b}=<N\left|\mathcal{T}_{\mathcal{C}} \widehat{\Psi}^{a}(x) \widehat{\Psi}^{b}(y)\right| N+2> \\
& i \widehat{F}^{(2)}(x, y)={ }_{a} \longleftrightarrow_{b}=<N+2\left|\mathcal{T}_{\mathcal{C}} \widehat{\Psi}_{a}^{\dagger}(x) \widehat{\Psi}_{b}^{\dagger}(y)\right| N>
\end{aligned}
$$

$F^{(1,2)}$ are matrices in spin space, $a$ and $b$ are spin indices

$$
\widehat{F}^{(1) a b}=F g^{a b}, \quad \widehat{F}_{a b}^{(2)}=F g_{a b}
$$

$$
g^{a b}=-g^{b a} \quad g^{12}=1
$$

\section{Green's function formalism for superfluid matter}

- vertices We can have two types of diagram now:

with normal GFs

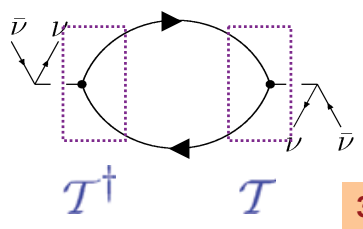

with anomalous GFs
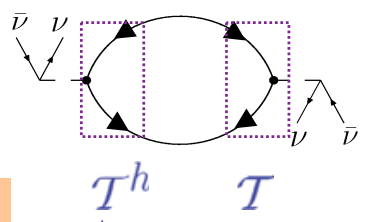

additional vertex for "hole"

$$
\mathcal{T}^{h}(p, q)=\sigma_{2} \mathcal{T}^{T}(-p,-q) \sigma_{2}
$$

For vector vertex:

$$
\mathcal{T}_{V}^{\mu}=\left(\mathcal{T}_{V}^{0}, \mathcal{T}_{V}\right) \longrightarrow\left[\mathcal{T}_{V}^{\mu}\right]^{h}=\left(\mathcal{T}_{V}^{0},-\mathcal{T}_{V}\right)
$$

For axial-vector vertex:

$$
\mathcal{T}_{A}^{\mu}=\left(\mathcal{T}_{A}^{0}, \mathcal{T}_{A}\right) \longrightarrow\left[\mathcal{T}_{A}^{\mu}\right]^{h}=\left(\mathcal{T}_{A}^{0},-\mathcal{T}_{A}\right)
$$




\section{Green's function formalism for superfluid matter}

$$
\begin{gathered}
\left(G^{-+}\right)_{b}^{a}=2 \pi i f(\mathrm{E})\left[u_{p}^{2} \delta(E-E(p))+v_{p}^{2} \delta(E+E(p))\right] \delta_{b}^{a} \\
\left(F^{-+}\right)_{a b}=-2 \pi i f(\mathrm{E}) u_{p} v_{p}[\delta(E-E(p))-\delta(E+E(p))] g_{a b} \\
u_{p}^{2}=\frac{E_{p}+\epsilon_{p}}{2 E_{p}}, \quad v_{p}^{2}=\frac{E_{p}-\epsilon_{p}}{2 E_{p}} \\
E_{p}^{2}=\epsilon_{p}^{2}+\Delta^{2}(p), \quad \epsilon_{p}=v_{\mathrm{F}}\left(p-p_{\mathrm{F}}\right)
\end{gathered}
$$

For $v_{p} \rightarrow 0 \Rightarrow$ standard "normal matter" case.

Terms $\propto v_{p}$ are $\propto \Delta \Rightarrow G$ and $F$ are now not free but QP Green's functions (!)

\section{Probability of the PBF processes ( $1 S_{0}$ pairing)}

$$
\begin{aligned}
& V \rightarrow+\lambda_{\nu}^{\nu} \\
& -i \Pi_{0}^{-+}=\frac{G^{2}}{2} \operatorname{Tr}\left\{l^{\nu} l^{\dagger \mu}\right\}\left(-i \Pi_{0, \mu \nu}^{-+}[\text {nucl }]\right) \\
& -i \Pi_{0, \mu \nu}^{-+}[\mathrm{nucl}]=\int \frac{d^{4} p}{(2 \pi)^{4}}(-1)\left[(-i)\left(\eta_{v} J_{\mu}^{V}-J_{\mu}^{A}\right) i G^{-+} i\left(\eta_{v} J_{\nu}^{V}-J_{\nu}^{A}\right) i G^{+-}\right.
\end{aligned}
$$

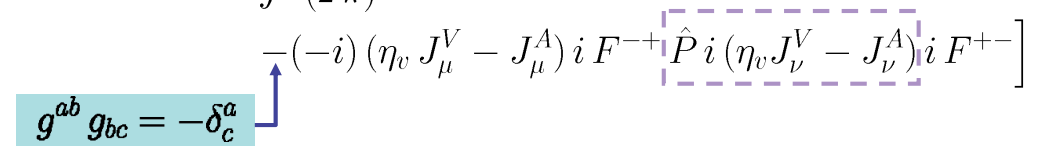

Here $\eta_{v}=1$ for $n n$ pairing and $\eta_{v}=c_{v}$ for $p p$.

The probability of the nPBF (pPBF) processes

$$
\begin{aligned}
-i \Pi_{0}^{-+} & =-i \Pi_{0, N}^{-+}-i \Pi_{0, A}^{-+} \\
& \simeq 2 G^{2} \int \frac{d^{4} p}{(2 \pi)^{4}} \omega_{1} \omega_{2}\left[\left(\eta_{v}^{2}+3 g_{A}^{2}\right) G^{-+} G^{+-}-\left(\eta_{v}^{2}-3 g_{A}^{2}\right) F^{-+} F^{+-}\right]
\end{aligned}
$$




\section{Probability of the PBF processes ( $1 S_{0}$ pairing)}

The first (normal) contribution contains four terms.

Three of them yield zero due to energy conservations.

$-i \Pi_{0, N}^{-+}=\frac{2 G^{2} \omega_{1} \omega_{2}}{(2 \pi)^{2}}\left(\eta_{v}^{2}+3 g_{A}^{2}\right) \int d^{3} p \frac{u_{p+q}^{2} v_{p}^{2} \delta\left(\omega-E_{p}-E_{\boldsymbol{p}+\boldsymbol{q}}\right)}{\left(\exp \left[\left(\omega-E_{\boldsymbol{p}}\right) / T\right]+1\right)\left(\exp \left[\left(E_{\boldsymbol{p}}\right) / T\right]+1\right)}$

Introducing $\boldsymbol{p}_{1}=\boldsymbol{p}, \boldsymbol{p}_{2}=\boldsymbol{p}+\boldsymbol{q}$ we rewrite

$$
\int d^{3} p=2 \pi \int_{0}^{\infty} p_{1} d p_{1} \frac{1}{q} \int_{p_{1}-q}^{p_{1}+q} p_{2} d p_{2}
$$

Assuming $v_{F}^{2} \ll 1$, we may put $E_{p_{2}} \simeq E_{p_{1}}$.

$$
-i \Pi_{0, N}^{-+}=\frac{G^{2} \omega_{1} \omega_{2} \Delta^{2}}{\omega^{2}}\left(\eta_{v}^{2}+3 g_{A}^{2}\right) \frac{\tilde{p}^{2}}{\left(d E_{p} / d p\right)_{\tilde{p}}(\exp [\omega /(2 T)]+1)^{2}} \theta(\omega-2 \Delta),
$$

where $\tilde{p}$ is the root of the relation $2 E_{p}=\omega$, and $\left(d E_{p} / d p\right)_{\tilde{p}}=v_{\mathrm{F}} \frac{\sqrt{\omega^{2}-4 \Delta^{2}}}{\omega}$.

\section{Emissivity of the PBF processes ( $1 S_{0}$ pairing)}

The anomalous term is $\propto u_{p+q}^{2} v_{p}^{2}$ :

$$
-i \Pi_{0, A}^{-+}=\frac{2 G^{2} \omega_{1} \omega_{2}}{(2 \pi)^{2}}\left(\eta_{v}^{2}-3 g_{A}^{2}\right) \int d^{3} p \frac{u_{p+q}^{2} v_{p}^{2} \delta\left(\omega-E_{p}-E_{\boldsymbol{p}+\boldsymbol{q}}\right)}{\left(\exp \left[\left(\omega-E_{\boldsymbol{p}}\right) / T\right]+1\right)\left(\exp \left[\left(E_{\boldsymbol{p}}\right) / T\right]+1\right)}
$$

Thus one obtains

$$
-i \Pi_{0, A}^{-+}=\frac{2 m_{N} p_{\mathrm{F}, N} G^{2} \omega_{1} \omega_{2} \Delta^{2}}{\pi \omega \sqrt{\omega^{2}-4 \Delta^{2}}}\left(\eta_{v}^{2}-3 g_{A}^{2}\right) \frac{\theta(\omega-2 \Delta)}{(\exp [\omega /(2 T)]+1)^{2}}
$$

\section{Only vector term in $\Pi_{0, N}^{-+}+\Pi_{0, A}^{-+}$remains.}

For the emissivity of the $\mathrm{nPFB}$ reaction we find

$$
\epsilon_{\nu}\left(1 S_{0}\right)=\epsilon_{\nu}^{\mathrm{vect}}\left(1 S_{0}\right)+\epsilon_{\nu}^{\mathrm{axial}}\left(1 S_{0}\right)=N_{\nu} \frac{4}{15} \frac{G^{2} m_{N} p_{\mathrm{F}, N} \Delta^{7}}{\pi^{5}} I(\Delta / T) \times \eta_{v}^{2},
$$

$N_{\nu}$ is the number of neutrino species. 


\section{Emissivity of the PBF processes ( $1 S_{0}$ pairing)}

$$
\begin{gathered}
I(x)=\int_{0}^{\infty} \operatorname{ch} y d y /(\exp [x \operatorname{ch} y]+1)^{2}, \\
I(x \gg 1)=\exp (-2 x) \sqrt{\pi /(4 x)}, \quad I(x \ll 1) \simeq 0.6 x^{-5}
\end{gathered}
$$

$\checkmark$ Note that $\epsilon_{\nu}(x \gg 1) \propto \Delta^{7} \exp (-2 \Delta / T)$, [Senatorov, Voskresensky, Sov. Nucl. Phys. 45 (1987)], rather than to $T^{7} \exp (-2 \Delta / T)$, as it is often claimed.

For $T_{9}=1$ then would be $\epsilon_{\nu}^{\mathrm{PBF}} \sim 0.1 \epsilon_{\nu}^{\mathrm{MU}}$, whereas with correct asymptotic for $\Delta=0.5 \mathrm{MeV}, T_{9}=1$ one gets extra $10^{7}$.

$\checkmark c_{V}^{2} \simeq 0.006$ suppression factor for pPBF but $\Pi_{\mu \nu}^{-+}$with bare vertices and QP Green's functions docs not fulfill vector current conservation (!)

dressing of vertices is required.

\section{Full Green's function}

particle-line

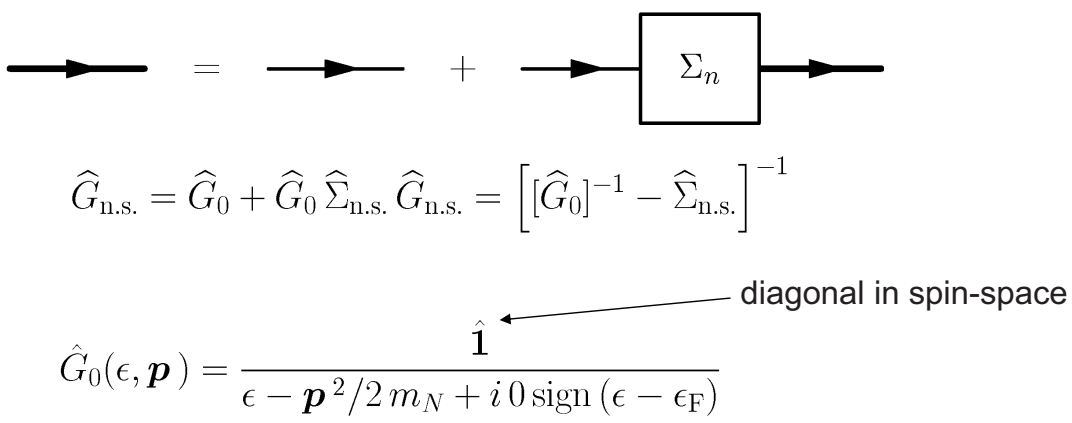

analogously for the hole-line

$$
\Sigma_{n}
$$

\section{particle-particle, particle-hole hole-hole interactions}




\section{Particle-particle interaction}
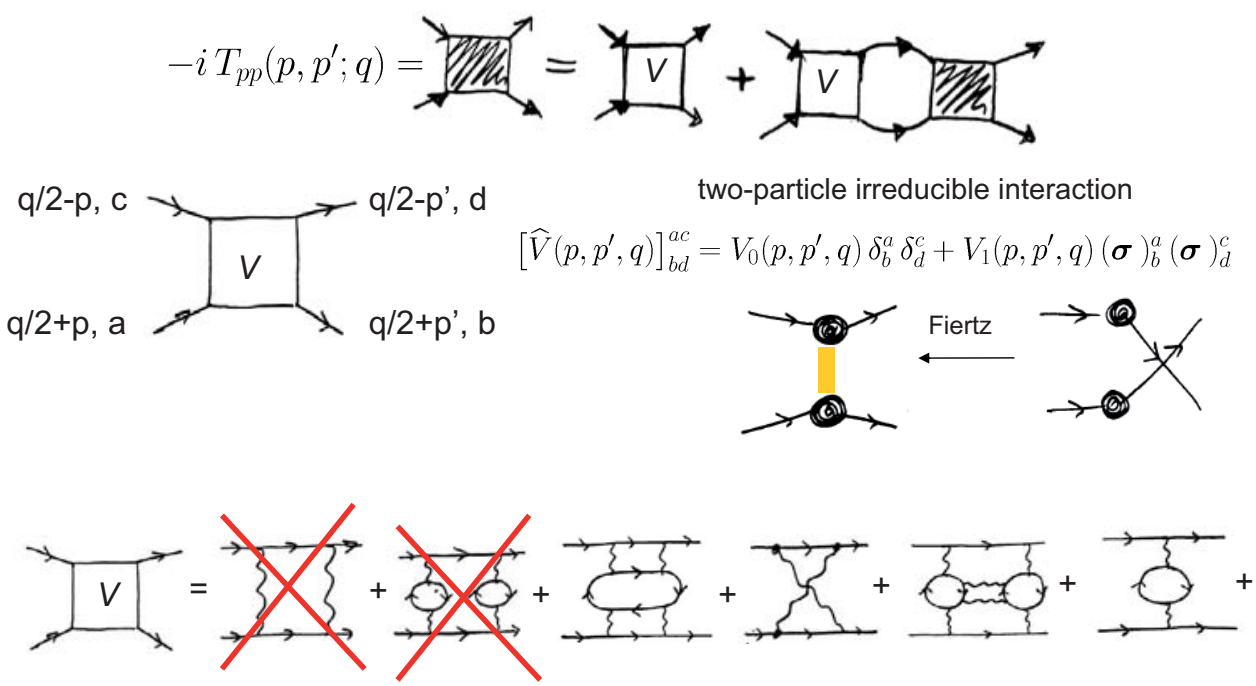

$$
\widehat{T}_{\mathrm{pp}}\left(p, p^{\prime}, q\right)=\widehat{V}\left(p, p^{\prime}, q\right)+\int \frac{\mathrm{d}^{4} p^{\prime \prime}}{(2 \pi)^{4} i} \widehat{V}\left(p, p^{\prime \prime}, q\right) \widehat{G}\left(q / 2+p^{\prime \prime}\right) \widehat{G}\left(q / 2-p^{\prime \prime}\right) \widehat{T}_{\mathrm{pp}}\left(p^{\prime \prime}, p^{\prime}, q\right)
$$

\section{Particle-particle interaction}
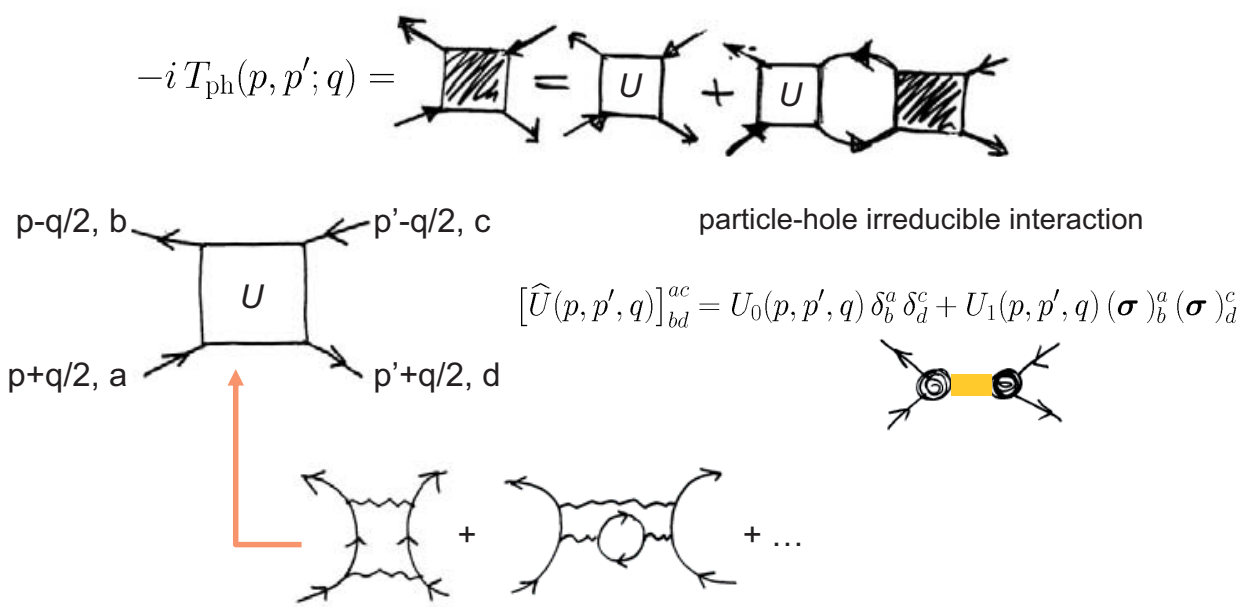

$\widehat{T}_{\mathrm{ph}}\left(p, p^{\prime}, q\right)=\widehat{U}\left(p, p^{\prime}, q\right)+\int \frac{\mathrm{d}^{4} p^{\prime \prime}}{(2 \pi)^{4} i} \widehat{U}\left(p, p^{\prime \prime}, q\right) \widehat{G}\left(q / 2+p^{\prime \prime}\right) \widehat{G}^{h}\left(q / 2-p^{\prime \prime}\right) \widehat{T}_{\mathrm{ph}}\left(p^{\prime \prime}, p^{\prime}, q\right)$ 


\section{"Charge" of particle and hole}

Coupling of the external field to a particle

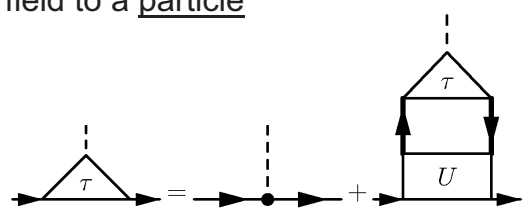

and a hole

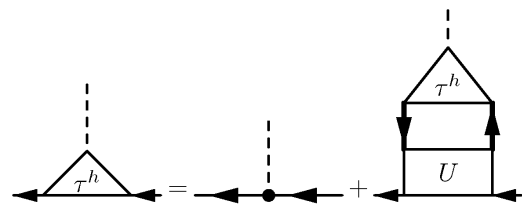

- one-nucleon reaction
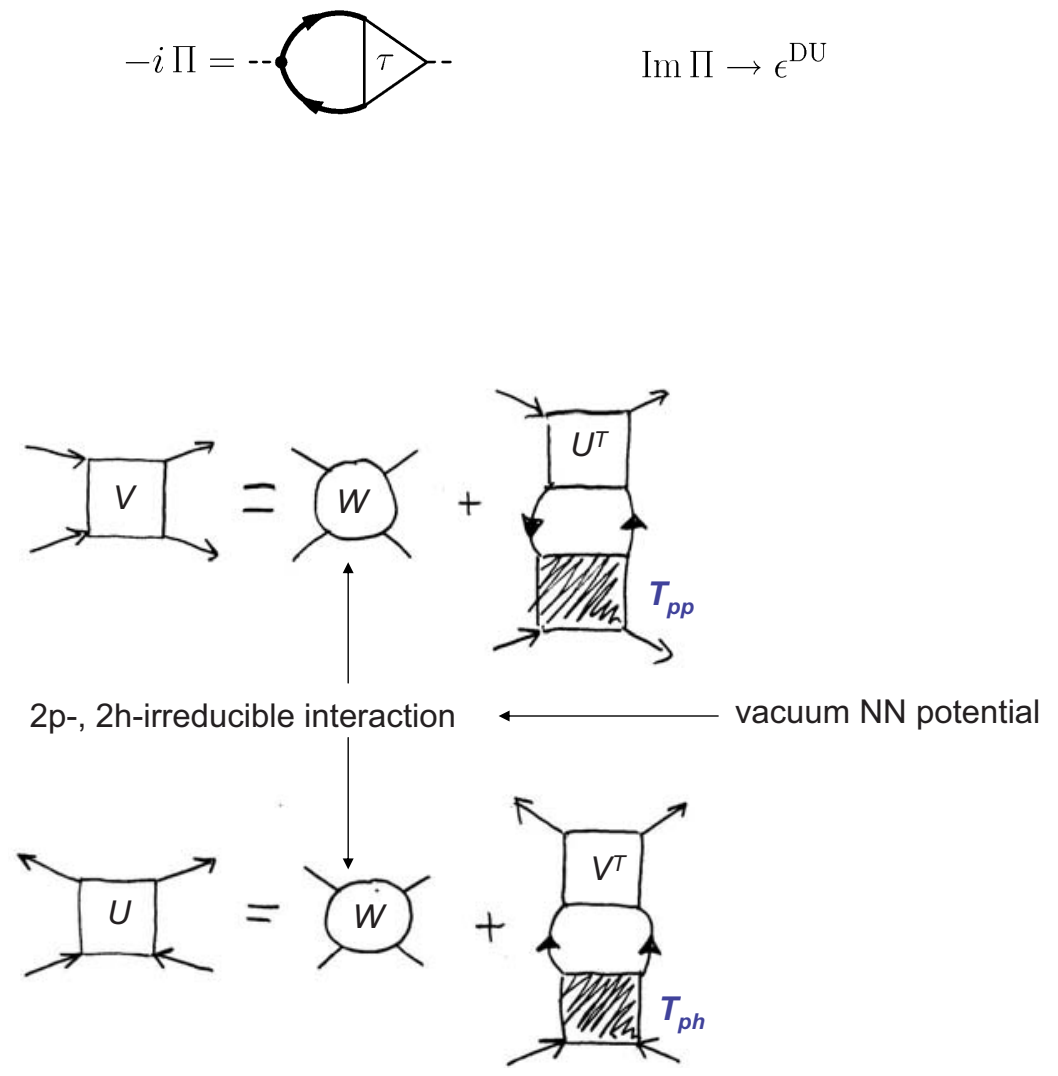

[Wambach, Ainsworth,Pines NPA (1993)] 


\section{Vector current conservation}

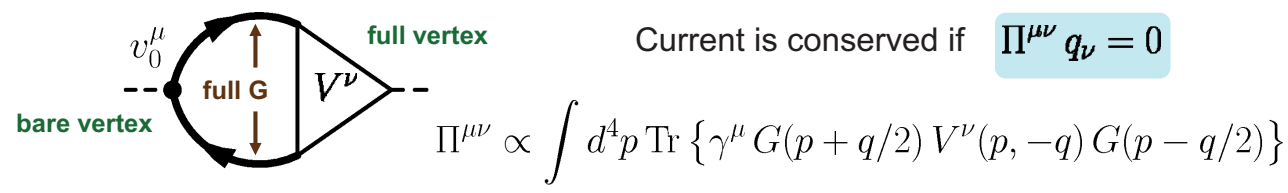

If the relation

$$
q_{\mu} V^{\mu}(p, q)=G^{-1}(p+q / 2)-G^{-1}(p-q / 2) \quad \text { Is fulfilled }
$$

$$
\Pi^{\mu \nu} q^{\nu} \propto \int d^{4} p \operatorname{Tr}\left\{\gamma^{\mu}[G(p-q / 2)-G(p+q / 2)]\right\}=0
$$

The Ward identities impose non-trivial relations between vertex functions and Green's functions, which synchronize any modification of the Green's function with a corresponding change in the vertex function.

in non-relativistic limit for free $\mathrm{G}$ and vertices: $\quad \tau_{0}^{\mu}=(1, \boldsymbol{v}) \quad G(p)=\left(\epsilon-p^{2} / 2 m\right)^{-1}$

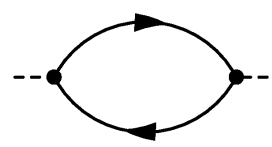

$$
q \cdot \tau_{0}=\omega-\boldsymbol{v} \boldsymbol{q} \equiv G_{0}^{-1}(p+q / 2)-G_{0}^{-1}(p-q / 2)
$$

The Ward identity is fulfilled and the current is conserved

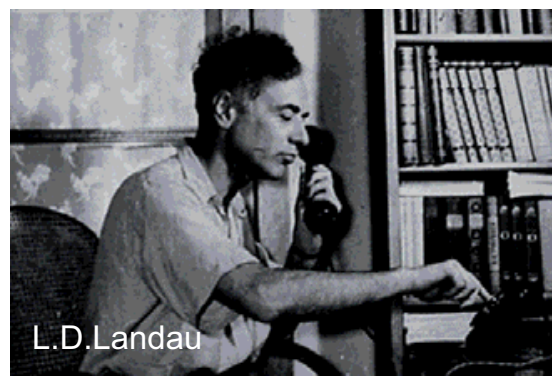

NUCLEAR FERMI LIQUID

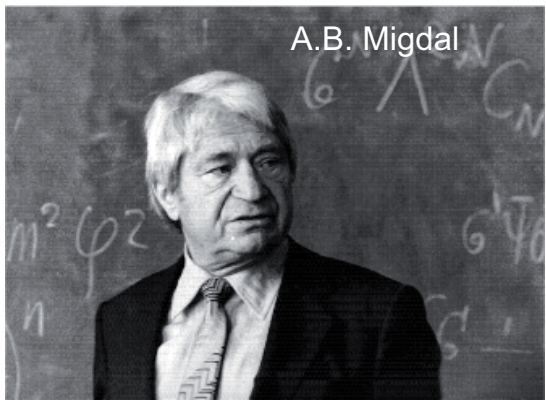




\section{Landau Fermi liquid approach}

\section{interacting fermions}

\section{system of quasi-particles}

\section{quantized excitations in the system}

quasi-particles $\neq$ original "bare" fermions [constituents of the system]

Landau wrote the Boltzmann eq.

for q.p distribution function: $n(\boldsymbol{x}, \boldsymbol{p}, t)$

$$
\frac{d n}{d t}=\frac{\partial n}{\partial t}+\dot{\boldsymbol{x}} \frac{\partial n}{\partial \boldsymbol{x}}+\dot{\boldsymbol{p}} \frac{\partial n}{\partial \boldsymbol{p}}=I(n)
$$

equations of motion for q.p.

$$
\dot{\boldsymbol{x}}=\frac{\partial \bar{e}(\boldsymbol{p}, \boldsymbol{x})}{\partial \boldsymbol{p}}, \quad \dot{\boldsymbol{p}}=-\frac{\partial \bar{e}(\boldsymbol{p}, \boldsymbol{x})}{\partial \boldsymbol{x}}
$$

"generalized" velocity Newton's law

$$
\frac{\partial n}{\partial t}+\frac{\partial \bar{e}(\boldsymbol{p}, \boldsymbol{x})}{\partial \boldsymbol{p}} \frac{\partial n}{\partial \boldsymbol{x}}-\frac{\partial \bar{e}(\boldsymbol{p}, \boldsymbol{x})}{\partial \boldsymbol{x}} \frac{\partial n}{\partial \boldsymbol{p}}=I(n)
$$

$\mathcal{F}=\int \boldsymbol{p} n \frac{d^{3} p}{(2 \pi)^{3}} \quad \frac{\partial \mathcal{F}}{\partial t}=\int \boldsymbol{p} \frac{\partial n}{\partial t} \frac{d^{3} p}{(2 \pi)^{3}}=\int \boldsymbol{p} I(n) \frac{d^{3} p}{(2 \pi)^{3}}-\int \boldsymbol{p}\left[\frac{\partial \bar{e}}{\partial \boldsymbol{p}} \frac{\partial n}{\partial \boldsymbol{x}}-\frac{\partial \bar{e}}{\partial \boldsymbol{x}} \frac{\partial n}{\partial \boldsymbol{p}}\right] \frac{d^{3} p}{(2 \pi)^{3}}$ momentum flux density

$$
\begin{aligned}
& =-\frac{\partial}{\partial x_{j}} \int \boldsymbol{p} n \frac{\partial \bar{e}}{\partial \boldsymbol{p}_{j}} \frac{d^{3} p}{(2 \pi)^{3}}-\int n \frac{\partial \bar{e}}{\partial \boldsymbol{x}} \frac{d^{3} p}{(2 \pi)^{3}} \\
& =\frac{\partial}{\partial x_{j}} \Pi^{j}+\int \bar{e} \frac{\partial n}{\partial \boldsymbol{x}} \frac{d^{3} p}{(2 \pi)^{3}}
\end{aligned}
$$

momentum conservation $\quad 0=\int \mathcal{F} d^{3} x=\int \frac{\partial}{\partial x_{j}} \Pi^{j} d^{3} x+\int d^{3} x \int \bar{e} \frac{\partial n}{\partial \boldsymbol{x}} \frac{d^{3} p}{(2 \pi)^{3}}$

$$
\begin{gathered}
\Rightarrow \int d^{3} x \int \bar{e} \frac{\partial n}{\partial \boldsymbol{x}} \frac{d^{3} p}{(2 \pi)^{3}}=0 \\
\int \bar{e} \frac{\downarrow}{\partial \boldsymbol{x}} \frac{d^{3} p}{(2 \pi)^{3}}=\frac{\partial}{\partial \boldsymbol{x}} E \\
\delta E=\int \bar{e} \delta n \frac{d^{3} p}{(2 \pi)^{3}} \\
\frac{\delta E}{\delta n(p)}=\bar{e}(p)
\end{gathered}
$$




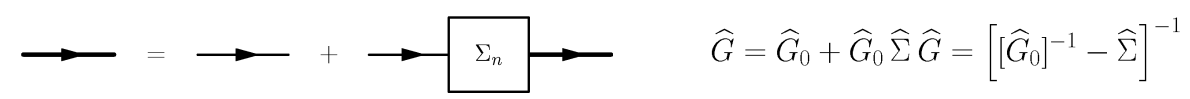

$$
\begin{aligned}
& T \ll \varepsilon_{\mathrm{F}, n}, \varepsilon_{\mathrm{F}, \mathrm{p}} \text { and } \epsilon \sim \epsilon_{\mathrm{F}}, p \sim p_{\mathrm{F}}\left\lceil\text { pole residue } a^{-1}=1-\left.\frac{\partial}{\partial \epsilon} \Sigma(\epsilon, 0, T)\right|_{\epsilon \simeq \epsilon_{\mathrm{F}}}\right. \\
& G(\epsilon, \boldsymbol{p})=\frac{a}{\epsilon-\epsilon_{p}+i \gamma \epsilon^{2} \operatorname{sign} \epsilon}+G_{\mathrm{reg}}(\epsilon, \boldsymbol{p}) \\
& \text { q.p. energy } \\
& \text { q.p. width } \\
& \epsilon_{p}=\frac{p^{2}-2 m_{N} \mu_{N}}{2 m_{N}^{*}} \approx \frac{p^{2}-p_{\mathrm{F}}^{2}}{2 m_{N}^{*}} \approx v_{\mathrm{F}}\left(p-p_{\mathrm{F}}\right) \\
& \text { q.p. effective mass } \downarrow \\
& \frac{1}{m_{N}^{*}}=\frac{a}{m_{N}}+\left.2 a \frac{\partial}{\partial p^{2}} \Sigma(\epsilon, \boldsymbol{p}, T)\right|_{p=0, \epsilon \simeq \epsilon_{\mathrm{F}}} \quad G_{\mathrm{reg}}(\epsilon, \boldsymbol{p}) \text { complicated background part }
\end{aligned}
$$

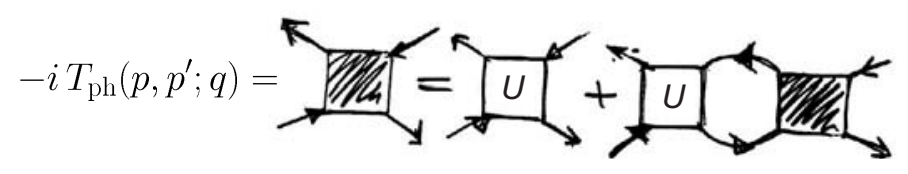

$$
\begin{aligned}
& \widehat{T}_{\mathrm{ph}}\left(p, p^{\prime}, q\right)=\widehat{U}\left(p, p^{\prime}, q\right)+\int \frac{\mathrm{d}^{4} p^{\prime \prime}}{(2 \pi)^{4} i} \widehat{U}\left(p, p^{\prime \prime}, q\right) \widehat{G}\left(q / 2+p^{\prime \prime}\right) \widehat{G}^{h}\left(q / 2-p^{\prime \prime}\right) \widehat{T}_{\mathrm{ph}}\left(p^{\prime \prime}, p^{\prime}, q\right) \\
& \gamma=-\lim _{\epsilon \rightarrow 0} \operatorname{Im} \Sigma^{R}\left(\epsilon, p^{2}=2 m_{N} \mu_{N}, T\right) / \epsilon^{2} \\
& \text { small for } T<<\varepsilon_{F}
\end{aligned}
$$

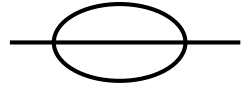$$
G(q / 2+p) G^{h}(q / 2-p)=G(q / 2+p) G(p-q / 2)
$$$$
=\frac{a}{\left[\epsilon+\omega / 2-\epsilon_{\boldsymbol{p}+\boldsymbol{q} / 2}+i 0 \operatorname{sign}(\epsilon+\omega / 2)\right]} \frac{a}{\left[\epsilon-\omega / 2-\epsilon_{\boldsymbol{p}-\boldsymbol{q} / 2}+i 0 \operatorname{sign}(\epsilon-\omega / 2)\right]}+\tilde{B}(p, q)
$$$$
\simeq a^{2} \delta(\epsilon) \int d \epsilon \frac{1}{\left[\epsilon+\omega / 2-\epsilon_{\boldsymbol{p}+\boldsymbol{q} / 2}+i 0 \operatorname{sign}(\epsilon+\omega / 2)\right]} \frac{1}{\left[\epsilon-\omega / 2-\epsilon_{\boldsymbol{p}-\boldsymbol{q} / 2}+i 0 \operatorname{sign}(\epsilon-\omega / 2)\right]}+B(p, q)
$$$$
=-2 \pi i a^{2} \delta(\epsilon) \frac{f(\boldsymbol{p}+\boldsymbol{q} / 2)-f(\boldsymbol{p}-\boldsymbol{q} / 2)}{\omega-\epsilon_{\boldsymbol{p}+\boldsymbol{q} / 2}+\epsilon_{\boldsymbol{p}-\boldsymbol{q} / 2}+i 0}+B(p, q)
$$

for $q \rightarrow 0$

$$
\begin{array}{r}
G(q / 2+p) G^{h}(q / 2-p) \simeq 2 \pi i a^{2} \delta(\epsilon) \frac{v_{\mathrm{F}} \boldsymbol{q} \boldsymbol{n}}{\omega-v_{\mathrm{F}} \boldsymbol{q} \boldsymbol{n}+i 0} \delta\left(p-p_{\mathrm{F}}\right)+B(p, q) \\
\boldsymbol{n}=\boldsymbol{p} / p
\end{array}
$$




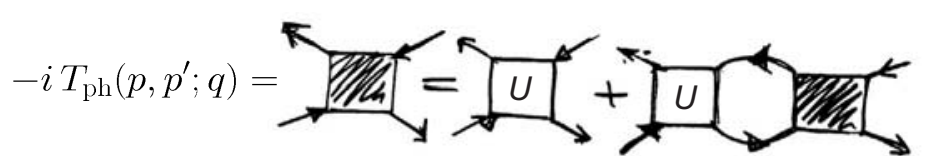

for $|\boldsymbol{p}| \simeq p_{\mathrm{F}} \simeq\left|\boldsymbol{p}^{\prime}\right|$ and $|\boldsymbol{q} \boldsymbol{p}|<<\omega<<\epsilon_{\mathrm{F}}$

$$
\begin{array}{r}
\widehat{T}_{\mathrm{ph}}\left(\boldsymbol{n}, \boldsymbol{n}^{\prime}, q\right)=\widehat{\Gamma}^{\omega}\left(\boldsymbol{n}, \boldsymbol{n}^{\prime}\right)-\int \frac{d \Omega_{p^{\prime \prime}}}{4 \pi} \widehat{\Gamma}^{\omega}\left(\boldsymbol{n}, \boldsymbol{n}^{\prime}\right) A(\boldsymbol{n}, q) \widehat{T}_{\mathrm{ph}}\left(\boldsymbol{n}, \boldsymbol{n}^{\prime}, q\right) \\
\Lambda(\boldsymbol{n}, q)=a^{2} \frac{m^{*} p_{\mathrm{F}}}{\pi^{2}} \frac{v_{\mathrm{F}} \boldsymbol{q} \boldsymbol{n}}{\omega-v_{\mathrm{F}} \boldsymbol{q} \boldsymbol{n}+i 0}
\end{array}
$$

complicated dynamics is here:

$$
\widehat{\Gamma}_{\mathrm{ph}}^{\omega}\left(\boldsymbol{n}, \boldsymbol{n}^{\prime}\right)=\widehat{U}\left(\boldsymbol{n}, \boldsymbol{n}^{\prime}\right)-\int \frac{d^{4} p^{\prime \prime}}{(2 \pi)^{4} i} \widehat{U}\left(\boldsymbol{n}, \boldsymbol{n}^{\prime}\right) B(p, q=0) \widehat{\Gamma}_{\mathrm{ph}}^{\omega}\left(\boldsymbol{n}, \boldsymbol{n}^{\prime}\right)
$$

\section{parameterize}

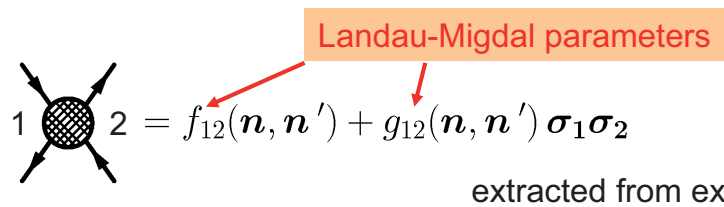

extracted from experiment

$$
\begin{aligned}
& 1 \\
& =C_{0}\left(f+f^{\prime} \boldsymbol{\tau}_{1} \boldsymbol{\tau}_{2}+g \boldsymbol{\sigma}_{1} \boldsymbol{\sigma}_{2}+g^{\prime} \boldsymbol{\sigma}_{1} \boldsymbol{\sigma}_{2} \boldsymbol{\tau}_{1} \boldsymbol{\tau}_{2}\right) \\
& C_{0}=\frac{\pi^{2}}{m_{N} p_{F N}\left(n_{0}\right)} \simeq 300 \mathrm{MeV} \mathrm{fm}{ }^{3} \simeq 0.77 m_{\pi}^{-2} \text { introduced for convenience } \\
& f\left(\boldsymbol{n}, \boldsymbol{n}^{\prime}\right)=\sum_{l} f_{l} P_{l}\left(\cos \theta_{\boldsymbol{n} \boldsymbol{n}^{\prime}}\right) \quad g\left(\boldsymbol{n}, \boldsymbol{n}^{\prime}\right)=\sum_{l} g_{l} P_{l}\left(\cos \theta_{\boldsymbol{n} \boldsymbol{n}^{\prime}}\right) \\
& \text { to be fitted to empirical information (nucleus properties) } \\
& \text { effective mass } \quad m^{*}=m\left(1+\frac{2}{3} f_{1}\right) \\
& \text { compressibility } \quad K=6 \frac{p_{\mathrm{F}}^{2}}{m^{*}}\left(1+2 f_{0}\right) \\
& \text { symmetry energy } E_{\mathrm{sym}}=\frac{1}{3} \frac{p_{\mathrm{F}}^{2}}{2 m^{*}}\left(1+2 f_{0}^{\prime}\right)
\end{aligned}
$$

[Saperstein, Fayans, et al. 1995, 1998]

$f \simeq 0, f^{\prime} \simeq 0.5-0.6, g \simeq 0.05 \pm 0.1, g^{\prime} \simeq 1.1 \pm 0.1$ 


\section{Nuclear Fermi liquid. Approximations}

- Quasiparticle approximation for nucleons, $T \ll \epsilon_{\mathrm{FN}}$.

Only in this case diagrams with open nucleon lines make sense.

Otherwise the quasiparticle width is not negligible

and closed diagram technique has to be used.

- Reduction of the more local interaction to the point-like interaction

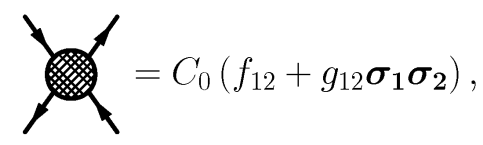

Assumption that the Landau-Migdal parameters, $f_{12}, g_{12}$, are constants Is a rough approximation.

- explicit pionic degrees of freedom

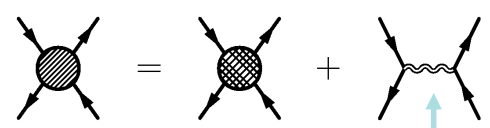

pion with residual (irreducible in $\mathrm{NN}^{-1}$ and $\Delta \mathrm{N}^{-1}$ ) S-wave $\pi \mathrm{N}$ interaction and $\pi \pi$ scattering "

- explicit $\Delta$ degrees of freedom

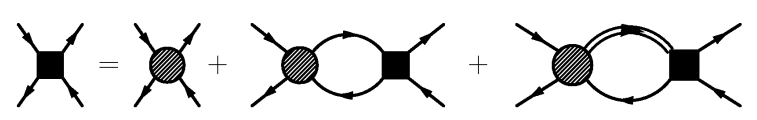

Part of the interaction involving $\Delta$ isobar is analogously constructed:

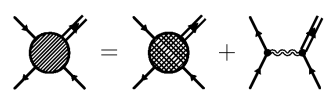




\section{Nuclear Fermi liquid: resummed $N N$ interaction}

Graphically, the resummation is straightforward and yields:

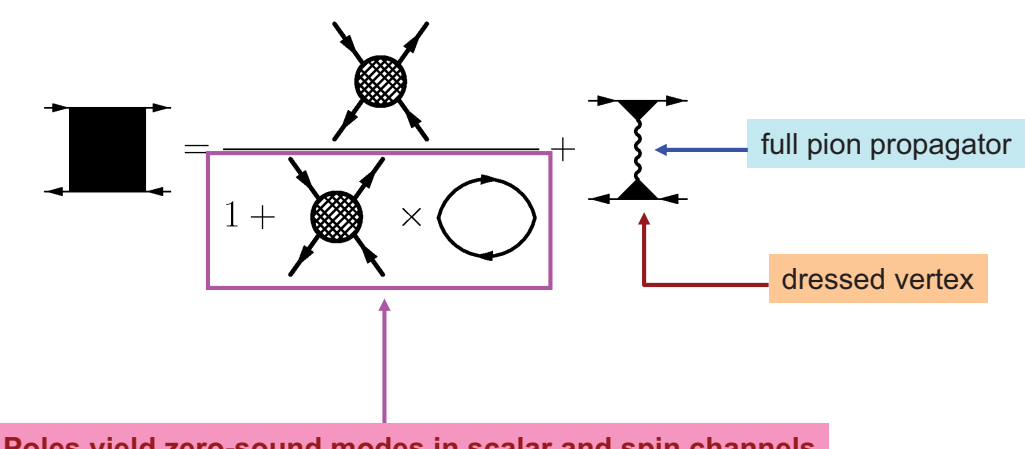

Poles yield zero-sound modes in scalar and spin channels

\section{Nuclear Fermi liquid: effective coupling}

Coupling of the external field to a particle

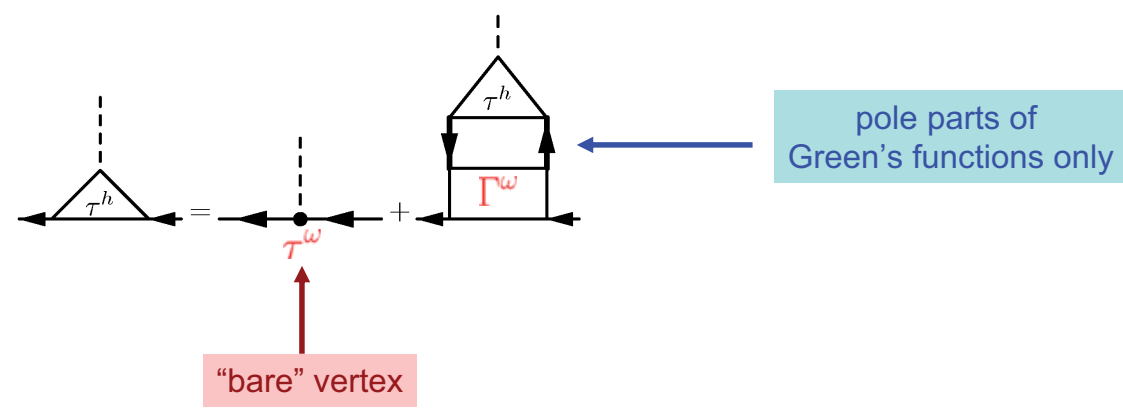


- "Bare" vertices

"bare" vertex after the Fermi-liquid renormalization $\quad \tau_{a}^{\omega}=\left[1+\Gamma_{0}^{\omega}\left(G_{+} G_{-}\right)^{\omega}\right] \tau_{a}^{0}$

$V_{\mu}^{n n} \approx g_{V} \chi_{p}^{\dagger}\left(p^{\prime}\right)(1, v) \chi_{n}(p)$
$A_{\mu}^{n n} \approx g_{A} \chi_{p}^{\dagger}\left(p^{\prime}\right)(\boldsymbol{\sigma} \cdot \boldsymbol{v}, \boldsymbol{\sigma}) \chi_{n}(p)$
$\tau_{V}^{0}-\tau_{A}^{0}=\left(V_{\mu}-A_{\mu}\right) l^{\mu} \quad$ weak interactions
$\hat{\tau}_{V}^{\omega}=g_{V}\left(\tau_{V, 0}^{\omega} l_{0}-\tau_{-, 1}^{\omega} \boldsymbol{l}\right) \quad \lim _{\boldsymbol{q} \rightarrow 0} \int \frac{2 \mathrm{~d}^{4} p}{(2 \pi)^{4} i} G_{+} G_{-}$
$\hat{\tau}_{A}^{\omega}=-g_{A}\left(\tau_{A, 1}^{\omega} \boldsymbol{\sigma} l_{0}-\tau_{A, 0}^{\omega} \boldsymbol{\sigma l}\right) \quad \tau_{V, 0}^{\omega}=\frac{e_{V}}{a}, \quad \tau_{V, 1}^{\omega}=\frac{e_{V}}{a} \boldsymbol{v}$
$\tau_{A, 1}=\frac{e_{A}}{a} \boldsymbol{v}$

$e_{A} \quad e_{V}$ effective charges

$$
\begin{gathered}
e_{V}=1 \quad \omega \tau_{V, 0}^{\omega}-\boldsymbol{q} \tau_{V, 1}^{\omega}=G_{.}^{(\text {pole }),-1}(p+q / 2)-G^{(\text {pole }),-1}(p-q / 2) \\
e_{A}=0.8-0.95 \quad \text { experiment: Gamov-Teller transitions in nuclei } \quad g_{A}^{*} \simeq 1
\end{gathered}
$$

\section{Optical theorem for modified URCA reactions}

$$
\epsilon_{\nu}^{\mathrm{MU}}=\int \frac{d^{3} q_{e}\left(1-f_{e}\right)}{2 \omega_{e}(2 \pi)^{3}} \frac{d^{3} q_{\bar{\nu}}}{2 \omega_{\bar{\nu}}(2 \pi)^{3}} \omega_{\bar{\nu}}\left[-i \Pi_{\mathrm{MU}}^{-+}\left(q_{e}+q_{\bar{\nu}}\right)\right]
$$

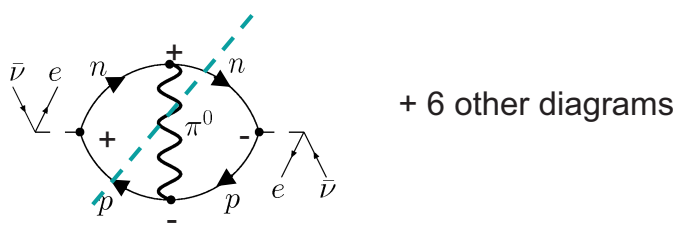

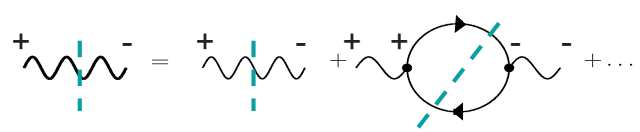


Pion spectrum at order $f_{\pi N N}^{2}$

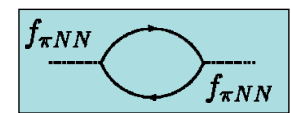

$$
D^{-1}(\omega, k)=\omega^{2}-m_{\pi}^{2}-k^{2}-\Pi_{0}^{R}(\omega, k, n)=0
$$

- Perturbation theory in dimensionless parameter $f_{\pi N N} p_{\mathrm{FN}} \simeq 2$ !

- For $\omega \rightarrow 0, k \sim p_{\mathrm{FN}}$ and for isospin symmetric matter

$$
\begin{gathered}
\Pi_{0}^{R} \simeq-\alpha_{0}-i \beta_{0} \omega, \alpha_{0} \simeq \frac{2 m_{N} p_{F} k^{2} f_{\pi N N}^{2}}{\pi^{2}}>0, \beta_{0} \simeq \frac{m_{N}^{2} k f_{\pi N N}^{2}}{\pi}>0 . \\
\omega \propto-i \omega^{* 2}\left(k_{\min }\right) / \beta_{0} \\
\phi \sim \exp (-i \omega \cdot t) \quad \text { grows with time since } \omega^{* 2}\left(k_{m}\right)=-D^{-1}\left(0, k_{m} \simeq p_{\mathrm{F}}\right)<0 \\
\text { It would happen already at } n>0.3 n_{0}
\end{gathered}
$$

\section{Virtual pion mode}

Dyson equation for the full retarded pion Green's function

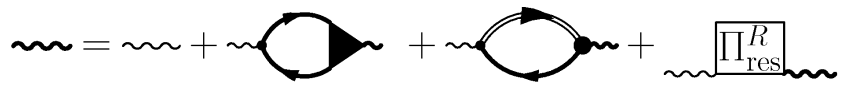

The $\pi \mathrm{N} \Delta$ vertex includes a phenomenological range term.

The full $\pi N N$ vertex takes into account NN short correlations.

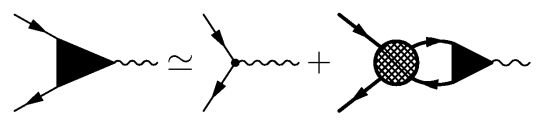

The value of the NN interaction, e.g. in the neutral pion channel, is determined by the full pion propagator at small $\omega$ and $k=p_{F, n}$.

$$
\omega^{* 2}(k)=-\left(D_{\pi}^{R}\right)^{-1}\left(\omega=0, k, \mu_{\pi}\right) .
$$

For $n>n_{c 1}\left(n_{c 1}<n_{0}\right)$ the quantity $\omega^{*}(k)$ has minimum for $k=k_{m} \simeq(0.9-1) p_{\mathrm{Fn}}$.

The quantity $\omega^{*}\left(k_{m}\right)$ has the meaning of the effective pion gap. 


\section{Pion sectra and pion condesation}
$n<n_{c}^{+}$
$n_{c}^{+}<n<n_{c}^{ \pm}$
$n>n_{c}^{ \pm}$
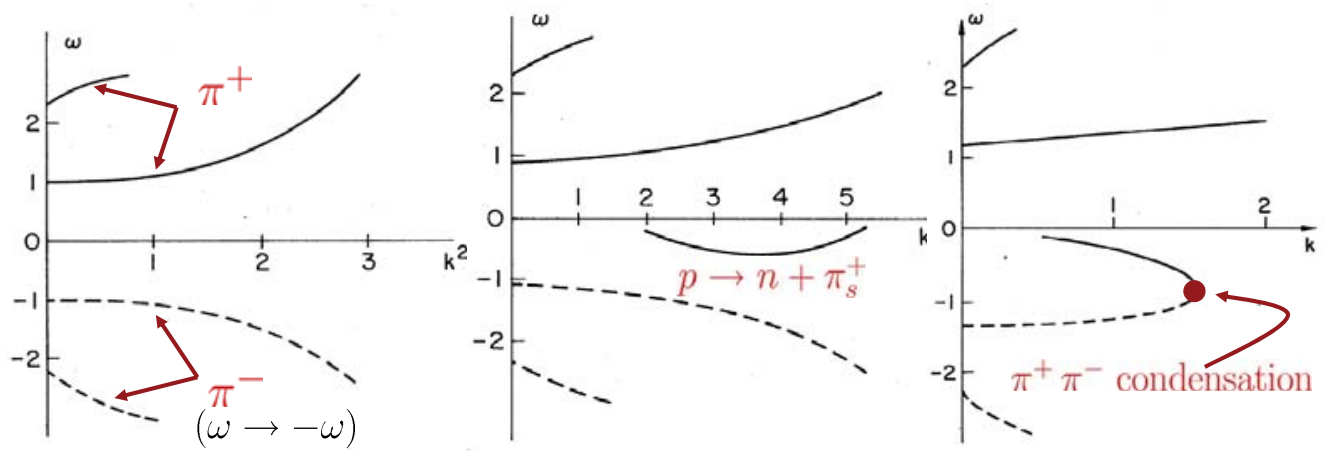

$n_{c}^{+} \lesssim n_{0}, \quad n_{c}^{ \pm} \sim(1-3) n_{0}, \quad n_{c}^{\pi^{0}} \sim(1-3) n_{0} \quad$ [A.B. Migdal, Rev. Mod. Phys. 50 (1978)]

variational calculations [Akmal, Pandharipande, Ravenhall, PRC58 (1998)]:

charged pion condensate: $n_{c} \simeq 2 n_{0} \quad N=Z$

neutral pion condensate: $n_{c} \simeq 2 n_{0} \quad N=Z, \quad n_{c}=1.3 n_{0} \quad N>>Z$

\section{Virtual pion mode}

$\omega^{* 2}(k)=-\left[D_{\pi}^{R}\left(\omega=0, k, \mu_{\pi}\right)\right]^{-1}$

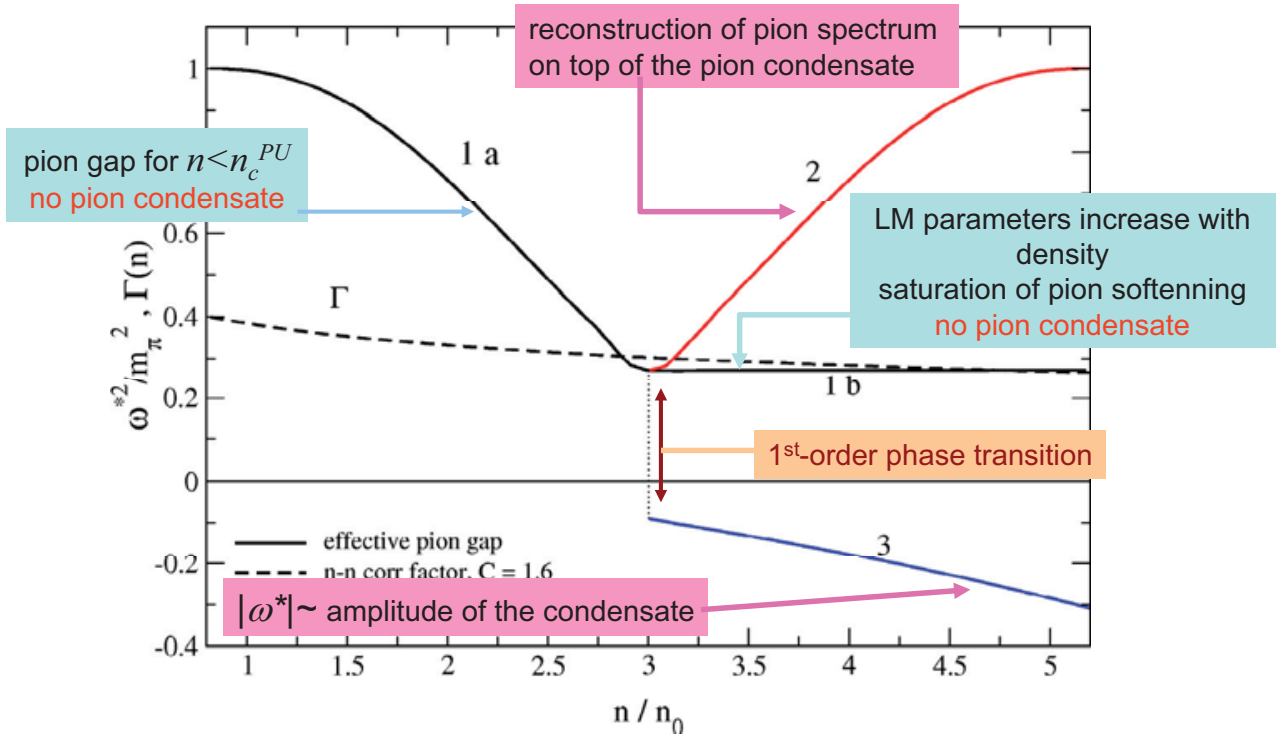




\section{Re-summed $N N$ interaction}

Re-summed NN interaction in neutral channel:

$$
\begin{aligned}
& \Gamma_{\alpha \beta}^{R}=\underbrace{\alpha}_{\alpha}=\frac{C_{0}\left(\mathcal{F}_{\alpha \beta}^{R}+\mathcal{Z}_{\alpha \beta}^{R}\left(\boldsymbol{\sigma}_{1} \cdot \boldsymbol{\sigma}_{2}\right)\right)}{\text { contact terms }}+\frac{f_{\pi N N}^{2} \mathcal{T}_{\alpha \beta}^{R}\left(\boldsymbol{\sigma}_{1} \cdot \boldsymbol{k}\right)\left(\boldsymbol{\sigma}_{2} \cdot \boldsymbol{k}\right)}{\text { one-pion exchange }} \\
& \text { Landau-Migdal parameters } \\
& \qquad \begin{array}{l}
\mathcal{F}_{\alpha \beta}^{R}=f_{\alpha \beta} \gamma\left(f_{\alpha \beta}\right) \\
\mathcal{Z}_{n n}^{R}=g_{n n} \gamma\left(g_{n n}\right)
\end{array} \\
& \mathcal{Z}_{n p}^{R}=g_{n p} \gamma\left(g_{n n}\right)
\end{aligned}
$$

\section{Re-summed $N N$ interaction}

Non-resonance processes: As follows from numerical estimates, the main contribution to NN interaction at $n>n_{0}$ is given by Modified One-Pion Exchange (MOPE) not by Free One-Pion Exchange (FOPE)

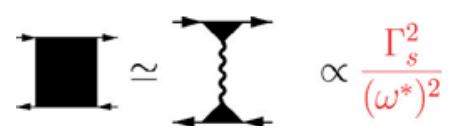

if the spin-isospin channel, $\sim\left(\sigma_{1} \cdot \boldsymbol{k}\right)\left(\sigma_{2} \cdot \boldsymbol{k}\right)$, is not forbidden or suppressed by symmetry reasons, small momentum transfer, etc.

\section{Two important ingradients of MOPE interaction:}

pion softening: $\quad$ pion gap $\left[\omega^{*}\left(n=n_{0}\right)\right]^{2} \simeq m_{\pi}^{2}$ instead of $m_{\pi}^{2}+p_{\mathrm{F}, N}^{2} \simeq 7 m_{\pi}^{2}$ in FOPE vertex suppression factor: $\quad \Gamma\left(n_{0}\right) \simeq 0.3-0.4$

Compensation of the repulsion $\Gamma^{2}$ and attraction $\omega^{*}$ for $n=n_{0}$ but sharp density dependence of the interaction! 


\section{Re-summed weak interaction}

The weak coupling vertex is renormalized in medium:

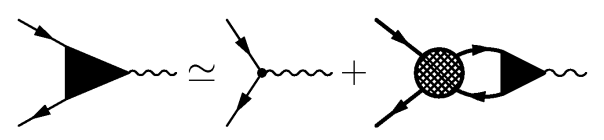

wavy line corresponds to weak current

For the $\beta$-decay:

$$
V_{\beta}=\frac{G}{\sqrt{2}}\left[\widetilde{\gamma}\left(f^{\prime}\right) l_{0}-g_{\Lambda} \widetilde{\gamma}\left(g^{\prime}\right) \boldsymbol{l} \boldsymbol{\sigma}\right]
$$

For processes on the neutral currents $\quad N_{1} N_{2} \rightarrow N_{1} N_{2} \nu \bar{\nu}$

with the correlation functions

$$
\begin{aligned}
V_{n n} & =-\frac{G}{2 \sqrt{2}}\left[\gamma\left(f_{n n}\right) l_{0}-g_{A} \gamma\left(g_{n n}\right) \boldsymbol{l} \boldsymbol{\sigma}\right] \\
V_{p p}^{N} & =\frac{G}{2 \sqrt{2}}\left[\kappa_{p p} l_{0}-g_{A} \gamma_{p p} \boldsymbol{l} \boldsymbol{\sigma}\right]
\end{aligned}
$$

$$
\kappa_{p p}=c_{V}-2 f_{n p} \gamma\left(f_{n n}\right) C_{0} L_{n n}, \gamma_{p p}=\left(1-4 g C_{0} L_{n n}\right) \gamma\left(g_{n n}\right),
$$

[Voskresensky, Senatorov, Sov. J. Nucl. Phys. 45 (1987)]

\section{Re-summed weak interaction}

Renormalization of the proton vertex (vector part of $V_{p p}^{N}+V_{p p}^{\gamma}$ ) is governed by processes

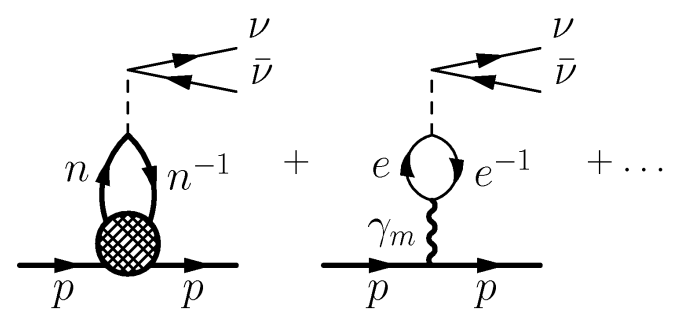

which are absent in vacuum

The squared matrix element related to the proton vector current term is $\propto c_{V}^{2}$ in vacuum, but in medium it is $\propto \kappa_{p p}^{2}$ ( first diagram)

$\Rightarrow$ enhancement up to $\sim 10-100$ times for $1.5-3 n_{0}$

[Voskresensky, Senatorov, Sov. J. Nucl. Phys (1987)]

Another enhancement factor (up to $\sim 10^{2}$ ) comes from the virtual photon $\left(\gamma_{m}\right)$

dressded in medium (second diagram)

[Voskresensky, Kolomeitsev, Kampfer Sov. JETP, 87 (1998); Leinson, Phys. Lett. B (2000)] 


\section{Re-summed weak interaction}

Another example of the correlation effect:

In vacuum the branching ratio of the kaon dacays is

$$
\frac{\Gamma\left(K^{-} \rightarrow e^{-}+\nu_{e}\right)}{\Gamma\left(K^{-} \rightarrow \mu^{-}+\nu_{\mu}\right)} \approx 2.5 \times 10^{-5}
$$

In medium this ratio can be of the order of unity due to $\Lambda p^{-1}$ decays of virtual $K^{-}$. In dependence of the reaction channel, in-medium effects may lead to strong enhancement or suppression of reaction rates

Neglect of these effects may lead to misleading results

\section{Medium effects in two-nucleon processes, MMU}

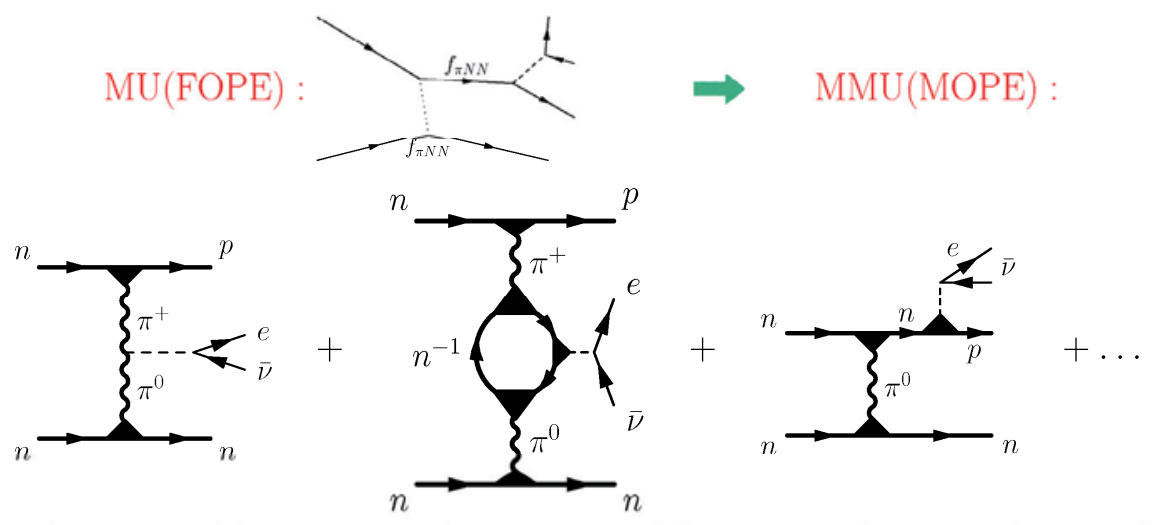

First diagram yields main contribution, second diagram - a less contribution, third diagram ( generalizes the MU(FOPE)) yields much less term for $n \gtrsim n_{0}$. pion softening $\Rightarrow$ enhancement of the rate towards the $n_{c}^{\mathrm{PU}}$.

$$
\frac{\epsilon_{\nu}[\mathrm{MMU}]}{\varepsilon_{\nu}[\mathrm{MU}]} \sim 10^{3}\left(n / n_{0}\right)^{10 / 3} \frac{\Gamma^{6}(n)}{\left[\omega^{*}(n) / m_{\pi}\right]^{8}} . \quad \begin{gathered}
\text { Very strong density } \\
\text { dependence }
\end{gathered}
$$




\section{Medium effects in two-nucleon processes, MNB}
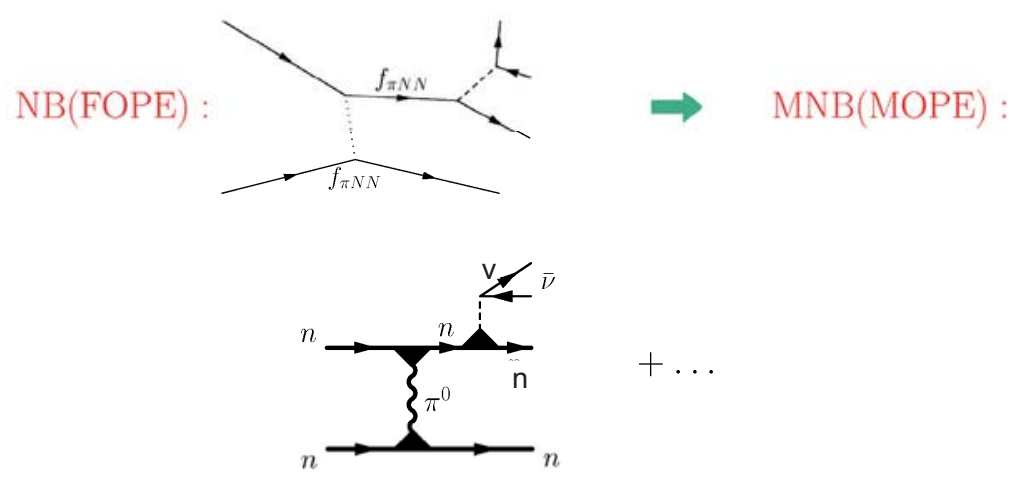

pion softening $\Rightarrow$ enhancement of the rate towards the $n_{c}^{\mathrm{PU}}$.

$$
\frac{\varepsilon_{\nu}[\mathrm{MNB}]}{\varepsilon_{\nu}[\mathrm{NB}]} \sim 10^{3}\left(n / n_{0}\right)^{4 / 3} \frac{\mathrm{I}^{6}(n)}{\left[\omega^{*}(n) / m_{\pi}\right]^{3}} .
$$

A different enhancement factor for the MNB processes compared to MMU.

\section{Proper DU processes}
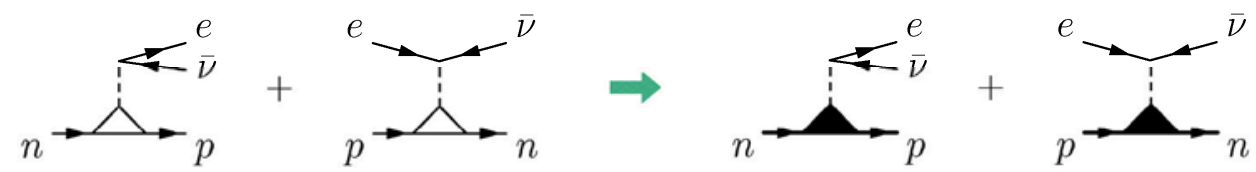

They are forbidden up to the density $n_{c}^{\mathrm{DU}}$ when triangle inequality $p_{\mathrm{Fn}}<p_{\mathrm{Fp}}+p_{\mathrm{Fe}}$ begins to fulfill. For traditional EoS like $V 18+\delta v+U I X^{*}$ DU processes are permitted only for $n>5 n_{0}$.

Due to full vertices $\quad \rightarrow \quad$ a factor $\Gamma_{\mathrm{w}-\mathrm{S}}^{2}$ in emissivity.

(rather minor modification, since $\omega \simeq p_{\mathrm{F}, e} \gg q \sim T$ ). 


\section{DU-like processes (on condensates)}

For $n>n_{c}^{\mathrm{PU}}\left(M>M_{c}^{\mathrm{PU}}\right) \mathrm{PU}$ processes:
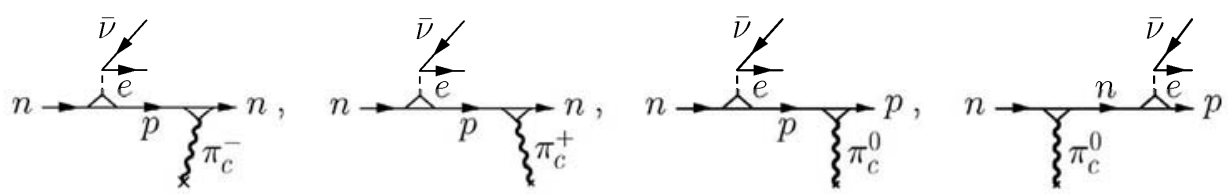

with free vertices: $\epsilon_{\nu} \sim 10^{26} T_{9}^{6}\left(n / n_{0}\right)^{1 / 3} \frac{\mathrm{erg}}{\mathrm{cm}^{3} \mathrm{soc}}$
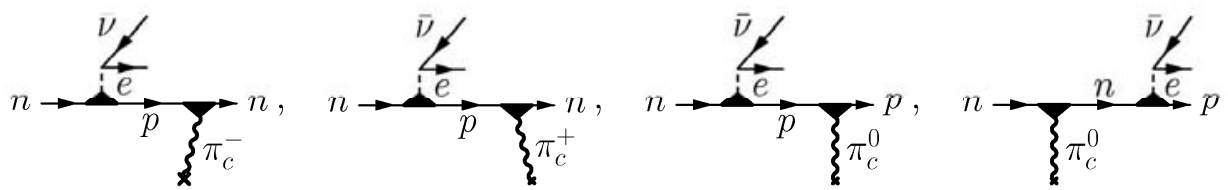

with full vertices: $\epsilon_{\nu} \sim 10^{26} \Gamma_{s}^{2} \Gamma_{\mathrm{w}-\mathrm{s}}^{2} T_{9}^{6}\left(n / n_{0}\right)^{1 / 3} \frac{\mathrm{erg}}{\mathrm{cm}^{3} \mathrm{sec}}$ $\stackrel{\mathrm{sec}}{\Rightarrow} \Gamma_{s}^{2} \Gamma_{\mathrm{w}-\mathrm{s}}^{2} \sim 10^{-1}-10^{-2}$

\section{DU-like processes (on condensates)}

- at $n>n_{c}^{\mathrm{KU}} \gtrsim(3-4) n_{0}\left(M>M_{c}^{\mathrm{KU}}\right)$

kaon Urca (KU) processes on charged and neutral kaon condensation may occur.

Kaon condensates can be created in reactions

$$
e+X \rightarrow K^{-}+X^{\prime}, n+X \rightarrow p+K^{-}+X^{\prime}, n+X \rightarrow n+\bar{K}^{0}+X^{\prime} \text {, etc. }
$$

$K^{-}$condensate can arise in $s$-wave

[Kaplan, Nelson, PLB175 (1986);

Brown, Kubodera, Page, Pizzochero, PRD37 (1988)]

or in p-wave

[Kolomeitsev, Voskresensky, Kampfer, NPA588 (1995);

Kolomeitsev, Voskresensky, PRC68 (2003)]

extra $\sin ^{2} \theta_{C} \simeq(0.23)^{2}$ suppression factor in emissivity,

and a different correlation factor (poorly known constants)

\section{Charged $\rho$ condensate}

[Voskresensky, Phys.Lett. B392 (1997),

Kolomeitsev, Voskresensky, NPA759 (2005)

if $\rho$-meson is treated as non-Abelian boson

and its effective mass decreases in dense matter (below half of $m_{\rho}$ )

Neutrino production processes are similar to those for $\pi^{-}$condensate.

Emissivity is estimated as: $\epsilon_{\nu}^{\mathrm{RU}} \sim 10^{26} g_{\rho N}^{* 2}\left(\frac{m_{N}^{*}}{m_{N}}\right)^{2} \frac{\mu_{e}}{m_{N}} \frac{n_{c h}^{\rho}}{m_{\rho}^{*}} \Gamma_{s}^{2} \Gamma_{\mathrm{w}-\mathrm{s}}^{2} T_{9}^{6}, \frac{\mathrm{erg}}{\mathrm{cm}^{3} \mathrm{sec}}$ 


\section{Other resonance processes}

[Voskresensky, Khodel, Zverev, Clark, AJ, 533 (2000)]

- Bubble rearrangement of the Fermi sphere in the vicinity of the $\pi^{0}$ condensation critical point. Occurs if necessary condition of stability of the normal state

$$
\delta E_{0}=\int[\epsilon(p, n(p))-\mu] \delta n(p) \frac{d^{3} p}{(2 \pi)^{3}}>0
$$

is violated (when a depression $\epsilon(p, n(p))-\mu<0$ forms for $p>p_{\mathrm{F}}$ or elevation $\epsilon(p, n(p))-\mu>0$, for $\left.p<p_{\mathrm{F}}\right)$. Numerical study shows that due to strong attraction in pion channel of $N N$ interact. for $n_{c 1}<n<n_{c}^{\pi^{0}}$ there arises a bubble (absence of particles) in neutron distrib. for $p_{i}<p<p_{f}<p_{\mathrm{F}}$ and triangle inequality is fulfilled.

$\Rightarrow$ DU-like process $\epsilon_{\nu} \sim 10^{27} T_{9}^{6} . \quad \Rightarrow T^{6}$ dependence.

- Competing possibility: fermion condensation.

Also new neutron distribution $0<n(p)<1$ for $p<p_{\mathrm{F}}$ is found in the range $n_{\mathrm{c} 1}<n<n_{c}^{\pi^{0}}$ from condition $\delta E_{0} / \delta n(p)=\mu$ for $p_{i}<p<p_{f}<p_{\mathrm{F}}$. It produces $d m_{n} / d T \propto 1 / T$ for $T \rightarrow 0$.

$\Rightarrow$ DU-like process $\epsilon_{\nu} \sim 10^{27} T_{9}^{5}$ with $T^{5}$ dependence, more efficient than DU.

\section{Other resonance processes}

There are many other in-medium reaction channels, e.g., with zero sound excitations.

- The most essential contribution comes from the neutral current processes

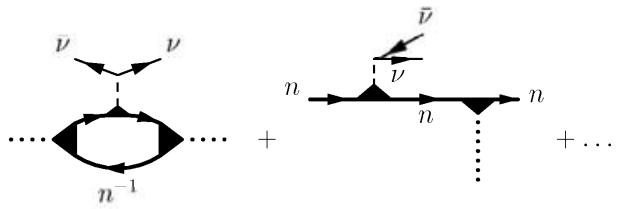

The dotted line is zero sound quantum of appropriate symmetry. These are resonance processes (second, of DU-type) similar to processes going on condensates. Difference: rates of reactions with zero sounds are $\propto$ to thermal occupations of the corresponding spectrum branches. Contribution of the resonance reactions with zero sounds is suppressed due to a small phase space volume $(q \sim T)$, cf. phonon processes. 


\section{Relations between one-, two-, etc. nucleon processes}

Within the QPA for fermions, the reaction rate with participation of one fermion and the boson is given by [D.V., Senatorov JETP Lett. 40 (1984); JETP 63 (1986)]

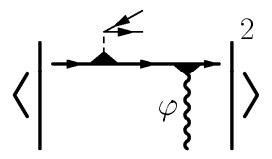

For equilibrium system $(T \neq 0)$ there is exact relation

$$
\left.\left(<\hat{\varphi}_{2}^{\dagger} \hat{\varphi}_{1}\right\rangle\right)(p)=i D^{+}+\left|\varphi_{c}\right|^{2}=\frac{A_{B}}{\operatorname{cxp}\left(\frac{\omega}{T}\right)-1}+\left|\varphi_{c}\right|^{2}, \quad A_{B}=-2 \operatorname{Im} D^{R},
$$

$A_{B}$ contains the $\delta$-function at spectrum branches related to resonance proc. , like zero sound, and also a contrib. $\propto \operatorname{Im} \Pi^{R}$ of the loop diagram. Within the QPA taking Im part is related to a cut of the diagram. $\Rightarrow$ The same term can be calculated via

i.e. through two-nucleon process.

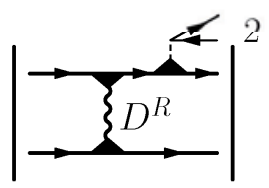

\section{Optical theorem again}

Perturbative diagrams are irrelevant for calculation of in-medium processes. In general case one should deal with closed diagrams in terms of dressed Green's functions [D.V., Senatorov Sov. Nucl. Phys. 45 (1987); Knoll, D.V. Ann. Phys. 249 (1996)].

$$
\left.\frac{d \mathcal{W}_{\bar{\nu} l}^{\text {tot }}}{d t}=\frac{\left(1-n_{l}\right) d^{3} q_{\bar{\nu}} d q_{l}^{3}}{(2 \pi)^{6} 4 \omega_{\bar{\nu}} \omega_{l}}\left(N_{\bar{\nu}}^{l} N=1\right)_{l}^{\bar{\nu}}+N_{l}^{l}+\ldots\right) .
$$

Series in number of full $\{-,+\}$ Green func. (in terms of non-eq. diag. tech.). Only if QPA for fermions is relevant (i.e. for $T \ll \epsilon_{\mathrm{FN}}$ ) one can do a cut of fermion lines, thus considering diagrams with open fermion legs:

- not the case for pions,

- not the case for the description of initial stage of NS evolution (not small $T$ )

- For $T<T_{c}$ closed diagrams with two types of fermion Green's functions: normal and anomalous ones. 


\section{Superfluidity. DU-like processes. MNPBF processes}

Permitted only for $T<T_{c} \underset{N}{\Lambda_{N}^{\nu}} \rightarrow$

Renormalization of the proton vertex (vector part of $V_{p p}^{N}+V_{p p}^{\gamma}$ ) is governed by processes

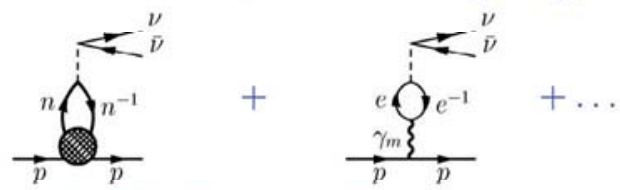

forbidden in vacuum. $\Rightarrow 10^{2}$ enhancement! [Voskresnsky, Senatorov (1987)]

[Schaab, Voskresensky, Sedrakian, Weber, Weigel, AA (1997);

incorporated in cooling code

Blaschke, Grigorian, Voskresensky, AA (2001, 2004);

Grigorian, Voskresensky, AA (2005)]

Both for neutrons and protons:

$$
\epsilon_{\nu} \sim 10^{29}\left[\frac{\Delta_{n n}}{\mathrm{MeV}}\right]^{7}\left[\frac{T}{\Delta_{n n}}\right]^{1 / 2}\left(n / n_{0}\right)^{1 / 3} \xi_{\text {ii }}^{2}, \frac{\mathrm{erg}}{\mathrm{cm}^{3} \mathrm{sec}},
$$

$\Delta_{i i}$ is $N N$ gap, $i=n, p, \xi_{i i}=\exp \left[-\Delta_{i i} / T\right]$ is superfluid suppression factor.

\section{PBF in optical theorem formalism}

We may explicitly decompose the first term in the series as

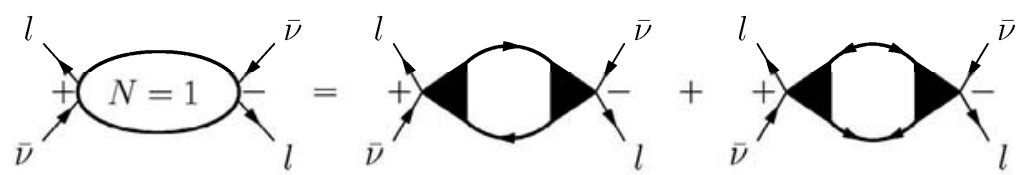

$\Rightarrow$ PFB processes are of the one-nucleon origin determined by $N=1$ diagrams.

Due to that a huge $10^{29}$ pre-factor in emissivity.

- QP Green functions and dressed vertices. Due to dressed vertices $\sim 10^{2}$ factor for MpPFB. 


\section{Superfluidity. Massive photon decay}

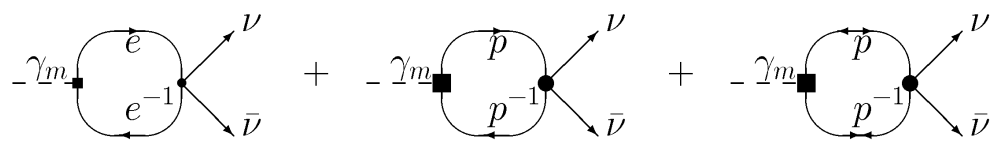

$$
\varepsilon_{\nu}^{\gamma} \approx 2.6 \cdot 10^{25} T_{9}^{\frac{3}{2}} e^{-\frac{m_{\gamma}}{T}}\left(\frac{m_{\gamma}}{\mathrm{MeV}}\right)^{\frac{7}{2}}\left(\frac{n}{n_{0}}\right)^{\frac{8}{3}}\left(1+\frac{3}{2} \frac{T}{m_{\gamma}}\right) \frac{\mathrm{erg}}{\mathrm{cm}^{3} \mathrm{sec}}
$$

Again, only "normal" correlations were incorporated in vertices

[Voskresensky, Kolomeitsev, Kaempfer, JETP 87 (1998)]

\section{Breaking and Formation of Cooper pairs (PBF)}

In superfluid $\left(T<T_{c}<0.1-1 \mathrm{MeV}\right)$ all two-nucleon processes are suppressed by factor $\exp (-2 \Delta T)$

new "quasi"-one-nucleon-like processes (one-nucleon phase space volume)

become permitted

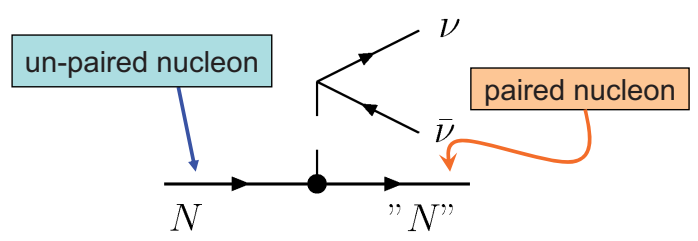




\title{
Neutrino emission reactions
}

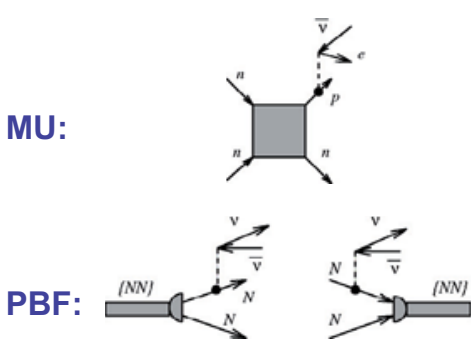

DU:

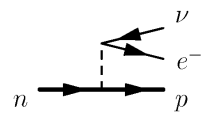

PU:

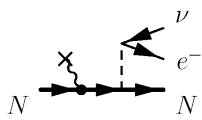

$\underline{\text { minimal }} \underline{T<T_{\text {opac }} \sim 10^{-1} \div 10^{0} \mathrm{MeV}}$

$$
10^{28} \times\left(\frac{T}{\mathrm{MeV}}\right)^{8}\left(\frac{n_{e}}{n_{0}}\right)^{\frac{1}{3}} \quad \frac{\mathrm{erg}}{\mathrm{cm}^{3} \mathrm{~s}} \times e^{-2 \Delta / T}
$$$$
10^{28} \times\left(\frac{\Delta}{\mathrm{MeV}}\right)^{7}\left(\frac{T}{\Delta}\right)^{\frac{1}{2}} e^{-2 \Delta / T} \frac{\mathrm{erg}}{\mathrm{cm}^{3} \mathrm{~s}}
$$$$
\text { dominates for } T \ll \Delta \quad \text { (at least) }
$$

\section{exotic}

$10^{27} \times T_{9}^{6}\left(\frac{n_{e}}{n_{0}}\right)^{\frac{1}{3}} \frac{\mathrm{erg}}{\mathrm{cm}^{3} \mathrm{~s}} \times e^{-\Delta / T}$

allowed if $n>n_{c}^{\mathrm{DU}}$

$10^{27} \times T_{9}^{6} \frac{\left|\varphi_{c}\right|^{2}}{m_{\pi}^{2}} \frac{\mathrm{erg}}{\mathrm{cm}^{3} \mathrm{~s}} \times e^{-\Delta / T}$

allowed if $n>n_{c}^{\mathrm{PU}}$

\section{NEUTRINO PAIR EMISSION FROM FINITE-TEMPERATURE NEUTRON SUPERFLUID AND THE COOLING OF YOUNG NEUTRON STARS*}

\author{
ELLIOTT FLOWERS \\ Department of Physics, New York University \\ MALVIN RUdERMAN \\ Department of Physics, Columbia University \\ AND \\ Peter Sutherland \\ Department of Physics, University of Pennsylvania \\ Received 1975 August 29
}

\begin{abstract}
its critical temperature $T_{\mathrm{c}}$ for the transition to the superfluid state. At any nonzero temperature $T<T_{\mathrm{c}}$ the neutron fluid has two components: a superfluid condensate and quasiparticle excitations (broken "Cooper pairs"). We calculate the neutrino pair emissivity due to the recombination of broken pairs that then join the condensate. ${ }^{1}$ Again, this process depends on the existence of a weak neutral current that permits transitions between states of differing numbers of broken-pair excitations. We find the resulting emissivity to be (in units with $\hbar=c=1$ ):

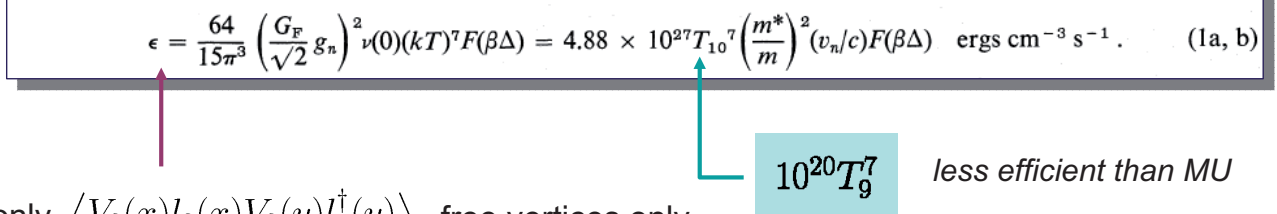

only $\left\langle V_{0}(x) l_{0}(x) V_{0}(y) l_{0}^{\dagger}(y)\right\rangle$ free vertices only
\end{abstract}




\title{
Descripiivu vi a ruciear interaciion in the Keidysh diagram technique and the problem of neutrino luminosity of neutron stars
}

D. N. Voskresenskii and A. V. Senatorov

Moscow Engineering-Physics Institute

(Submitted 20 February 1986)

Yad. Fiz. 45, 657-669 (March 1987)
IV. GENERALIZATION OF THE OPTICAL-THEOREM FORMALISM IN THE KELDYSH DIAGRAM TECHNIQUE TO SYSTEMS WITH PAIRING. CALCULATION OF THE LUMINOSITY OF THE ONE-NUCLEON PROCESSES $n_{\mathrm{pa}} \rightarrow n v \bar{v}$ and $p_{\rho \mathrm{p}} \rightarrow \rho v \bar{v}$<smiles>CC(C)C1CC2CCC(C2)C1C(C)C</smiles>

a<smiles>CC(C)C1C2CC3C(C2)C1C3C(C)C</smiles>

$\mathrm{b}$

closed diagram technique

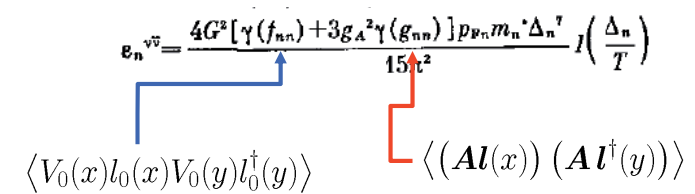
no contribution for s pairing

$$
\begin{gathered}
\epsilon_{\nu} \sim 10^{28}\left[\frac{\Delta_{n n}}{\mathrm{MeV}}\right]^{7}\left[\frac{T}{\Delta_{n n}}\right]^{1 / 2}\left(n / n_{0}\right)^{1 / 3} \xi_{n n}^{2}, \frac{\mathrm{erg}}{\mathrm{cm}^{3} \mathrm{sec}} \\
\Delta_{\mathrm{nn}} \text { is neutron gap and } \xi_{\mathrm{nn}}=\exp \left(-\Delta_{\mathrm{nn}} / \mathrm{T}\right)
\end{gathered}
$$

One-nucleon phase-space, dominant over $\mathrm{MU}$ for $\mathrm{T}<<2 \Delta$

\section{9}

Astron. Astrophys. 343, 650-660 (1999)

\section{ASTRONOMY \\ AND \\ ASTROPHYSICS}

\section{Neutrino emission due to Cooper pairing of nucleons in cooling neutron stars}

\author{
D.G. Yakovlev, A.D. Kaminker, and K.P. Levenfish \\ Ioffe Physical Technical Institute, Politekhnicheskaya 26, 194021 St.-Petersburg, Russia
}

Received 18 September 1998 / Accepted 29 October 1998

singlet and triplet pairing

$$
\begin{array}{ll}
\left\langle V_{0}(x) l_{0}(x) V_{0}(y) l_{0}^{\dagger}(y)\right\rangle & \begin{array}{l}
\text { calculated using nucleon wave functions } \\
\text { and Bogolubov transformations }
\end{array} \\
& \text { free vertices }
\end{array}
$$

relativistic corrections for axial current contribution 


\section{Vector current conservation}

$\underline{\text { normal matter }}$

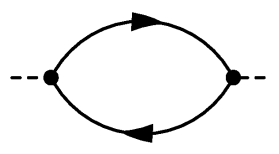

free vertices and Green's functions

The WT identity is fulfilled and the current is conserved

$\underline{\text { superfluid matter }}$

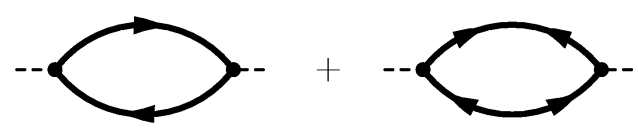

free vertices and non-interacting quasi-particles

with a gapped spectrum $E_{p}^{2}=\epsilon_{p}^{2}+\Delta^{2}$

Gap appears due to a non-trivial self-energy

Vertex must be modified accordingly. Otherwise the current is not conserved
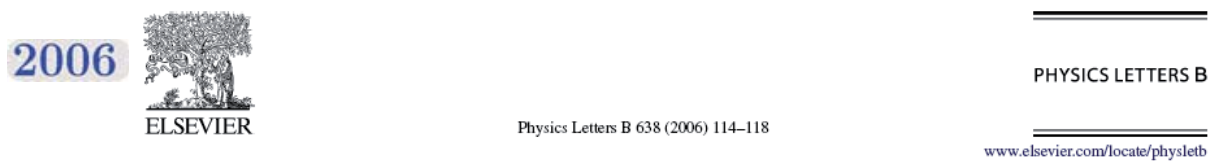

Vector current conservation and neutrino emission from singlet-paired baryons in neutron stars

L.B. Leinson ${ }^{\mathrm{a}, \mathrm{b}}$, A. Pérez ${ }^{\mathrm{b}, *}$

a Institute of Terrestrial Magnetism, Ionosphere and Radio Wave Propagation, RAS, 142190 Troitsk, Moscow Region, Russia

${ }^{\mathrm{b}}$ Departamento de Fisica Teórica and IFIC, Universidad de Valencia-CSIC, Dr. Moliner 50, 46100-Burjassot, Valencia, Spain

vector current conservation $\quad q_{\mu} V^{\mu}=0 \quad V_{0}=\frac{1}{\omega} \boldsymbol{q} \boldsymbol{V}$

$$
\left\langle V_{0} V_{0}\right\rangle=\frac{\boldsymbol{q}^{2}}{\omega^{2}}\langle\boldsymbol{V} \boldsymbol{V}\rangle \sim O\left(\boldsymbol{q}^{2} v_{\mathrm{F}}^{2} / \omega^{2}\right) \quad \text { at least ! }
$$

Conclusion:

Thus the neutrino energy losses due to singlet-state pairing of baryons can, in practice, be neglected in simulations of neutron star cooling. This makes unimportant the neutrino radiation from pairing of protons or hyperons.

calculations are done in the limit $\omega<<\Delta$, whereas for PFB processes $\omega \sim 2 \Delta$ 


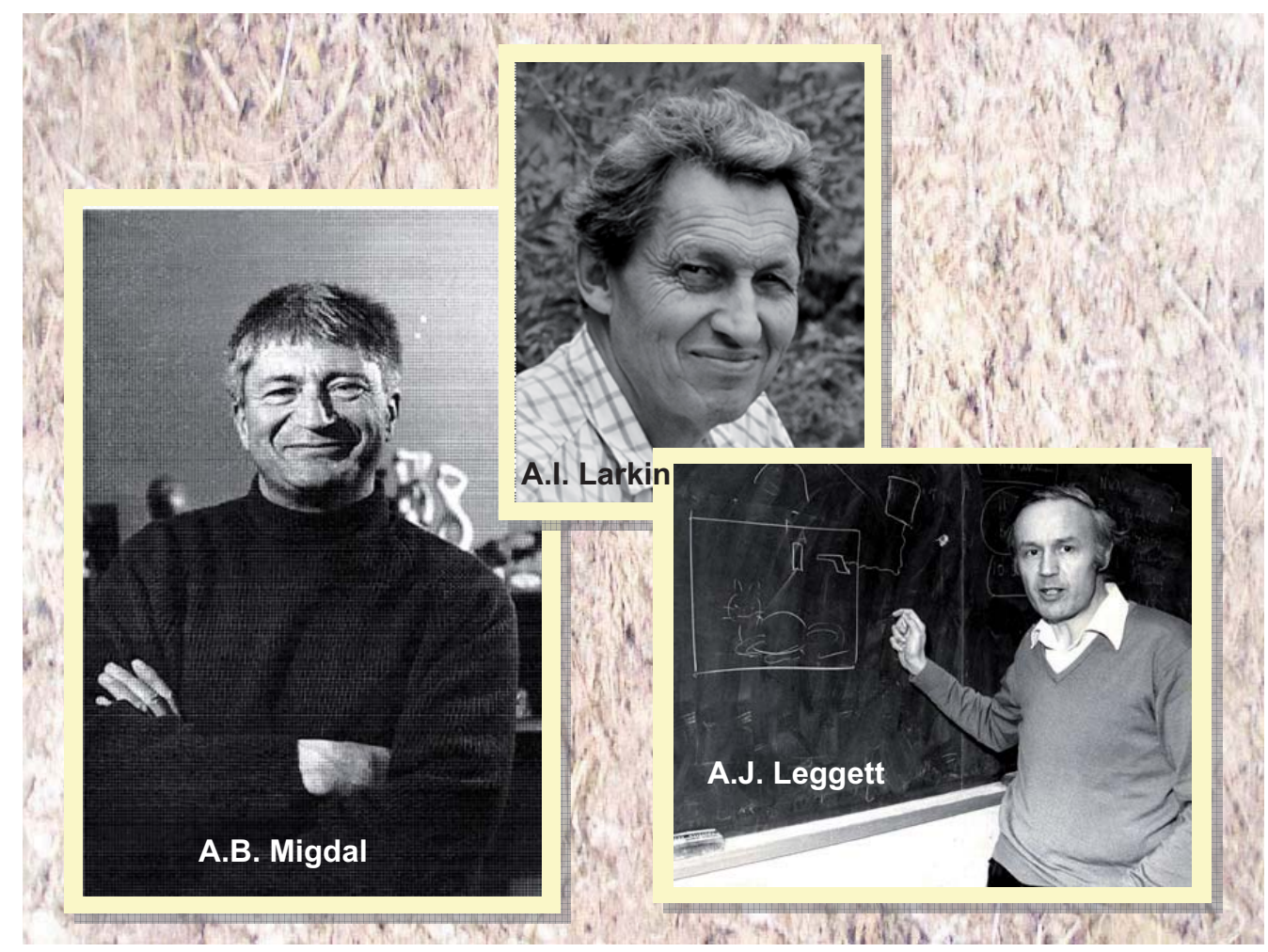

\section{Neutrino emissivity in superfluid Fermi liquid}

Consider pure neutron matter at $T<<2 \Delta$

$$
\begin{aligned}
& \qquad \varepsilon_{\nu \bar{\nu}}=\frac{G^{2}}{8} \int \frac{d^{3} q_{1}}{(2 \pi)^{3} 2 \omega_{1}} \frac{d^{3} q_{2}}{(2 \pi)^{3} 2 \omega_{2}} \omega \frac{n_{\mathrm{bos}}(\omega)}{\uparrow} 2 \operatorname{Im} \sum_{\text {lept. spin }} \chi(q) \\
& \text { produces leading exponential term } \propto e^{-2 \Delta / T}
\end{aligned}
$$

$$
\text { closed diagrams calculated with Green's functions for } T=0
$$




\section{Superfluid system}

- Normal Green's functions

particle $\quad i \hat{G}=$

hole $\quad i \hat{G}^{h}(p)=$

$\hat{G}^{h}(p)=\sigma_{2}\left[\hat{G}^{h}(p)\right]^{\mathrm{T}} \sigma_{2}=i \hat{G}(-p)$

Number of excitations is not conserved !

amplitude of 2 particle annihilation

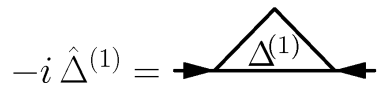

amplitude of 2 particle creation

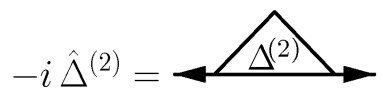

- Anomalous Green's functions

describe transformation particles into holes and vice versa particle $\rightarrow$ hole $i \hat{F}^{(1)}=$ hole $\rightarrow$ particle $i \hat{F}^{(2)}=$

\section{Superfluid system}

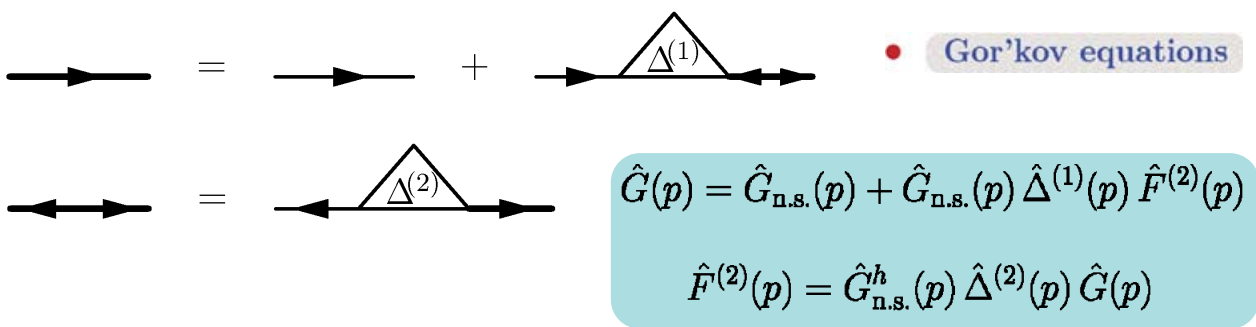

- Gap equation

$$
\left[\hat{\Delta}^{(1)}\right]_{b}^{a}=\int \frac{d^{4} p^{\prime}}{(2 \pi)^{4} i}\left[\widehat{V}\left(p, p^{\prime}\right)\right]_{b d}^{a c}\left[\hat{G}\left(p^{\prime}\right) \hat{\Delta}^{(1)}\left(p^{\prime}\right) \hat{G}_{\mathrm{n.s.}}^{h}\left(p^{\prime}\right)\right]_{c}^{d}
$$

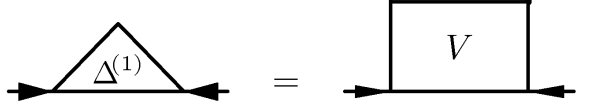

$[\widehat{V}]_{b d}^{a c}=V_{0}\left(i \sigma_{2}\right)_{b}^{a}\left(i \sigma_{2}\right)_{d}^{c}+V_{1}\left(i \sigma_{2} \boldsymbol{\sigma}\right)_{b}^{a}\left(\boldsymbol{\sigma} i \sigma_{2}\right)_{d}^{c}$ 


\section{Superfluid system}

- Coupling to an external field
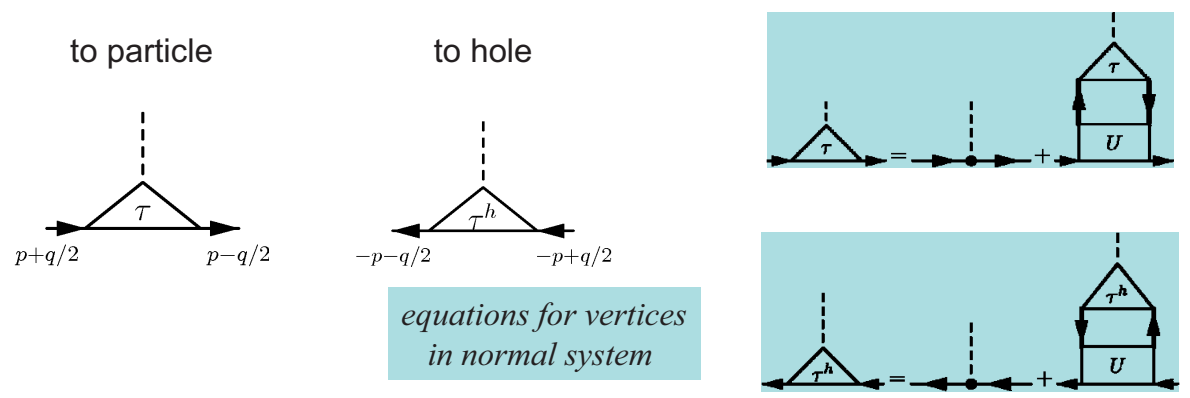

In superfluid systems new type of couplings:

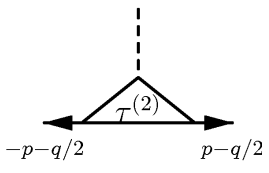

ext. field create 2 particles

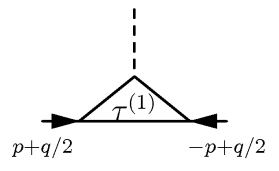

ext. field create 2 holes

\section{Equations for vertices}

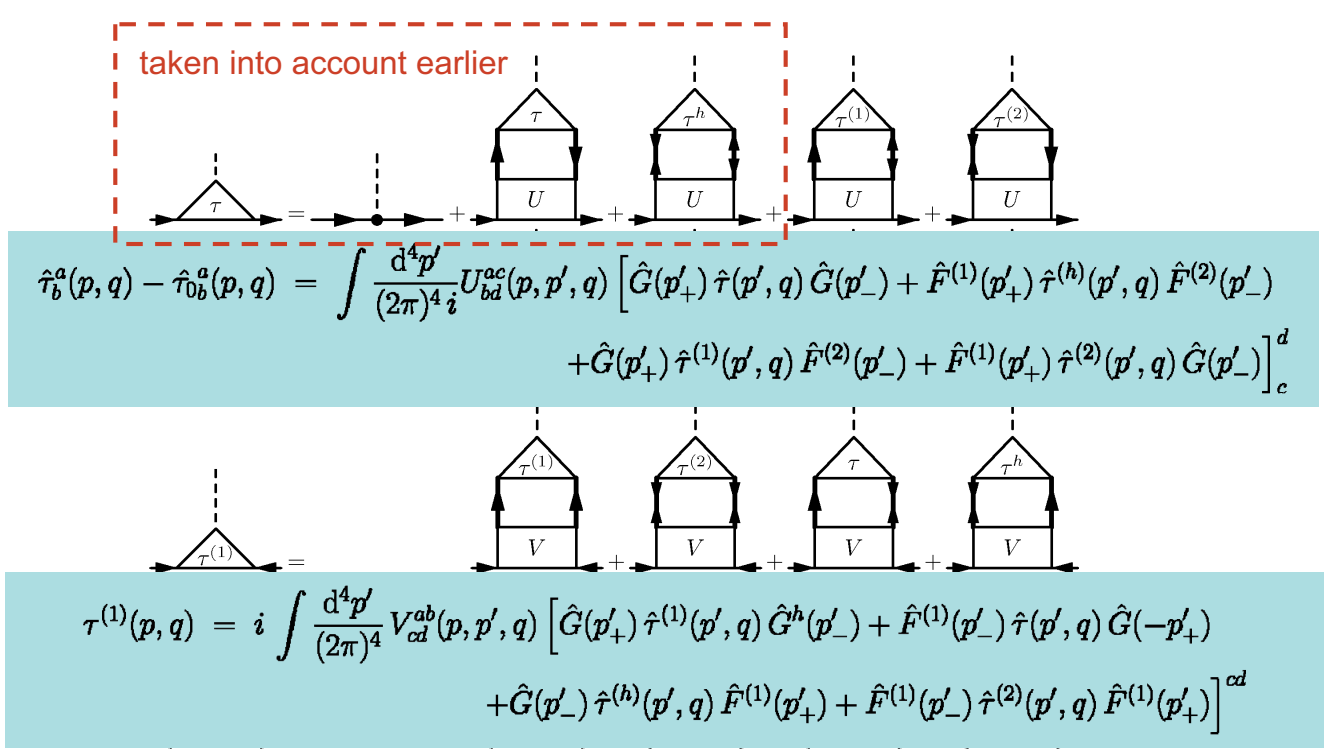

Cannot be written in matrix form in Nambu Gor'kov space since $U=/=V$ 


\section{Fermi liquid approximation}

S wave pairing $\quad \hat{\Delta}^{(1)}=\hat{\Delta}^{(2)}=\Delta i \sigma_{2} \quad \hat{F}^{(1)}=\hat{F}^{(2)}=F i \sigma_{2}$

pole parts of the Green's functions

$$
\begin{gathered}
G(p)=\frac{a\left(\epsilon+\epsilon_{p}\right)}{\epsilon^{2}-E_{p}^{2}+i 0 \operatorname{sgn} \epsilon} \quad F(p)=\frac{-a \Delta}{\epsilon^{2}-E_{p}^{2}+i 0 \operatorname{sgn} \epsilon} \\
\epsilon_{p} \approx \frac{p^{2}-p_{\mathrm{F}}^{2}}{2 m_{N}^{*}} \approx v_{\mathrm{F}}\left(p-p_{\mathrm{F}}\right) \longrightarrow \text { "gapped" spectrum } \quad E_{p}^{2}=\epsilon_{p}^{2}+\Delta^{2}
\end{gathered}
$$

\section{Landau-Migdal parameters}

particle-hole interaction:

$$
\left[\widehat{\Gamma}^{\omega}\right]_{b d}^{a c}=\Gamma_{0}^{\omega}\left(\boldsymbol{n}, \boldsymbol{n}^{\prime}\right) \delta_{b}^{a} \delta_{d}^{c}+\Gamma_{1}^{\omega}\left(\boldsymbol{n}, \boldsymbol{n}^{\prime}\right)(\boldsymbol{\sigma})_{b}^{a}(\boldsymbol{\sigma})_{d}^{c} .
$$
repulsive (stability of Fermi liquid)

particle-particle interaction:

$$
\begin{array}{r}
{\left[\widehat{\Gamma}^{\xi}\right]_{b d}^{a c}=\Gamma_{0}^{\xi}\left(\boldsymbol{n}, \boldsymbol{n}^{\prime}\right)\left(i \sigma_{2}\right)_{b}^{a}\left(i \sigma_{2}\right)_{d}^{c}+\Gamma_{1}^{\xi}\left(\boldsymbol{n}, \boldsymbol{n}^{\prime}\right)\left(i \sigma_{2} \boldsymbol{\sigma}\right)_{b}^{a}\left(\boldsymbol{\sigma} i \sigma_{2}\right)_{d}^{c}} \\
\underline{\text { attractive }}(\text { to allow pairing of fermions) }
\end{array}
$$

\section{Fermi liquid approximation}

integration over the internal lines is reduced to the Ferm surface

$$
\int \frac{2 \mathrm{~d}^{4} p}{(2 \pi)^{4} i} \simeq \int \frac{\mathrm{d} \Omega_{\boldsymbol{p}}}{4 \pi} \times \int \mathrm{d} \Phi_{p} \quad \int \mathrm{d} \Phi_{p}=\rho \int_{-\infty}^{+\infty} \frac{\mathrm{d} \epsilon}{2 \pi i} \int_{-\infty}^{+\infty} \mathrm{d} \epsilon_{p}
$$

$\rho=\frac{m^{*} p_{\mathrm{F}}}{\pi^{2}} \quad$ density states at Fermi surface

- Gap equation for $T \ll 2 \Delta$

$$
\Delta(\boldsymbol{n})=-A_{0}\left\langle\underline{\Gamma_{0}^{\xi}\left(\boldsymbol{n}, \boldsymbol{n}^{\prime}\right)} \Delta\left(\boldsymbol{n}^{\prime}\right)\right\rangle_{\boldsymbol{n}^{\prime}} \quad\langle\ldots\rangle_{\boldsymbol{n}}=\int \frac{\mathrm{d} \Omega_{\boldsymbol{n}}}{4 \pi}(\ldots)
$$

$A_{0}=\int \mathrm{d} \Phi_{p} G_{\mathrm{n} . \mathrm{s.}}(p) G^{h}(p) \theta\left(\xi-\epsilon_{p}\right) \approx a^{2} \rho \ln (2 \xi / \Delta)$

$\Gamma^{\xi}$ in an effective parameterization of a pairing gap 


\section{Fermi liquid approximation}

- Coupling to an external field

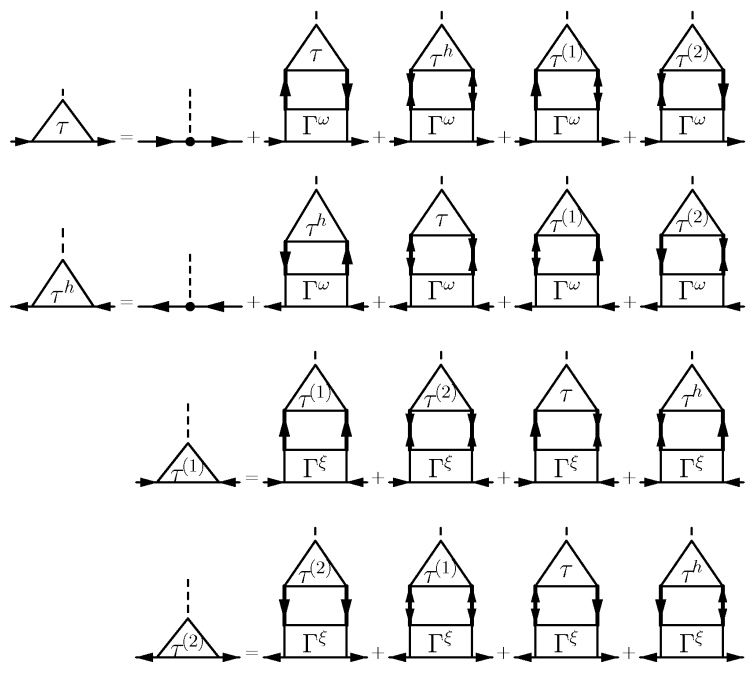

\section{Fermi liquid approximation}

- Current-current correlator

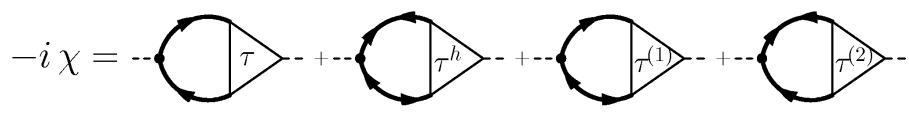

$$
\begin{aligned}
& \chi_{a}=\operatorname{Tr} \int \frac{d^{4} p}{(2 \pi)^{4} i} \hat{\tau}_{a}^{\omega}\left\{\hat{G}_{+} \hat{\tau}_{a}^{\dagger} \hat{G}_{-}+\hat{F}_{+}^{(1)} \hat{\tau}_{a}^{h \dagger} \hat{F}_{-}^{(2)}+\hat{G}_{+} \hat{\tau}_{a}^{(1) \dagger} \hat{F}_{-}^{(2)}+\hat{F}_{+}^{(1)} \hat{\tau}_{a}^{(2) \dagger} \hat{G}_{-}\right\} \\
& a=V, A \\
& G_{ \pm}=G(p \pm q / 2)
\end{aligned}
$$

$$
\begin{aligned}
& \chi_{V}(q)=g_{V}^{2}\left\langle\left(l_{0}-\boldsymbol{v} \boldsymbol{l}\right)\left(l_{0}^{\dagger} \chi_{V, 0}(\boldsymbol{n}, q)-\chi_{V, 1}(\boldsymbol{n}, q) \boldsymbol{l}^{\dagger}\right)\right\rangle_{\boldsymbol{n}} \\
& \chi_{A}(q)=g_{A}^{2} e_{A}^{2}\left\langle\left(l_{0} \boldsymbol{v}-\boldsymbol{l}\right)\left(l_{0}^{\dagger} \chi_{A, 1}(\boldsymbol{n}, q)-\chi_{A, 0}(\boldsymbol{n}, q) \boldsymbol{l}^{\dagger}\right)\right\rangle_{\boldsymbol{n}}
\end{aligned}
$$




\section{Solution for correlators}

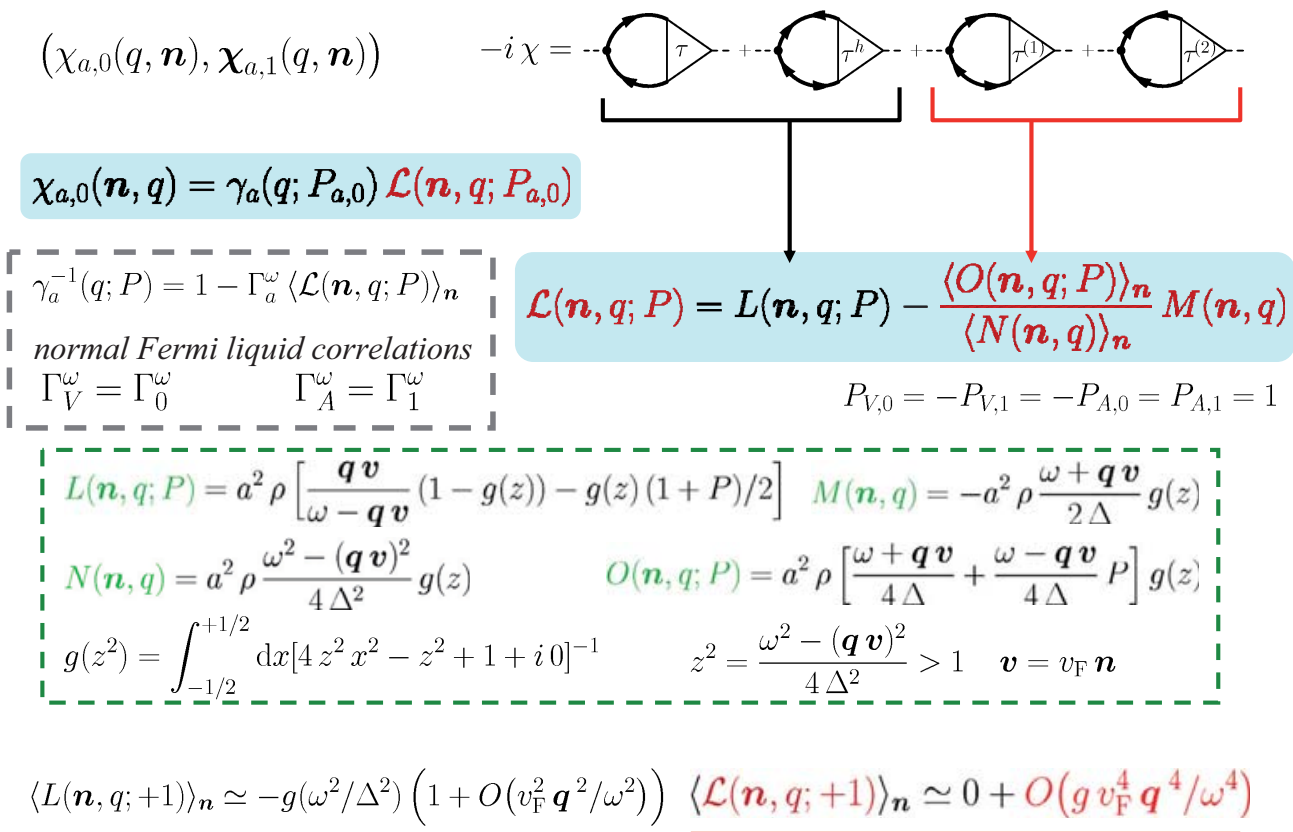

$\boldsymbol{\chi}_{a, 1}(\boldsymbol{n}, q)=\gamma_{a}\left(q ; P_{a, 1}\right) \boldsymbol{v} \mathcal{L}\left(\boldsymbol{n}, q ; P_{a, 1}\right)+\delta \boldsymbol{\chi}_{a, 1}(\boldsymbol{n}, q)$

$$
\begin{aligned}
& \delta \boldsymbol{\chi}_{a, 1}(\boldsymbol{n}, q)=\frac{M(\boldsymbol{n}, q)}{\left\langle N\left(\boldsymbol{n}^{\prime}, q\right)\right\rangle_{\boldsymbol{n}^{\prime}}}\left\langle O\left(\boldsymbol{n}^{\prime}, q ; P_{a, 1}\right)\left(\boldsymbol{v}-\boldsymbol{v}^{\prime}\right)\right\rangle_{n^{\prime}} \\
& \quad+\mathcal{L}\left(\boldsymbol{n}, q ; P_{a, 1}\right) \gamma_{a}\left(q ; P_{a, 1}\right) \Gamma_{a}^{\omega}\left\langle\widetilde{\mathcal{L}}\left(\boldsymbol{n}^{\prime}, q ; P_{a, 1}\right)\left(\boldsymbol{v}^{\prime}-\boldsymbol{v}\right)\right\rangle_{\boldsymbol{n}^{\prime}} \\
& \widetilde{\mathcal{L}}(\boldsymbol{n}, q ; P)=L(\boldsymbol{n}, q ; P)-\frac{\langle M(\boldsymbol{n}, q)\rangle_{\boldsymbol{n}}}{\langle N(\boldsymbol{n}, q)\rangle_{n}} O(\boldsymbol{n}, q ; P)
\end{aligned}
$$

$P_{V, 0}=-P_{V, 1}=-P_{A, 0}=P_{A, 1}=1$ 


\section{Vector current conservation}

4-vector $\left(\chi_{V, 0}(q, \boldsymbol{n}), \chi_{V, 1}(q, \boldsymbol{n})\right)$

$$
\operatorname{Im}\left\langle\tau_{V}^{\omega} \chi_{V}^{\nu}\right\rangle_{\boldsymbol{n}} q_{\nu}=O\left(f^{\omega} g \boldsymbol{q}^{6} v_{\mathrm{F}}^{6} / \omega^{6}\right)
$$

$$
\begin{array}{r}
\operatorname{Re}\left\langle\tau_{V}^{\omega} \chi_{V}^{\nu}\right\rangle_{\boldsymbol{n}} q_{\nu}+\ldots \mathbf{O}_{-}=O\left(f^{\omega} g \boldsymbol{q}^{6} v_{\mathrm{F}}^{6} / \omega^{6}\right) \\
q \sim T, \quad \omega \sim 2 \Delta \\
\frac{1}{2 m^{*}} \psi^{\dagger}(\nabla-\boldsymbol{V})^{2} \psi \longrightarrow \\
\Gamma_{0}^{\omega, \xi}=f^{\omega, \xi} /\left(a^{2} \rho\left(n_{0}\right)\right)
\end{array}
$$

gauging of nucleon kinetic energy

\section{Neutrino emissivity}

Total emissivity $\varepsilon_{\nu \nu}=\varepsilon_{\nu \nu, V}+\varepsilon_{\nu \nu, A}$ sum of axial and vector current contributions

$$
\begin{gathered}
\varepsilon_{\nu \nu, a}=\frac{G^{2} g_{a}^{* 2}}{48 \pi^{4}} \int_{0}^{\infty} \mathrm{d} \omega \omega n_{\text {bos }}(\omega) \int_{0}^{\omega} \mathrm{d}|\boldsymbol{q}| \boldsymbol{q}^{2} \frac{\kappa_{a}}{a^{2}} \\
\kappa_{a}=\int \frac{\frac{d^{3} q_{1}}{2 \omega_{1}} \frac{d^{3} q_{2}}{2 \omega_{2}} \delta^{(4)}\left(q_{1}+q_{2}-q\right) \frac{3}{4 \pi} \operatorname{Im} \sum \chi_{a}(q)}{\text { leptonic phase space }}
\end{gathered}
$$


- neutrino emissivity on vector currents

$$
\begin{aligned}
& \kappa_{V}=\operatorname{Im}\left[\boldsymbol{q}^{2}\left\langle\chi_{V, 0}(\boldsymbol{n}, q)\right\rangle_{\boldsymbol{n}}+\left\langle\left(\boldsymbol{q} \boldsymbol{v}, \boldsymbol{q}_{\boldsymbol{\chi}}^{\chi_{V, 1}^{\prime}}(\boldsymbol{n}, q)\right\rangle_{\boldsymbol{n}}+\left(\omega^{2}-\underline{\boldsymbol{q}}_{-}^{2} \dagger\left\langle\boldsymbol{v} \boldsymbol{\chi}_{V, 1}(\boldsymbol{n}, q)\right\rangle_{\boldsymbol{n}}\right.\right.\right. \\
& \left.-\omega\left\langle(\boldsymbol{q} \boldsymbol{v}) \chi^{\prime}, 0,(\boldsymbol{n}, q)\right\rangle_{n}-\omega\left\langle\boldsymbol{q} \chi_{V, \mathbb{2}}(\boldsymbol{n}, \bar{q})\right\rangle_{\boldsymbol{n}}\right]
\end{aligned}
$$

in the limit $v_{F}<<1$ naively

$\longrightarrow$ we obtain the old result

$$
\begin{gathered}
\kappa_{V}=\boldsymbol{q}^{2} \operatorname{Im}\left\langle\chi_{V, 0}(\boldsymbol{n}, q)\right\rangle_{\boldsymbol{n}}=\boldsymbol{q}^{2} \operatorname{Im}\langle L(\boldsymbol{n}, q ;+1)\rangle_{\boldsymbol{n}} \simeq-\boldsymbol{q}^{2} a^{2} \rho \operatorname{Im} g\left(\omega^{2} / \Delta^{2}\right) \\
\bigsqcup_{\text {no vertex corrections ! }}
\end{gathered}
$$

$$
\epsilon_{\nu \nu}^{(0 n)}=\frac{4 \rho_{n} G^{2} \Delta_{n}^{7}}{15 \pi^{3}} I\left(\frac{\Delta_{n}}{T}\right) \quad I(z)=\int_{1}^{\infty} \frac{\mathrm{d} y y^{5}}{\sqrt{y^{2}-1}} e^{-2 z y}
$$

- neutrino emissivity on vector currents

$$
\begin{gathered}
\kappa_{V}=\operatorname{Im}\left[\boldsymbol{q}^{2}\left\langle\chi_{V, 0}(\boldsymbol{n}, q)\right\rangle_{\boldsymbol{n}}+\left\langle(\boldsymbol{q} \boldsymbol{v}) \boldsymbol{q} \chi_{V, 1}(\boldsymbol{n}, q)\right\rangle_{\boldsymbol{n}}+\left(\omega^{2}-\boldsymbol{q}^{2}\right)\left\langle\boldsymbol{v} \chi_{V, 1}(\boldsymbol{n}, q)\right\rangle_{\boldsymbol{n}}\right. \\
\left.-\omega\left\langle(\boldsymbol{q} \boldsymbol{v}) \chi_{V, 0}(\boldsymbol{n}, q)\right\rangle_{\boldsymbol{n}}-\omega\left\langle\boldsymbol{q} \chi_{V, 1}(\boldsymbol{n}, q)\right\rangle_{\boldsymbol{n}}\right]
\end{gathered}
$$

exploiting current conservation $\operatorname{Im}\left\langle\tau_{V}^{\omega} \chi_{V}^{\nu}\right\rangle_{n} q_{\nu}=0$

$$
\begin{aligned}
\kappa_{V} & =\frac{\left(\boldsymbol{q}^{2}-\omega^{2}\right) \operatorname{Im}\left\langle\chi_{V, 0}(\boldsymbol{n}, q)-\boldsymbol{v} \boldsymbol{\chi}_{V, 1}(\boldsymbol{n}, q)\right\rangle_{\boldsymbol{n}}>0}{\text { negative }} \\
\operatorname{Im}\left\langle\chi_{V, 0}(\boldsymbol{n}, q)\right\rangle & \approx-\frac{4 \boldsymbol{q}^{4} v_{\mathrm{F}}^{4}}{45 \omega^{4}} a^{2} \rho \operatorname{Im} g\left(\frac{\omega^{2}}{4 \Delta^{2}}\right)>0 \\
\operatorname{Im}\left\langle\boldsymbol{v} \chi_{V, 1}(\boldsymbol{n}, q)\right\rangle & \approx-\frac{2 \boldsymbol{q}^{2} v_{\mathrm{F}}^{4}}{9 \omega^{2}} a^{2} \rho \operatorname{Im} g\left(\frac{\omega^{2}}{4 \Delta^{2}}\right)>0 \\
\text { strongly suppressed } & \epsilon_{\nu, V}^{(n)} \simeq \frac{4}{81} v_{\mathrm{F}, \boldsymbol{n}}^{4} \epsilon_{\nu \nu}^{(0 n)}
\end{aligned}
$$


- neutrino emissivity on axial currents

$$
\begin{aligned}
\kappa_{A} & =\operatorname{Im}\left[\boldsymbol{q}^{2}\left\langle\boldsymbol{v} \chi_{A, 1}(\boldsymbol{n}, q)\right\rangle_{\boldsymbol{n}}+\left(3 \omega^{2}-2 \boldsymbol{q}^{2}\right)\left\langle\chi_{A, 0}(\boldsymbol{n}, q)\right\rangle_{\boldsymbol{n}}\right. \\
& \left.-\omega\left\langle\boldsymbol{q} \boldsymbol{\chi}_{A, 1}(\boldsymbol{n}, q)\right\rangle_{\boldsymbol{n}}-\omega\left\langle(\boldsymbol{q} \boldsymbol{v}) \chi_{A, 0}(\boldsymbol{n}, q)\right\rangle_{\boldsymbol{n}}\right]
\end{aligned}
$$

$$
\kappa_{A} \approx-a^{2} \rho v_{\mathrm{F}}^{2} \boldsymbol{q}^{2}\left[1+\left(1-\frac{2}{3} \frac{\boldsymbol{q}^{2}}{\omega^{2}}\right)-\frac{2}{3}\right] \operatorname{Im} g\left(\frac{\omega^{2}}{4 \Delta^{2}}\right)
$$

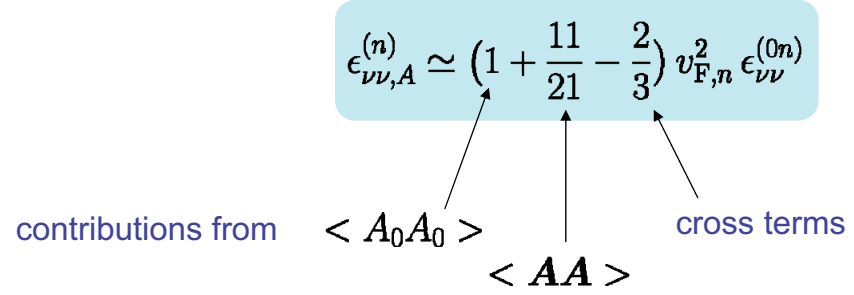

\section{Emissivity in pair formation breaking reactions}

$$
\begin{gathered}
R(\mathrm{nPFB})=\frac{\epsilon_{\nu \nu}^{\mathrm{nPBF}}}{\epsilon_{\nu \nu}^{(0 n)}} \simeq \frac{\epsilon_{\nu \nu, A}^{\mathrm{nPBF}}}{\epsilon_{\nu \nu}^{(0 n)}} \simeq \frac{6}{7} g_{A}^{* 2} v_{\mathrm{F}, n}^{2}=F_{n} v_{\mathrm{F}, n}^{2} . \\
n=n_{0}=0.17 \mathrm{fm}^{-3} \\
m^{*}=0.8 m
\end{gathered} \quad \begin{gathered}
F_{n} \simeq 0.9-1.2 \\
v_{\mathrm{F}, n} \simeq 0.36
\end{gathered} \quad R \simeq 0.12 \quad 0.15
$$

Main contribution is due to the axial current.

Suppression is of the order $\sim 0.1-0.3$ 
Conclusions ....

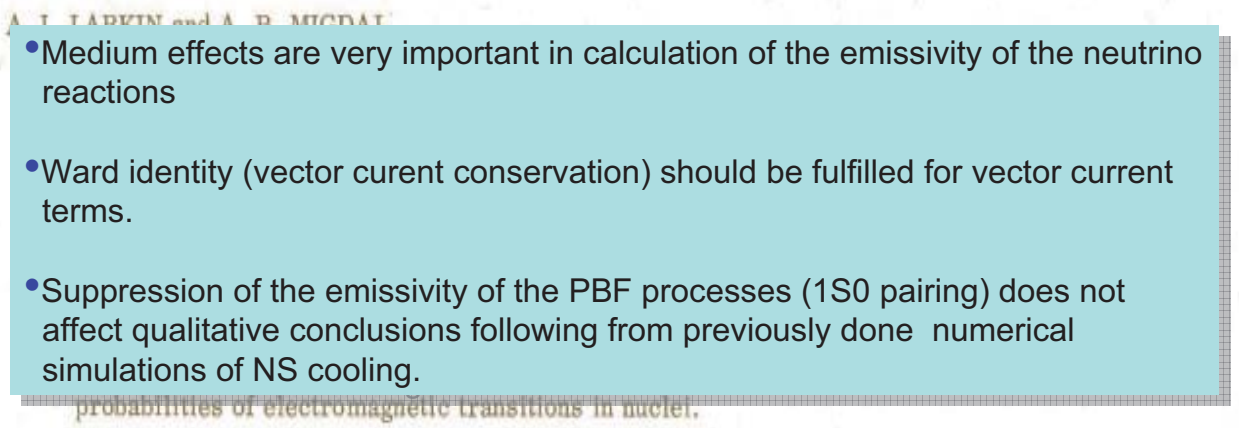

\section{INTRODUCTION}

IN

$I_{N}$ all real many-particle systems the interaction is not small, and therefore in the derivation of quantitative relations one cannot proceed, as is often done, by combining some part of the diagrams of perturbation theory. superconductors, the Debye temperature). Here one must introduce, in addition to $\Gamma^{\omega}$, one other function of the angles between the momenta of the quasi-particles, $\Gamma \xi$; the spherical harmonic of this function is connected with the width of the energy gap. It is natural to expect that the functions $\Gamma^{\omega}$ and $\Gamma^{\xi}$ depending on the angles between

\section{Nuclear medium cooling scenario}

Main ingredients

- Neutrino production process including in-medium effects Medium effects produce strong density dependence of the production rates

- Equation of state of nuclear matter

- Pairing gaps

- Nuclear equation of state Urbana-Argonne: $A 18+\delta v+U I X^{*}$ :

$$
M_{\text {crit }}^{\mathrm{DU}} \simeq 2 M_{\odot}, M_{\max } \simeq 2.2 M_{\odot}\left(n_{\text {cent }} \simeq 7 n_{0}\right) .
$$

but acausal for $n>4 n_{0}$.

Improvement: Heiselberg, Hjorth-Jensen (HHJ) causal interpolation EoS:

$$
M_{\text {crit }}^{\mathrm{DU}} \simeq 1.839 M_{\odot}, \quad M_{\text {max }} \simeq 1.96 M_{\odot}\left(n_{\text {cent }} \simeq 7 n_{0}\right)
$$


- Pairing gaps

The gap equation:

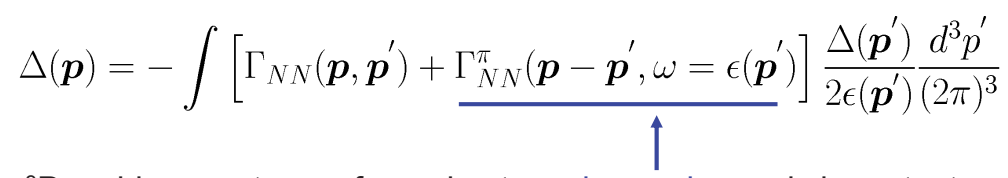

For ${ }^{3} \mathrm{P}_{2}$ pairing gap tensor force due to a pion exchange is important.

Model I

Numerical calculations by Schwenk and Friman [Phys. Rev. Lett., 92 (2004)], argued for a strong suppression of the ${ }^{3} \mathrm{P}_{2}$ neutron gaps, down to values $<10 \mathrm{keV}$, as the consequence of the medium-induced spin-orbit interaction.

\section{Model II}

Khodel et al. [Phys. Rev. Lett.,93 (2004)], assuming a proximity to a $2^{\text {nd }}$-order phase transition of pion condensation and a strong pion softening, estimated ${ }^{3} \mathrm{P}_{2}$ neutron pairing gap as large as $1-10 \sim \mathrm{MeV}$ in a broad region of densities.

Both possibilities checked within the "nuclear medium cooling scenario."

\section{Nuclear medium cooling scenario}

[Blaschke, Grigorian, Voskresensky, AA 424 (2004)]

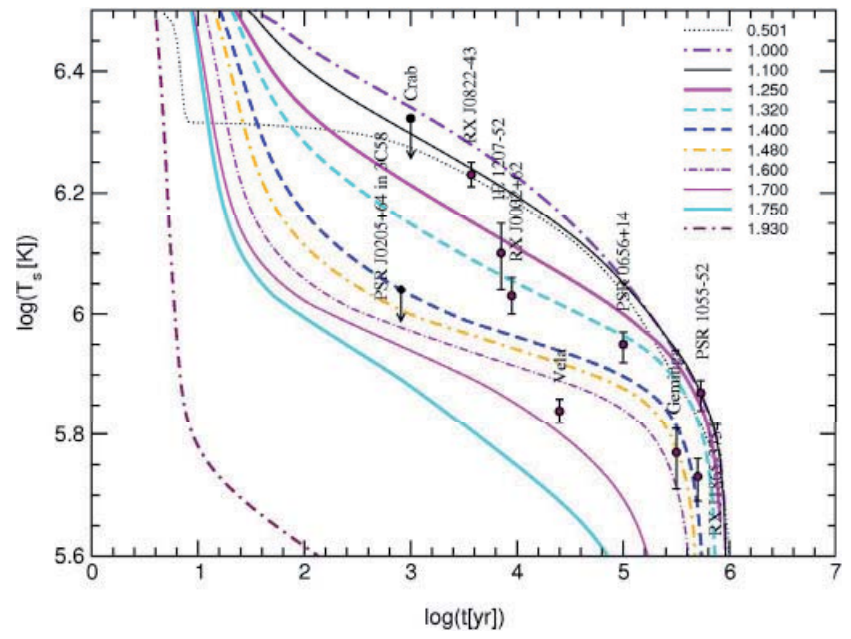

- ${ }^{3} P_{2}$ gaps from Schwenk \& Friman model.

- This result passed $\log N-\log S$ (population synthesis) controle 


\section{Nuclear medium cooling scenario}

[Grigorian, Voskresensky, A\&A (2005)]

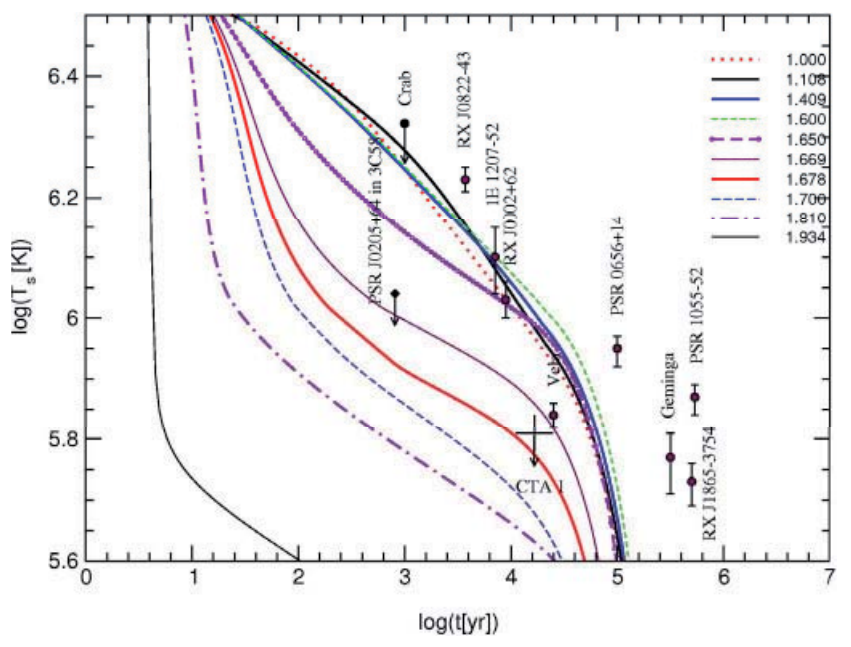

- ${ }^{3} P_{2}$ gaps from Khodel et al. model.

- No appropriate description of data.

\section{Conclusions}

In-medium effects have to be included otherwise calculations become inconsistent.

Large uncertainties remain due to poor knowledge of properties of dense matter

Comparison with data motivates strong density/(neutron star mass) dependence of medium effects and, thus, different neutron star masses in supernova remnants. 